\title{
14. APTIAN-ALBIAN FORAMINIFERS FROM SITE 766, CUVIER ABYSSAL PLAIN, AND COMPARISON WITH COEVAL FAUNAS FROM THE AUSTRALIAN REGION ${ }^{1}$
}

\author{
D. W. Haig ${ }^{2}$
}

\begin{abstract}
The distribution of 79 foraminiferal species is recorded among 23 samples taken from Cores 123-776A-16R through 123-766A-25R. The benthic species belong to the Ammodiscidae, Ammosphaeroidinidae, Spiroplectamminidae, Verneuilinidae, Tritaxiidae, Globotextulariidae, Eggerellidae, Ichthyolariidae, Nodosariidae, Vaginulinidae, Polymorphinidae, Glandulinidae, Bolivinidae, Turrilinidae, Fursenkoinidae, Pleurostomellidae, Patellinidae, Bagginidae, Quadrimorphinidae, Globorotalitidae, Osangulariidae, and Gavelinellidae families. The planktonic species belong to the Globigerinelloididae, Planomalinidae, Schackoinidae, Hedbergellidae, and Rotaliporidae families.

On the basis of foraminiferal biostratigraphy, the interval from Sections 123-766A-16R-3 through 123-766A-17R-3 has been placed confidently in the uppermost Albian (equivalent of the Rotalipora appenninica Zone of Caron, 1985). Below this, correlations are tentative because of the absence of commonly used zonal index species. The interval from lower Core 123-766A-17R to upper Core 123-766A-18R has been placed in the upper Albian; that from lower Core 123-766A-18R to Core 123-766A-21R has been included in the lower to middle Albian; and Cores 123-766A-24R and 123-766A-25R have been correlated with the upper Aptian.

The fauna is compared to that known from Aptian-Albian deposits in the Australian epeiric basins and at other sites along the western margin of the continent. These deposits were positioned at middle to high paleolatitudes in the Southern Hemisphere. The benthic assemblages from Site 766 belong to the Marssonella Association (of Haig, 1979) and are similar to those recorded elsewhere along the continental margin, but differ from those of the Ammobaculites Association (of Haig, 1979) within the epeiric basins. In overall aspect, the Australian fauna contains few genera not recorded elsewhere, but lacks many families present in Tethyan (low-latitude) faunas. Comparison of late Albian planktonic foraminifers known from along the continental margin indicates that assemblages from Site 766 are similar to those from the Papuan Basin at approximately the same paleolatitude, but are more diverse than those of the Naturaliste Plateau (approximately $10^{\circ}$ of paleolatitude south of Site 766), where Rotalipora and Planomalina are absent. This suggests that a significant gradient in surface water temperature existed on the late Albian continental margin between Site 766 and the Naturaliste Plateau.
\end{abstract}

\section{INTRODUCTION}

Aptian-Albian time witnessed one of the most extensive marine transgressions to cross the Australian continent during the Phanerozoic. The deposits resulting from this incursion occur in the great intracratonic basins that cover vast areas of Australia and extend north to the Papuan Basin of New Guinea and west over the North West Shelf and the Exmouth and Naturaliste submarine plateaus (Frakes et al., 1987; Fig. 1). According to Veevers (1984, p. 218), this transgression may have resulted from a general continental subsidence of the order of $150 \mathrm{~m}$, as well as a $105-\mathrm{m}$ eustatic rise in sea level. The Aptian-Albian deposits may be grouped as part of the Post-Gondwana Rift Megasequence recognized by Home et al. (1990) in the Papuan Basin. In most basins, fine-grained siliciclastic successions occur, dominated by carbonaceous mudstone, and the Aptian deposits include mudstone having a high radiolarian content (Ludbrook, 1978). Carbonate sediment, composed largely of the skeletons of pelagic microorganisms, is present only along the outer western margin of the continent and on the adjacent submarine plateaus and abyssal plains (Apthorpe, 1979).

An extensive knowledge of the foraminifers present in Australian Aptian-Albian deposits provides a basis for interpreting oceanographic conditions that existed in the region. The pioneering works of Crespin $(1944,1953,1963)$ were followed by com-

\footnotetext{
${ }^{1}$ Gradstein, F. M., Ludden, J. N., et al., 1992. Proc. ODP, Sci. Results, 123 College Station, TX (Ocean Drilling Program).

2 Department of Geology, University of Western Australia, Nedlands 6009 ,
}

prehensive documentation of the fauna of the "Great Artesian Basin” by Ludbrook (1966-Eromanga Basin), Scheibnerová (1971a, 1971b, 1972, 1974a, 1976, 1986-Eromanga Basin), Playford et al. (1975 - Carpentaria Basin), Haig and Barnbaum (1978 - Surat Basin), and Haig (1979a, 1980, 1982 - Surat, Eromanga, Carpentaria, and Laura basins). Part of the fauna of the Papuan Basin was described by Haig (1981) and Belford (1985) and further studies are being conducted by D. Lynch of the University of Western Australia. The fauna of the Western Australian marginal basins is less well documented. Herb (1974) and Scheibnerová (1978a) charted the distribution of species at Site 258 on the Naturaliste Plateau, and Quilty (1984) described several assemblages found among material dredged from Exmouth Plateau. The fauna from the onshore Carnarvon Basin is being studied by D. Haig, I. Copp, and G. Ellis of the University of Western Australia. Foraminifers from Aptian-Albian deposits on oceanic crust adjacent the western continental margin were recorded from the Perth Abyssal Plain (Scheibnerová, 1974b; Krasheninnikov, 1974a-DSDP Site 259), Cuvier Abyssal Plain (Scheibnerová, 1974b-DSDP Site 263), and the Gascoyne Abyssal Plain (Scheibnerová, 1974b; Krasheninnikov, 1974a-DSDP Site 260).

At Site 766 on the Cuvier Abyssal Plain (19 $55.985^{\prime} \mathrm{S}$; $110^{\circ} 27.130^{\prime}$ E; Fig. 1), a cored interval of Aptian-Albian nannofossil ooze and chalk was recovered (Ludden, Gradstein, et al., 1990; Fig. 2). This interval is almost $100 \mathrm{~m}$ thick, and although core recovery was poor in the lower half, the upper $50 \mathrm{~m}$ provides a comprehensive stratigraphic record. Most of the sediment is of pelagic origin, but may include material transported downslope from the plateau margin to the east, as some graded units occur. The foraminiferal record from Hole $766 \mathrm{~A}$ complements that from 


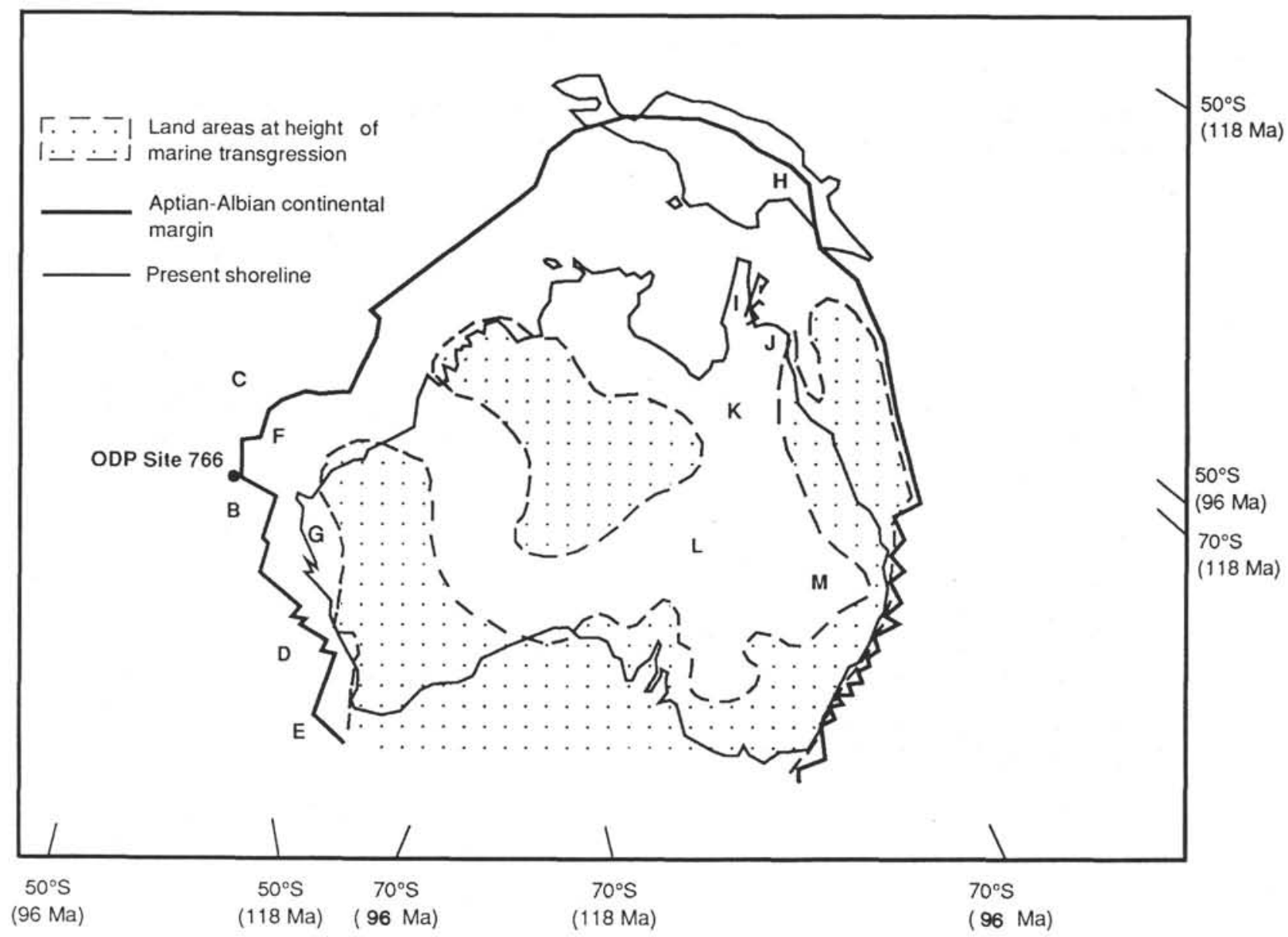

Figure 1. Reconstruction of Australian continent during the Aptian-Albian showing the locality of Site 766 and the maximum extent of the marine transgression across the continent (after Frakes et al., 1987). Paleolatitudes follow Veevers et al. (1991). Ogg et al. (this volume) suggest that paleolatitudes may be lower than shown here. Letters refer to the following localities recorded in Tables 5 through 9: A. Cuvier Abyssal Plain, Site 766, Cores 123-766A-16R through -25R (this study); B. Cuvier Abyssal Plain, DSDP Site 263, Cores 17 through 29 (Scheibnerová, 1974 b); C. Gascoyne Abyssal Plain, DSDP Site 260, Cores 9 through 18 (Scheibnerová, 1974b); D. Perth Abyssal Plain, DSDP Site 259, Cores 11 through 17 (Scheibnerová, 1974b); E. Naturaliste Plateau, DSDP Site 258, Cores 15 through 20 (Scheibnerová, 1978a); F. Exmouth Plateau dredge samples (Quilty, 1984); G. Eastern Carnarvon Basin (Haig et al., unpubl. data); H. Papuan Basin (Haig, 1981; Belford, 1985); I. Northern Carpentaria Basin (Haig, 1979a, 1980, 1982); J. Laura Basin (Haig, 1979a, 1980, 1982); K. Southern Carpentaria Basin (Playford et al., 1975; Haig, 1979a, 1980, 1982); L. Eromanga Basin (Crespin, 1963; Ludbrook, 1966; Scheibnerová, 1976; Haig, 1979a, 1980, 1982); M. Surat Basin (Haig and Barnbaum, 1978; Haig, 1979a, 1980, 1982).

DSDP Site 263 (Veevers, Heirtzler, et al., 1974; Scheibnerová, $1974 \mathrm{~b}$ ), approximately $300 \mathrm{~km}$ to the south, and adds substantially to our knowledge of the fauna of the western continental margin (Tables 1 through 9).

The aims of this study are (1) to document the composition of foraminiferal assemblages from Aptian-Albian deposits at Site 766 ; (2) to compare the fauna with that known elsewhere in oceanic deposits along the western continental margin and from Australian marginal and epeiric basins ; and (3) to place the regional fauna in a global biogeographic context.

\section{STRATIGRAPHIC DISTRIBUTION OF SPECIES AT SITE 766}

\section{Record of Species}

The study is based on 23 samples taken from Cores 123-766A$16 \mathrm{R}$ to $-25 \mathrm{R}$ in Hole $766 \mathrm{~A}$ (Fig. 2). Each sample comprised 15 $\mathrm{cm}^{3}$ of core material, which was disaggregated in boiling water and washed over a $63-\mu \mathrm{m}$ mesh. A comprehensive suite of foraminifers was recovered from each sand-fraction residue. The preservation of material is generally good, and only one sample (123-766A-18R-2, 83-85 cm) displays the effects of severe dis- solution. In this sample, only thick-shelled morphotypes have been preserved.

Species occurrences are charted in Tables 1 through 4 . The foraminifers are classified under Orders recognized by Haynes (1981), and Families and Genera diagnosed by Loeblich and Tappan (1988). Complete species nomenclature is referenced in the "taxonomic record" (Appendix) and is provided below only for taxa not recorded among the studied material.

\section{Order Astrorhizida}

Only species of the Ammodiscidae were located (Table 1), and representatives of this family are rare and sporadically distributed. The few recovered specimens suggest that a variable plexus of morphotypes is represented, similar to those illustrated from earlier Cretaceous deposits by Bartenstein (1974) and that the classification of these among various genera (following the scheme of Loeblich and Tappan, 1988) misrepresents the specific diversity.

\section{Order Lituolida}

Species of the Ammosphaeroidinidae, Spiroplectamminidae, Verneuilinidae, Tritaxiidae, Globotextulariidae, and Eggerellidae 


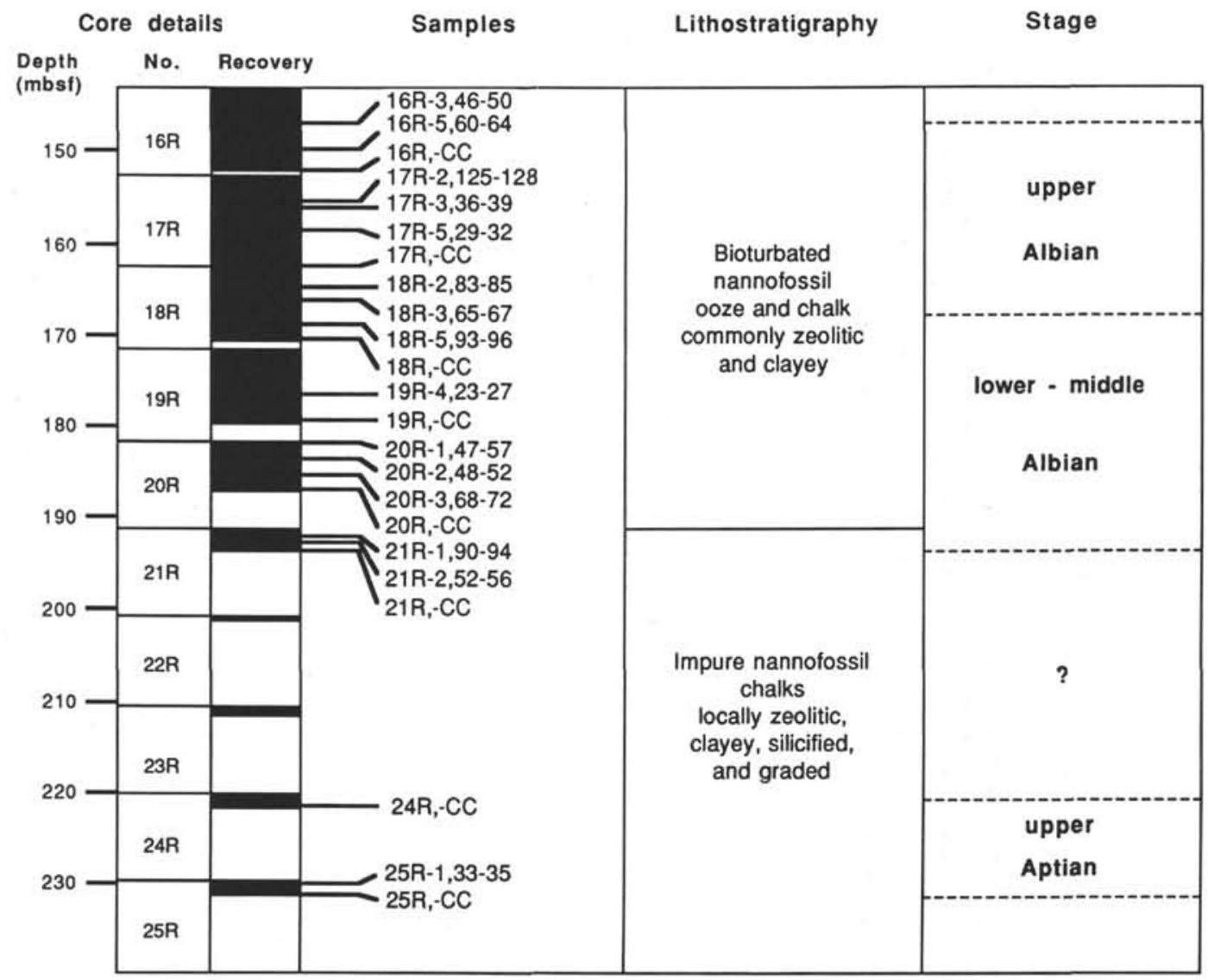

Figure 2. Positions of samples within Hole 766A, with lithostratigraphy and stage correlations.

families are recorded (Table 1). Some are common over a limited stratigraphic range, and several show morphoclinal interrelationships. All species are illustrated in Plates 1 and 2.

The Ammosphaeroidinidae family includes rare specimens attributed to Ammosphaeroidina that extend the range of this genus down to the mid-Cretaceous. Heterantyx cf. cretosa and Spiroplectinella cf. gandolfii are closely related species included in the Spiroplectamminidae; both are characterized by a prominent ridge between the adult chamber series, but differ in the nature of the test outline (Pl. 1, Figs. 5 through 7). Transitional forms between the species occur in Samples 123-766A-16R-5, $60-64 \mathrm{~cm}$, through $-17 \mathrm{R}-2,125-128 \mathrm{~cm}$, and suggest that Spiroplectinella arose from Heterantyx. The original record of $S$. gandolfii was from Cenomanian deposits in Morocco (Carbonnier, 1952), and other mid-Cretaceous occurrences of similar morphotypes include those of Spiroplectammina cretosa (Cushman) recorded by Moullade (1984) from the Atlantic Ocean and Spiroplectammina sp. described by Scheibnerova (1978b) from the South Atlantic.

The Verneuilinidae family includes species attributed to Gaudryina and Pseudogaudryinella. These are related to the Gaudryina-Spiroplectinata lineages outlined by Grabert (1959), but Spiroplectinata, with its compressed biserial stage and terminal aperture, is not represented here. The oldest assemblages contain specimens that have somewhat rectangular chamber margins in the adult stage and basal apertures (PI. 1, Figs. 8 and 9). These are compared to Gaudryina richteri, but have a narrower and less flaring biserial stage. Within Sample 123-766A-21R-CC, morphotypes appear that have the aperture extending areally above the basal suture of the last chamber. In some of the specimens, the final chamber has a uniserial stance, is cylindrical, and has a terminal aperture (Pl. 1, Fig. 11); these have been identified as Pseudogaudryinella sp. A. Higher in the succession, G. aff. richteri and Pseudogaudryinella sp. A have been progressively replaced by Pseudogaudryinella sp. B (Pl. 1, Fig. 12), which has a more robust test and rounded margins, and Pseudogaudryinella sp. C (Pl. 1, Figs. 13, 14) having a more narrowly elongate test and a more extensive biserial stage with inflated chambers. Although it is positioned separately in the Family Tritaxiidae, Tritaxia gaultina may be related to the Gaudryina-Pseudogaudryinella Group. Rare specimens from Sample 123-766A-21R-CC seem to be transitional between Pseudogaudryinella sp. A and $T$. gaultina. The development of an internal siphon within T. gaulti$n a$ has not been investigated here.

Intrachamber partitions are not clearly shown by the early forms attributed to Remesella sp. of the Globotextulariidae, but in Sample 123-766A-19R-4, 23-27 cm, and in higher assemblages, the partitions that partially subdivide the chamber interiors are distinct (Pl. 2, Fig. 2). This transition from simple to internally subdivided chambers is paralleled by that in the midCretaceous Arenobulimina-Voloshinoides Complex outlined by Barnard and Banner (1980). The buliminellid type of trochospiral coiling in the early stage of Remesella sp. bears some resemblance to the coiling mode of Arenobulimina; however, Remesella differs in its biserial adult stage (Pl. 2, Fig. 1) and broadly arched aperture.

The Eggerellidae family is represented by rare Dorothia sp. and Marssonella oxycona.

\section{Order Nodosariida}

Species of the Ichthyolariidae, Nodosariidae, Vaginulinidae, Polymorphinidae, and Glandulinidae families are recorded (Table 
Table 1. Distribution of species of Astrorhizida and Lituolida in Hole 766A (sample positions within the hole are shown in Fig. 2).

\begin{tabular}{|c|c|c|c|c|c|c|c|c|c|c|c|c|c|c|c|c|c|c|c|c|c|c|c|}
\hline $\begin{array}{r}\text { CORE } \\
\text { SECTION } \\
\text { INTERVAL (cm from top of section) }\end{array}$ & $\begin{array}{l}25 R \\
C C\end{array}$ & $\begin{array}{c}25 R \\
1 \\
33-35\end{array}$ & $\begin{array}{l}24 \mathrm{R} \\
\mathrm{CC}\end{array}$ & $\begin{array}{l}21 R \\
C C\end{array}$ & $\begin{array}{c}21 R \\
2 \\
52-56\end{array}$ & $\begin{array}{c}21 R \\
1 \\
90-94\end{array}$ & $\begin{array}{l}20 R \\
C C\end{array}$ & $\begin{array}{c}20 R \\
33 \\
68-72\end{array}$ & $\begin{array}{c}20 R \\
2 \\
48-52\end{array}$ & $\begin{array}{c}20 R \\
1 \\
47.57\end{array}$ & $\begin{array}{l}19 R \\
C C\end{array}$ & $\begin{array}{c}19 R \\
4 \\
23-27\end{array}$ & $\begin{array}{l}18 R \\
C C\end{array}$ & $\begin{array}{c}18 R \\
5 \\
93-96\end{array}$ & $\begin{array}{c}18 R \\
3 \\
65-67\end{array}$ & $\begin{array}{c}18 R \\
2 \\
83-85\end{array}$ & $\begin{array}{l}17 R \\
C C\end{array}$ & $\begin{array}{c}17 R \\
5 \\
29-32\end{array}$ & $\begin{array}{c}17 R \\
3 \\
36-39\end{array}$ & $\begin{array}{c}17 R \\
2 \\
125-128\end{array}$ & $\begin{array}{c}16 R \\
C C\end{array}$ & $\begin{array}{c}16 R \\
5 \\
60-64\end{array}$ & $\begin{array}{c}16 R \\
3 \\
46-50\end{array}$ \\
\hline \multicolumn{24}{|l|}{$\begin{array}{l}\text { ASTRORHIZIDA } \\
\text { Ammodiscidae }\end{array}$} \\
\hline ?Glomospirella sp. & $\ldots$ & $\ldots$ & $\ldots$ & $\ldots$ & $\ldots$ & $\ldots$ & & $\ldots$ & $\ldots$ & & R & & R & $\ldots$ & $\ldots$ & ... & R & ... & ... & $\ldots$ & R & $\ldots$ & $\ldots$ \\
\hline Repmania corona & $\cdots$ & $\cdots$ & $\cdots$ & $\cdots$ & $\cdots$ & $\cdots$ & R & $\cdots$ & $\cdots$ & R & $\cdots$ & R & $\ldots$ & $\cdots$ & $\cdots$ & $\cdots$ & R & $\cdots$ & $\ldots$ & $\ldots$ & R & $\cdots$ & $\cdots$ \\
\hline \multicolumn{24}{|l|}{$\begin{array}{l}\text { LITUOLIDA } \\
\text { Ammosphaeroidinidae }\end{array}$} \\
\hline $\begin{array}{l}\text { Ammosphaeroidina sp. } \\
\text { Spiroplectamminidae }\end{array}$ & $\ldots$ & $\ldots$ & $\ldots$ & ... & $\ldots$ & $\ldots$ & R & $\ldots$ & $\ldots$ & $R$ & $\mathrm{R}$ & $\ldots$ & $\ldots$ & $\ldots$ & $\ldots$ & $\ldots$ & $\ldots$ & $\mathrm{R}$ & $\ldots$ & $\ldots$ & c & $R$ & $\mathbf{R}$ \\
\hline Heterantyx cl. cretosa & $\ldots$ & $\ldots$ & $\ldots$ & $\ldots$ & $\ldots$ & $\ldots$ & $\ldots$ & $\ldots$ & $\ldots$ & $\ldots$ & $\ldots$ & $\ldots$ & $R$ & c & $R$ & ... & c & R & c & $\mathrm{R}$ & $R$ & $R$ & \\
\hline $\begin{array}{l}\text { Spiroplectinella ct. gandolfii } \\
\text { Verneuilinidae }\end{array}$ & $\ldots$ & $\ldots$ & $\ldots$ & $\ldots$ & $\ldots$ & $\ldots$ & $\ldots$ & ... & $\ldots$ & & $\ldots$ & $\ldots$ & $\ldots$ & $\ldots$ & $\ldots$ & $\cdots$ & ... & $\ldots$ & $\ldots$ & $\mathrm{R}$ & c & c & c \\
\hline $\begin{array}{l}\text { Gaudryina aft. richteri } \\
\text { Psoudogaudryinella sp. A }\end{array}$ & $\mathrm{R}$ & $\mathrm{R}$ & R & c & A & A & A & A & A & c & A & c & c & R & . & $\ldots$ & $\ldots$ & ... & $\cdots$ & $\ldots$ & $\ldots$ & $\ldots$ & $\ldots$ \\
\hline $\begin{array}{l}\text { Pseudogaudryinella sp. A } \\
\text { Pseudogaudryinella s. . B }\end{array}$ & $\ldots$ & $\ldots$ & $\ldots$ & c & $R$ & c & R & C & $R$ & R & $\begin{array}{l}\mathrm{C} \\
\mathrm{R}\end{array}$ & A & $\begin{array}{l}R \\
C\end{array}$ & $\begin{array}{l}R \\
C\end{array}$ & $\begin{array}{l}R \\
R\end{array}$ & $\dddot{R}$ & $\dddot{R}$ & $\dddot{R}$ & $\cdots$ & $\cdots$ & $\dddot{c}$ & $\dddot{c}$ & $\dddot{c}$ \\
\hline $\begin{array}{l}\text { Pseudogaudryinella sp. B } \\
\text { Pseudogaudryinella sp. C } \\
\text { Tritaxiidae }\end{array}$ & $\begin{array}{l}\cdots \\
\cdots\end{array}$ & $\begin{array}{l}\cdots \\
\cdots\end{array}$ & $\begin{array}{l}\cdots \\
\cdots\end{array}$ & $\begin{array}{l}\cdots \\
\ldots\end{array}$ & $\begin{array}{l}\cdots \\
\cdots\end{array}$ & $\begin{array}{l}\cdots \\
\cdots\end{array}$ & $\begin{array}{l}\cdots \\
\cdots\end{array}$ & $\begin{array}{l}\cdots \\
\cdots\end{array}$ & $\begin{array}{l}\cdots \\
\cdots\end{array}$ & $\begin{array}{l}\cdots \\
\cdots\end{array}$ & $\begin{array}{l}\mathrm{R} \\
\cdots\end{array}$ & $\ddot{R}$ & $\begin{array}{l}\text { C } \\
\text { R }\end{array}$ & A & $\begin{array}{l}\text { R } \\
C\end{array}$ & $\begin{array}{l}R \\
R\end{array}$ & R & $\begin{array}{l}H \\
C\end{array}$ & $\ddot{c}$ & $\dddot{c}$ & c & c & A \\
\hline $\begin{array}{l}\text { Tritaxia gaultina } \\
\text { Globotextulariidae }\end{array}$ & $\ldots$ & $\ldots$ & $\ldots$ & $?$ & ... & R & c & $\ldots$ & ... & $\ldots$ & R & $R$ & C & R & R & C & c & R & c & $\mathrm{R}$ & c & C & c \\
\hline $\begin{array}{l}\text { Remesella sp. } \\
\text { Eggerellidae }\end{array}$ & $\ldots$ & $\ldots$ & $\ldots$ & C & c & A & A & c & c & c & c & c & $R$ & c & R & R & C & c & c & c & c & c & A \\
\hline Dorothia sp. & $\ldots$ & $\ldots$ & $\ldots$ & $\mathrm{R}$ & R & $\mathrm{R}$ & R & ... & R & R & $\ldots$ & $\ldots$ & $\ldots$ & ... & ... & ... & ... & R & ... & $\ldots$ & R & R & $\ldots$ \\
\hline Marssoneilla oxycona & $\ldots$ & $\ldots$ & $\ldots$ & $\ldots$ & ... & ... & ... & ... & ... & $\ldots$ & $\ldots$ & $\ldots$ & $\ldots$ & ... & $\ldots$ & $\ldots$ & ... & ... & ... & $\ldots$ & $\ldots$ & R & $\ldots$ \\
\hline
\end{tabular}

Note: $\mathrm{A}=$ abundant; $\mathrm{C}=$ common; $\mathrm{R}=$ rare. 
Table 2. Distribution of species of Nodosariida in Hole 766A (sample positions within the hole are shown in Fig. 2).

\begin{tabular}{|c|c|c|c|c|c|c|c|c|c|c|c|c|c|c|c|c|c|c|c|c|c|c|c|}
\hline $\begin{array}{r}\text { CORE } \\
\text { SECTION } \\
\text { INTERVAL (cm from top of section) }\end{array}$ & $\begin{array}{l}25 R \\
\text { CC }\end{array}$ & $\begin{array}{c}25 R \\
1 \\
33-35\end{array}$ & $\begin{array}{l}24 R \\
\text { CC }\end{array}$ & $\begin{array}{l}21 R \\
C C\end{array}$ & $\begin{array}{c}21 R \\
2 \\
52 \cdot 56\end{array}$ & $\begin{array}{c}21 R \\
1 \\
90-94\end{array}$ & $\begin{array}{l}20 R \\
C C\end{array}$ & $\begin{array}{c}20 R \\
3 \\
68.72\end{array}$ & $\begin{array}{c}20 R \\
2 \\
48-52\end{array}$ & $\begin{array}{c}20 R \\
1 \\
47.57\end{array}$ & $\begin{array}{l}19 R \\
C C\end{array}$ & $\begin{array}{c}19 R \\
4 \\
23-27\end{array}$ & $\begin{array}{l}18 R \\
C C\end{array}$ & $\begin{array}{c}18 R \\
5 \\
93-96\end{array}$ & $\begin{array}{c}18 R \\
3 \\
65-67\end{array}$ & $\begin{array}{c}18 R \\
2 \\
83-85\end{array}$ & $\begin{array}{l}17 R \\
C C\end{array}$ & $\begin{array}{c}17 R \\
5 \\
29-32\end{array}$ & $\begin{array}{c}17 R \\
3 \\
36-39\end{array}$ & $\begin{array}{c}17 R \\
2 \\
125-128\end{array}$ & $\begin{array}{l}16 R \\
C C\end{array}$ & $\begin{array}{c}16 R \\
5 \\
60-64\end{array}$ & $\begin{array}{c}16 R \\
3 \\
46-50\end{array}$ \\
\hline \multicolumn{24}{|l|}{$\begin{array}{l}\text { Ichthyolariidae } \\
\text { Lingulonodosaria Ioryi }\end{array}$} \\
\hline $\begin{array}{l}\text { Lingulonodasaria loryi } \\
\text { Lingulonodasaria nodosaria }\end{array}$ & $\cdots$ & & $\mathrm{R}$ & $\mathrm{R}$ & & & $\mathrm{R}$ & c & R & $\ldots$ & P & $\begin{array}{l}R \\
R\end{array}$ & $\mathrm{R}$ & P & & ... & R & & ... & ... & $\cdots$ & $B$ & R \\
\hline $\begin{array}{l}\text { Lingulonodosaria nodosaria } \\
\text { Lingulonodosaria sp. A }\end{array}$ & $\begin{array}{l}\ldots \\
\ldots\end{array}$ & R & $\ldots$ & $\ldots$ & R & $\dddot{R}$ & $\ldots$ & $\mathrm{C}$ & P & $\ldots$ & $\ldots$ & $\ldots$ & $\ldots$ & H & P & $\ldots$. & $\begin{array}{l}\text { R } \\
\ldots\end{array}$ & $\begin{array}{l}\mathrm{R} \\
\ldots\end{array}$ & $\ldots$ & $\cdots$ & $\cdots$ & $\begin{array}{l}\mathrm{R} \\
\ldots\end{array}$ & $\begin{array}{l}\mathrm{B} \\
\ldots\end{array}$ \\
\hline $\begin{array}{l}\text { Lingulonodosaria sp. B } \\
\text { Nodosariidae }\end{array}$ & $\mathrm{R}$ & R & $\ldots$ & A & $\ldots$ & $\mathrm{R}$ & c & $\ldots$ & ... & $\ldots$ & $\ldots$ & $\ldots$ & $\ldots$ & $\ldots$ & $\ldots$ & $\ldots$ & $\ldots$ & $\ldots$ & .. & $\ldots$ & $\ldots$ & $\cdots$ & $\ldots$ \\
\hline ?Frondicularia sp. & $\ldots$ & $\ldots$ & ... & ... & $\ldots$ & $\ldots$ & 0 & $\ldots$ & $\ldots$ & $\ldots$ & $\ldots$ & $\ldots$ & $\ldots$ & $\ldots$ & $\ldots$ & $\ldots$ & ... & .. & ... & $\ldots$ & $\ldots$ & $\ldots$ & R \\
\hline ?Grigelis sp. & ... & ... & ... & \% & $\cdots$ & $\cdots$ & R & $\ldots$ & $\ldots$ & ... & ... & $\cdots$ & $\cdots$ & $\cdots$ & $\cdots$ & $\cdots$ & $\ldots$ & ... & $\cdots$ & $\cdots$ & $\ldots$ & $\ldots$ & $\cdots$ \\
\hline Laevidentalina cylindroides & $\cdots$ & $\cdots$ & $\cdots$ & R & ... & $\cdots$ & R & $\mathrm{R}$ & $\mathrm{R}$ & $\cdots$ & ... & ... & $\cdots$ & $\cdots$ & ... & $\cdots$ & $\mathrm{R}$ & ... & $\cdots$ & $\cdots$ & $\mathrm{R}$ & ... & $\cdots$ \\
\hline Laevidentalina gracilis & $\ldots$ & $\ldots$ & ... & A & $\ldots$ & $\ldots$ & \% & $\ldots$ & $\ldots$ & $\ldots$ & $\dddot{c}$ & $\dddot{c}$ & & $\dddot{R}$ & $\cdots$ & $\dddot{R}$ & $\dddot{R}$ & $\dddot{R}$ & $\dddot{R}$ & $\dddot{R}$ & $\dddot{a}$ & $\dddot{R}$ & $\begin{array}{l}\ldots . \\
\ldots .\end{array}$ \\
\hline Laevidentalina nana & $\cdots$ & R & $\ldots$ & $\mathrm{c}$ & $\mathrm{R}$ & $\mathrm{R}$ & $\begin{array}{c}C \\
B\end{array}$ & R & A & $\cdots$ & $\begin{array}{c}C \\
B\end{array}$ & $\begin{array}{c}\mathrm{B} \\
\mathrm{C}\end{array}$ & H & $\begin{array}{lll}\mathrm{H} \\
\mathrm{B}\end{array}$ & $\dddot{c}$ & $\mathrm{R}$ & & $\begin{array}{l}B \\
B\end{array}$ & & & a & R & $\ddot{R}$ \\
\hline Laevidentalina oligostegia & $\cdots$ & R & $\ldots$ & c & $\cdots$ & $\cdots$ & & $\dddot{0}$ & $\ddot{p}$ & $\cdots$ & R & $\mathrm{R}$ & & R & c & $\mathrm{R}$ & R & $\mathrm{R}$ & $\mathrm{R}$ & R & ... & R & R \\
\hline Lingulina buddencanyonensis & $\cdots$ & $\mathrm{R}$ & ... & $\ldots$ & $\dddot{n}$ & $\cdots$ & R & R & R & $\ldots$ & $\ldots$ & ... & ... & $\cdots$ & & ... & $\cdots$ & ... & $\cdots$ & $\cdots$ & ... & ... & $\cdots$ \\
\hline Lingulina sp. & & & & R & $\mathrm{R}$ & ... & $\cdots$ & $\mathrm{R}$ & ... & ... & ... & $\ldots$ & $\ldots$ & $\ldots$ & ... & ... & ... & ... & $\ldots$ & ... & $\ldots$ & & $\cdots$ \\
\hline Pseudonodosaria humilis & R & R & $\mathrm{R}$ & $\mathrm{R}$ & $\ldots$ & $\ldots$ & ... & ... & ... & $\ldots$ & $\ldots$ & R & $\ldots$ & R & $\ldots$ & ... & ... & ... & R & ... & R & R & $\cdots$ \\
\hline Pyramidulina obscura & R & R & .. & ... & ... & $\ldots$ & $\ldots$ & $\cdots$ & ... & ... & ... & ... & $\ldots$ & ... & $\ldots$ & ... & ... & ... & $\mathrm{R}$ & $\cdots$ & ... & ... & $\cdots$ \\
\hline Pyramidulina sceptrum & $\cdots$ & $\cdots$ & ... & ... & $\cdots$ & $\cdots$ & R & $\cdots$ & ... & $\ldots$ & ... & $\cdots$ & $\ldots$ & $\cdots$ & $\cdots$ & $\cdots$ & ... & $\cdots$ & $\cdots$ & ... & $\cdots$ & $\cdots$ & $\ldots$ \\
\hline Pyramidulina sp. & $\ldots$ & 8 & $\ldots$ & & & & & 8 & 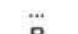 & $\cdots$ & 8 & 8 & ... & 0 & 8 & 8 & $\ldots$ & ... & $\ldots$ & 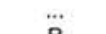 & 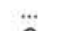 & 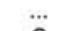 & R \\
\hline $\begin{array}{l}\text { Svenia cf. hamiltonensis } \\
\text { Vaginulinidae }\end{array}$ & R & R & $\ldots$ & $\mathrm{R}$ & R & $\mathrm{R}$ & c & P & R & $\cdots$ & $\mathrm{R}$ & R & $\ldots$ & R & $\mathrm{R}$ & $\mathrm{R}$ & R & $\ldots$ & $\mathrm{R}$ & R & c & c & c \\
\hline Astacolus ct. scitula & $\ldots$ & $\ldots$ & $\ldots$ & c & c & c & c & c & $\mathrm{R}$ & ... & $c$ & c & c & c & $\ldots$ & ... & $\ldots$ & $\ldots$ & & ... & ... & ... & $\ldots$ \\
\hline Astacolus sp. A & $\ldots$ & \% & $\ldots$ & $\ldots$ & ... & ... & $\cdots$ & ... & ... & $\ldots$ & $\mathrm{R}$ & $\cdots$ & $\cdots$ & $\mathrm{R}$ & \% & $\cdots$ & $\ldots$ & $\dddot{a}$ & $\mathrm{R}$ & $\cdots$ & $\cdots$ & .. & ... \\
\hline Astacolus spp. & $\mathrm{R}$ & c & R & $\ldots$ & ... & $\ldots$ & $\ldots$ & $\ldots$ & ... & $\mathbf{R}$ & $\ldots$ & $\ldots$ & ... & $\ldots$ & R & $\ldots$ & $\ldots$ & $\mathrm{R}$ & & $\ldots$ & $\ldots$ & $\cdots$ & ... \\
\hline Citharina cl. petila & $\ldots$ & R & $\mathrm{R}$ & $\ldots$ & $\ldots$ & R & $\mathrm{R}$ & R & ... & $\ldots$ & $\ldots$ & R & $\ldots$ & $\ldots$ & R & $\ldots$ & R & $\ldots$ & $\mathrm{R}$ & R & R & $\ldots$ & $\cdots$ \\
\hline Citharina sp. A & $\cdots$ & $\cdots$ & $\ldots$ & 0 & $\cdots$ & $\cdots$ & 0 & $\cdots$ & ... & $\cdots$ & ... & & $\ldots$ & $\dddot{p}$ & $\ldots$ & ... & $\ldots$ & ... & $\mathrm{R}$ & $\mathrm{R}$ & 8 & R & $\dddot{R}$ \\
\hline Citharina sp. B & $\ldots$ & ... & $\ldots$ & $\mathrm{R}$ & ... & $\cdots$ & R & ... & $\cdots$ & $\cdots$ & $\ldots$ & R & R & R & $\cdots$ & ... & ... & $\ldots$ & $\cdots$ & $\cdots$ & R & $\cdots$ & $\begin{array}{l}\text { R } \\
\ldots\end{array}$ \\
\hline $\begin{array}{l}\text { Hemirobulina hamulus } \\
\text { Lenticulina spp. }\end{array}$ & $\dddot{c}$ & $\dddot{A}$ & $\ddot{A}$ & $\ddot{c}$ & $\ddot{c}$ & $\dddot{c}$ & $\begin{array}{l}\mathrm{R} \\
\mathrm{C}\end{array}$ & $\ddot{A}$ & $\dddot{c}$ & $\dddot{R}$ & $\begin{array}{l}R \\
C\end{array}$ & $\dddot{c}$ & $\ddot{c}$ & $\begin{array}{l}R \\
R\end{array}$ & $\dddot{c}$ & $\dddot{c}$ & $\dddot{c}$ & $\begin{array}{l}R \\
R\end{array}$ & $\dddot{c}$ & $\dddot{R}$ & R & $\dddot{c}$ & $\dddot{c}$ \\
\hline 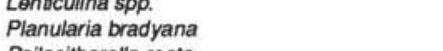 & $\ldots$ & $\ldots$ & $\ldots$ & $\ldots$ & $\ldots$ & $\ldots$ & $\ldots$ & $\ldots$ & ... & $\ldots$ & $R$ & $\ldots$ & $\ldots$ & $\ldots$ & $\ldots$ & $\ldots$ & $\ldots$ & $\ldots$ & $R$ & c & c & R & c \\
\hline Psilocitharella recta & $\ldots$ & $\dddot{m}$ & $\ldots$ & $\ldots$ & $\ldots$ & $\cdots$ & $\cdots$ & $\cdots$ & $\cdots$ & $\cdots$ & $\cdots$ & $\cdots$ & $\ldots$ & $\cdots$ & ... & ... & $\mathrm{R}$ & ... & $\cdots$ & $\cdots$ & ... & ... & $\cdots$ \\
\hline $\begin{array}{l}\text { Saracenaria spinosa } \\
\text { Saracenaria triangularis }\end{array}$ & R & R & c & $\cdots$ & $\cdots$ & $\cdots$ & ... & ... & $\cdots$ & $\cdots$ & $\ldots$ & ... & $\cdots$ & $\cdots$ & $\ldots$ & ... & ... & $\ldots$ & ... & ... & ... & ... & $\dddot{R}$ \\
\hline $\begin{array}{l}\text { Saracenaria triangularis } \\
\text { Vaginutina so. }\end{array}$ & $\cdots$ & $\ddot{R}$ & $\cdots$ & $\cdots$ & $\cdots$ & $\cdots$ & $\ddot{\theta}$ & $\dddot{q}$ & $\cdots$ & $\cdots$ & $\cdots$ & $\cdots$ & $\cdots$ & $\cdots$ & R & $\cdots$ & $\dddot{8}$ & $\mathrm{R}$ & $\dddot{8}$ & $\dddot{R}$ & $\dddot{c}$ & $\dddot{8}$ & $\begin{array}{l}R \\
R\end{array}$ \\
\hline $\begin{array}{l}\text { Vaginulina sp. } \\
\text { Vaginulinopsis sp. }\end{array}$ & … & R & $\cdots$ & $\dddot{\mathrm{R}}$ & $\cdots$ & $\begin{array}{l}\cdots \\
\ldots\end{array}$ & $\begin{array}{l}\mathrm{H} \\
\ldots\end{array}$ & $\begin{array}{l}R \\
R\end{array}$ & $\cdots$ & $\cdots$ & $\cdots$ & $\cdots$ & $\cdots$ & $\cdots$ & $\ldots$ & $\cdots$ & $\mathrm{R}$ & … & R & A & c & $\mathrm{R}$ & $\begin{array}{l}\mathrm{R} \\
\ldots\end{array}$ \\
\hline $\begin{array}{l}\text { Vaginulinopsis } s p \text {. } \\
\text { Polymorphinidae }\end{array}$ & & $\cdots$ & $\cdots$ & & $\cdots$ & $\cdots$ & -.. & $\mathrm{H}$ & $\cdots$ & $\cdots$ & $\cdots$ & $\cdots$ & $\cdots$ & "' & $\cdots$ & -.. & ‥ & ... & -.. & $\cdots$ & -. & $\cdots$ & ${ }^{B}$ \\
\hline Globulina prisca & $\ldots$ & $\cdots$ & $\ldots$ & R & $\ldots$ & $\ldots$ & R & $\ldots$ & $\ldots$ & $\ldots$ & $\ldots$ & $\ldots$ & $\ldots$ & ... & $\ldots$ & $\ldots$ & $\mathrm{R}$ & $\ldots$ & $\ldots$ & $\ldots$ & $\ldots$ & $\ldots$ & $\begin{array}{l}R \\
R\end{array}$ \\
\hline $\begin{array}{l}\text { Ramulina spp. } \\
\text { Glandulinidao }\end{array}$ & $\cdots$ & $\cdots$ & A & $\mathrm{R}$ & $\cdots$ & ... & c & ... & $\cdots$ & ... & R & ... & $\cdots$ & $\cdots$ & $\cdots$ & $\cdots$ & R & ... & $\mathrm{R}$ & $\cdots$ & $\mathrm{R}$ & $\cdots$ & $\mathbf{R}$ \\
\hline Tricarinella excavata & $\cdots$ & $\cdots$ & $\cdots$ & $\cdots$ & $\cdots$ & $\ldots$ & R & $\ldots$ & $\ldots$ & $\ldots$ & $\ldots$ & R & $\ldots$ & $\ldots$ & $\ldots$ & $\ldots$ & $\mathrm{R}$ & $\cdots$ & $\cdots$ & $\cdots$ & $\cdots$ & $\cdots$ & ... \\
\hline
\end{tabular}


Table 3. Distribution of species of Buliminida and Rotaliida in Hole 766A (sample positions within the hole are shown in Fig. 2).

\begin{tabular}{|c|c|c|c|c|c|c|c|c|c|c|c|c|c|c|c|c|c|c|c|c|c|c|c|}
\hline $\begin{array}{r}\text { CORE } \\
\text { SECTION } \\
\text { INTERVAL (cm from top of section) }\end{array}$ & $\begin{array}{l}25 R \\
C C\end{array}$ & $\begin{array}{c}25 R \\
1 \\
33-35\end{array}$ & $\begin{array}{l}24 R \\
C C\end{array}$ & $\begin{array}{l}21 R \\
C C\end{array}$ & $\begin{array}{c}21 R \\
2 \\
52 \cdot 56\end{array}$ & $\begin{array}{c}21 R \\
1 \\
90-94\end{array}$ & $\begin{array}{l}20 R \\
C C\end{array}$ & $\begin{array}{c}20 R \\
3 \\
68-72\end{array}$ & $\begin{array}{c}20 R \\
2 \\
48-52\end{array}$ & $\begin{array}{c}20 R \\
1 \\
47.57\end{array}$ & $\begin{array}{l}19 R \\
C C\end{array}$ & $\begin{array}{c}19 R \\
4 \\
23-27\end{array}$ & $\begin{array}{l}18 R \\
C C\end{array}$ & $\begin{array}{c}18 R \\
5 \\
93-96\end{array}$ & $\begin{array}{c}18 R \\
3 \\
65-67\end{array}$ & $\begin{array}{c}18 R \\
2 \\
83-85\end{array}$ & $\begin{array}{l}17 R \\
C C\end{array}$ & $\begin{array}{c}17 R \\
5 \\
29 \cdot 32\end{array}$ & $\begin{array}{c}17 R \\
3 \\
36-39\end{array}$ & $\begin{array}{c}17 R \\
2 \\
125 \cdot 128\end{array}$ & $\begin{array}{l}16 R \\
C C\end{array}$ & $\begin{array}{c}16 R \\
5 \\
60-64\end{array}$ & $\begin{array}{c}16 R \\
3 \\
46-50\end{array}$ \\
\hline \multicolumn{24}{|l|}{$\begin{array}{l}\text { BULIMINIDA } \\
\text { Bolivinidae }\end{array}$} \\
\hline $\begin{array}{l}\text { Tappanina sp. } \\
\text { Turrilinidae }\end{array}$ & $\ldots$ & $\ldots$ & $\ldots$ & $\ldots$ & ... & $\cdots$ & $\cdots$ & $\ldots$ & $\ldots$ & $\cdots$ & $\cdots$ & $\ldots$ & $\cdots$ & $\ldots$ & $\ldots$ & $\cdots$ & $\ldots$ & $\ldots$ & $\cdots$ & $\cdots$ & $\cdots$ & A & c \\
\hline Cuneus ct. ludbrookae & & & $\ldots$ & & & & & . & & $\cdots$ & & & & & 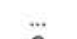 & $\ldots$ & $\ldots$ & & & $\ldots$ & $\ldots$ & R & $\mathrm{R}$ \\
\hline ?Eurycheilostoma ct. hergottensis & $\mathrm{R}$ & c & $\ldots$ & R & c & R & R & c & R & $\ldots$ & R & R & $\mathrm{R}$ & c & c & A & $\ldots$ & c & A & $\ldots$ & $\ldots$ & R & R \\
\hline $\begin{array}{l}\text { Praebulimina ct. nannina } \\
\text { Fursenkoinidae }\end{array}$ & $R$ & $\ldots$ & $\ldots$ & R & $\mathrm{R}$ & A & $\mathrm{R}$ & A & c & $\mathrm{R}$ & c & c & $\mathrm{R}$ & $\ldots$ & R & $\ldots$ & $\mathrm{R}$ & $\ldots$ & ... & ... & $\ldots$ & $\cdots$ & $\ldots$ \\
\hline $\begin{array}{l}\text { Coryphostoma sp. } \\
\text { Pleurostomellidae }\end{array}$ & c & $\mathrm{R}$ & c & A & c & c & c & A & c & $\mathrm{R}$ & c & A & c & c & R & $\ldots$ & A & c & $\mathrm{R}$ & R & c & R & c \\
\hline Ellipsoglandulina sp. & $\ldots$ & ... & $\ldots$ & $\ldots$ & .... & $\ldots$ & $\ldots$ & $\ldots$ & & $\ldots$ & $\ldots$ & $\ldots$ & $\ldots$ & & $\ldots$ & $\ldots$ & 0 & ... & ... & $\ldots$ & R & $\mathrm{R}$ & ... \\
\hline Ellipsoidella ct. cuneata & $\ldots$ & $\cdots$ & $\cdots$ & $\ddot{c}$ & $\ddot{c}$ & R & c & c & & $\cdots$ & $\ldots$ & $\dddot{p}$ & $\ldots$ & R & $\dddot{0}$ & $\ldots$ & $\mathrm{R}$ & $\ldots$ & $\ddot{c}$ & $\dddot{p}$ & R & $\ldots$ & $\ldots$ \\
\hline Pleurostomella aff. reussi & $\ldots$ & ... & ... & c & c & c & c & c & R & $\ldots$ & A & R & $\ldots$ & P & A & c & C & A & C & A & C & c & A \\
\hline \multicolumn{24}{|l|}{$\begin{array}{l}\text { ROTALIIDA } \\
\text { Patellinidae }\end{array}$} \\
\hline $\begin{array}{l}\text { Hergottella sp. } \\
\text { Bagginidae }\end{array}$ & $\ldots$ & c & $\mathrm{R}$ & & ... & $\ldots$ & $\ldots$ & $\ldots$ & ... & $\ldots$ & $\cdots$ & $\ldots$ & $\cdots$ & $\cdots$ & $\ldots$ & $\ldots$ & $\cdots$ & ... & $\cdots$ & $\ldots$ & $\ldots$ & ... & ... \\
\hline Serovaina gracillima & $\ldots$ & $\ldots$ & $\ldots$ & $\mathrm{R}$ & $\cdots$ & $\ldots$ & R & R & R & $\cdots$ & R & c & C & c & c & & A & $\mathrm{R}$ & R & c & c & R & c \\
\hline $\begin{array}{l}\text { Serovaina infracretacea } \\
\text { Quadrimorphinidae }\end{array}$ & A & A & A & A & A & A & A & A & A & A & A & A & c & A & A & R & A & c & R & c & c & c & A \\
\hline $\begin{array}{l}\text { Quadrimorphina allomorphinoides } \\
\text { Globorotalitidae }\end{array}$ & $\cdots$ & $\cdots$ & $\ldots$ & $\ldots$ & $\ldots$ & $\ldots$ & $\mathrm{R}$ & R & $\ldots$ & ... & $\ldots$ & ... & $\ldots$ & $\ldots$ & $\ldots$ & $\ldots$ & c & $\mathrm{R}$ & $\mathrm{R}$ & $\mathrm{R}$ & A & $\ldots$ & R \\
\hline $\begin{array}{l}\text { Globorotalites aptiensis } \\
\text { Osangulariidae }\end{array}$ & $\cdots$ & $\ldots$ & $\ldots$ & $\ldots$ & $\ldots$ & $\ldots$ & $\ldots$ & $\ldots$ & ... & $\ldots$ & $\cdots$ & $\ldots$ & $\cdots$ & $\cdots$ & $\ldots$ & $\ldots$ & $\ldots$ & ... & ... & $\ldots$ & $\ldots$ & c & $\ldots$ \\
\hline Chartionia cf. australis & $\ldots$ & ... & $\ldots$ & $\ldots$ & $\ldots$ & $\ldots$ & c & c & R & $\ldots$ & $\cdots$ & R & & & $\ldots$ & & c & $\ldots$ & R & & c & R & ... \\
\hline $\begin{array}{l}\text { Osangularia schloenbachi } \\
\text { Gavelinellidae }\end{array}$ & $\cdots$ & $\ldots$ & $\ldots$ & A & A & A & A & A & A & A & A & A & c & A & A & $\mathrm{R}$ & A & A & c & R & c & c & A \\
\hline Berthelina intermedia & $\cdots$ & & & R & A & A & A & A & c & c & $\mathrm{R}$ & c & R & R & R & R & A & c & c & c & R & c & $\mathrm{R}$ \\
\hline Gavelinella ct. andersoni & A & A & A & c & A & c & c & $R$ & $\ldots$ & $\mathrm{R}$ & $\ldots$ & $\ldots$ & c & A & c & c & A & c & c & ... & R & c & A \\
\hline Gavelinella ct. indica & $\ldots$ & ... & $\ldots$ & $\ldots$ & $\ddot{a}$ & $\ldots$ & $\ldots$ & A & R & $\ldots$ & $\ldots$ & ... & $\cdots$ & $\cdots$ & $\cdots$ & ... & $\cdots$ & ... & ... & ... & $\ldots$ & ... & $\ldots$ \\
\hline Gavelinella sp. A & $\ldots$ & $\ldots$ & c & c & c & A & A & c & c & c & $\mathrm{R}$ & ... & $\cdots$ & $\ldots$ & .. & ... & ... & ... & ... & $\ldots$ & $\ldots$ & $\ldots$ & .. \\
\hline Gavelinella sp. B & C & A & R & c & c & c & C & $\cdots$ & $\cdots$ & c & $\cdots$ & R & $\ddot{A}$ & $\ddot{c}$ & $\dddot{c}$ & $\dddot{R}$ & $\dddot{A}$ & $\ddot{A}$ & $\dddot{c}$ & $\dddot{c}$ & $\dddot{c}$ & $\dddot{c}$ & $\ddot{A}$ \\
\hline Scheibnerova protindica & $\cdots$ & $\cdots$ & $" \cdots$ & $\cdots$ & $\cdots$ & $\cdots$ & $\cdots$ & $\cdots$ & & $\cdots$ & $\cdots$ & & $n$ & & & & & & & & & & \\
\hline
\end{tabular}

Note: $\mathrm{A}=$ abundant $\mathrm{C}=$ common; $\mathrm{R}=$ rare, 
Table 4. Distribution of species of Globigerinida in Hole 766A (sample positions within the hole are shown in Fig. 2).

\begin{tabular}{|c|c|c|c|c|c|c|c|c|c|c|c|c|c|c|c|c|c|c|c|c|c|c|c|}
\hline $\begin{array}{r}\text { CORE } \\
\text { SECTION } \\
\text { INTERVAL (cm from top of section) }\end{array}$ & $\begin{array}{l}25 \mathrm{R} \\
\mathrm{CC}\end{array}$ & $\begin{array}{c}25 R \\
1 \\
33-35\end{array}$ & $\begin{array}{l}24 \mathrm{R} \\
\mathrm{CC}\end{array}$ & $\begin{array}{l}21 \mathrm{R} \\
\mathrm{CC}\end{array}$ & $\begin{array}{c}21 R \\
2 \\
52-56\end{array}$ & $\begin{array}{c}21 R \\
1 \\
90-94\end{array}$ & $\begin{array}{l}20 \mathrm{R} \\
\mathrm{CC}\end{array}$ & $\begin{array}{c}20 \mathrm{P} \\
3 \\
68.72\end{array}$ & $\begin{array}{c}20 R \\
2 \\
48-52\end{array}$ & $\begin{array}{c}20 R \\
1 \\
47.57\end{array}$ & $\begin{array}{l}19 R \\
\text { CC }\end{array}$ & $\begin{array}{c}19 R \\
4 \\
23-27\end{array}$ & $\begin{array}{l}18 \mathrm{R} \\
\mathrm{CC}\end{array}$ & $\begin{array}{c}18 \mathrm{R} \\
5 \\
93-96\end{array}$ & $\begin{array}{c}18 R \\
3 \\
65-67\end{array}$ & $\begin{array}{c}18 \mathrm{R} \\
2 \\
83-85\end{array}$ & $\begin{array}{l}17 \mathrm{R} \\
\mathrm{CC}\end{array}$ & $\begin{array}{c}17 R \\
5 \\
29-32\end{array}$ & $\begin{array}{c}17 \mathrm{R} \\
3 \\
36-39\end{array}$ & $\begin{array}{c}17 \text { A } \\
2 \\
125-128\end{array}$ & $\begin{array}{l}16 \mathrm{R} \\
C \mathrm{C}\end{array}$ & $\begin{array}{c}16 R \\
5 \\
60-64\end{array}$ & $\begin{array}{c}16 R \\
3 \\
46-50\end{array}$ \\
\hline \multicolumn{24}{|l|}{ Globigerinelloididae } \\
\hline $\begin{array}{l}\text { Globigerinelloidos aptiense } \\
\text { Globigerinelloides ct. aptiense }\end{array}$ & $\begin{array}{l}A \\
C\end{array}$ & R & $\ldots$ & $\ldots$ & $\ldots$ & ... & ... & $\ldots$ & $\ldots$ & $\ldots$ & $\ldots$ & $\cdots$ & $\ldots$ & $\ldots$ & $\ldots$ & $\ldots$ & $\ldots$ & $\ldots$ & $\ldots$ & $\ldots$ & $\ldots$ & $\ldots$ & $\ldots$ \\
\hline $\begin{array}{l}\text { Globigerinelloides ct. bentonensis } \\
\text { Planomalinidae }\end{array}$ & $c$ & $\mathrm{R}$ & c & $\ldots$ & ... & $\ldots$ & $\ldots$ & ... & R & R & $\ldots$ & $\ldots$ & $\ldots$ & $\ldots$ & $\ldots$ & $\ldots$ & $\ldots$ & $\ldots$ & $\ldots$ & ... & $\ldots$ & $\ldots$ & $\ldots$ \\
\hline Planomalina praebuxtorti & $\ldots$ & $\ldots$ & $\ldots$ & $\ldots$ & $\ldots$ & ... & $\ldots$ & ... & $\ldots$ & $\ldots$ & $\ldots$ & $\ldots$ & $\ldots$ & ... & $\ldots$ & $\ldots$ & ... & $\ldots$ & c & c & $\ldots$ & $\cdots$ & $\cdots$ \\
\hline $\begin{array}{l}\text { Planomalina buxtorfi } \\
\text { Schackoinidae }\end{array}$ & $\ldots$ & ... & $\ldots$ & $\ldots$ & $\ldots$ & ... & $\ldots$ & ... & ... & ... & ... & $\ldots$ & $\ldots$ & ... & $\ldots$ & ... & $\ldots$ & $\ldots$ & ... & $\ldots$ & c & c & A \\
\hline $\begin{array}{l}\text { Schackoina cenomana } \\
\text { Hedbergellidae }\end{array}$ & ... & $\ldots$ & $\ldots$ & $\ldots$ & $\ldots$ & $\ldots$ & ... & $\ldots$ & ... & $\ldots$ & $\ldots$ & ... & $\ldots$ & ... & $\ldots$ & $\ldots$ & $\ldots$ & ... & ... & ... & $\mathrm{R}$ & $\ldots$ & c \\
\hline Clavihedbergella simplicissima & $\ldots$ & $\ldots$ & ... & ... & $\ldots$ & $\ldots$ & ... & $\ldots$ & & $\ldots$ & & $\ldots$ & & $\ldots$ & & $\ldots$ & & R & R & & c & c & $\ldots$ \\
\hline Clavihedbergella sp. & $\ldots$ & $\ldots$ & $\ldots$ & $\ldots$ & $\ldots$ & $\ldots$ & A & A & $\mathrm{R}$ & $\ldots$ & $\mathrm{R}$ & $\ldots$ & c & $\ldots$ & $\mathrm{R}$ & $\ldots$ & c & $\mathrm{R}$ & $\mathrm{R}$ & c & $\ldots$ & $\ldots$ & $\ldots$ \\
\hline Hedbergella sp. ct. Blefuscuiana aptica & A & A & c & $\ldots$ & $\ldots$ & R & $\ldots$ & R & $\ldots$ & $\mathrm{R}$ & $\ldots$ & $\ldots$ & $\ldots$ & $\ldots$ & $\ldots$ & $\ldots$ & $\ldots$ & $\ldots$ & $\ldots$ & $\ldots$ & $\ldots$ & $\ldots$ & ... \\
\hline Hedbergella ct. planispira & $\ldots$ & A & A & A & A & A & A & A & R & R & $\ldots$ & $\ldots$ & $\ldots$ & $\ldots$ & $\ldots$ & $\ldots$ & ... & $\ldots$ & $\ldots$ & ... & $\cdots$ & $\cdots$ & $\cdots$ \\
\hline Hedbergella planispira & $\ldots$ & $\ldots$ & $\ldots$ & ... & $\cdots$ & $\ldots$ & $\ldots$ & $\ldots$ & A & A & A & A & $A$ & A & $c$ & & 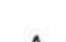 & 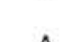 & 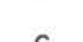 & 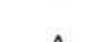 & & & A \\
\hline $\begin{array}{l}\text { Hedbergella ct. punctata } \\
\text { Hedbergella trocoidea }\end{array}$ & $\cdots$ & ?R & $\cdots$ & $\ddot{c}$ & $\cdots$ & ?R & $\dddot{c}$ & $\cdots$ & $\cdots$ & $\dddot{R}$ & $\cdots$ & R & R & R & c & $\cdots$ & A & A & C & A & C & A & A \\
\hline Hedbergella yezoana & $\ldots$ & $\ldots$ & $\ldots$ & ... & $\ldots$ & $\ldots$ & $\ldots$ & $\ldots$ & $\ldots$ & ... & c & c & A & A & A & R & A & A & $c$ & A & $\ldots$ & $\ldots$ & A \\
\hline $\begin{array}{l}\text { Praeglobotruncana delrioensis } \\
\text { Rotaliporidae }\end{array}$ & $\cdots$ & 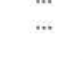 & $\ldots$ & $\ldots$ & $\cdots$ & $\ldots$ & $\ldots$ & $\ldots$ & ... & $\cdots$ & ... & $\ldots$ & $\ldots$ & $\ldots$ & $\ldots$ & $\ldots$ & $\ldots$ & $\ldots$ & $\ldots$ & $\ldots$ & $\ddot{A}$ & R & A \\
\hline Rotalipora appenninica & $\ldots$ & ... & $\ldots$ & $\ldots$ & $\ldots$ & ... & $\ldots$ & ... & ... & $\ldots$ & ... & $\ldots$ & $\ldots$ & $\ldots$ & $\ldots$ & ... & $\ldots$ & ... & $\ldots$ & $\ldots$ & $\ldots$ & R & A \\
\hline Rotalipora ticinensis & $\ldots$ & ... & $\ldots$ & ... & ... & ... & $\ldots$ & ... & ... & $\ldots$ & $\ldots$ & $\ldots$ & $\ldots$ & $\ldots$ & $\ldots$ & $\ldots$ & $\ldots$ & $\ldots$ & $\ldots$ & $\ldots$ & $\ldots$ & $\mathrm{R}$ & A \\
\hline
\end{tabular}

Note: $\mathrm{A}=$ abundant; $\mathrm{C}=$ common; $\mathrm{R}=$ rare. 
Table 5. Distribution of foraminiferal genera of Astrorhizida in confirmed Aptian-Albian deposits of the western margin submarine plateaus and abyssal plains and the Australian epeiric basins.

\begin{tabular}{|c|c|c|c|c|c|c|c|c|c|c|c|c|c|}
\hline & \multicolumn{6}{|c|}{ WESTERN MARGIN } & \multicolumn{7}{|c|}{ EPEIRIC BASINS } \\
\hline & A & B & C & D & $\mathbf{E}$ & $\mathbf{F}$ & G & H & 1 & $J$ & $\mathbf{K}$ & $\mathbf{L}$ & M \\
\hline Bathysiphonidae & & & & & & & & & & & & & \\
\hline $\begin{array}{l}\text { Bathysiphon } \\
\text { Silicotubidae }\end{array}$ & .. & $x$ & $?$ & & $?$ & .. & .. & $x$ & $x$ & $x$ & .. & $x$ & .. \\
\hline $\begin{array}{l}\text { Silicotuba } \\
\text { Psammosphaeridae }\end{array}$ & .. & .. & .. & .. &.. & .. & .. & .. & $x$ & $x$ & $x$ & $x$ & $x$ \\
\hline $\begin{array}{l}\text { Psammosphaera } \\
\text { Saccamminidae }\end{array}$ & .. & .. & .. & .. & .. & $?$ & .. & $x$ & $x$ & $x$ & $x$ & $x$ & $x$ \\
\hline Lagenammina & .. & ... & $?$ &.. & .. & .. & .. & .. & .. & $x$ & $x$ & $x$ & $x$ \\
\hline $\begin{array}{l}\text { Placentammina } \\
\text { Hippocrepinidae }\end{array}$ & .. & .. &.. & .. & .. & .. & .. & .. & .. & .. & $x$ & $x$ & $\mathrm{x}$ \\
\hline $\begin{array}{l}\text { ? Hippocrepina } \\
\text { Ammodiscidae }\end{array}$ & .. & .. & .. & . & " & $*$ & .. & .. & $x$ & .. & $x$ & $x$ & $\mathrm{x}$ \\
\hline Ammodiscus & .. & $x$ & $?$ & $?$ & $x$ & .. & .. & $x$ & $x$ & $x$ & $x$ & $x$ & $x$ \\
\hline Glomospirella & $?$ & $?$ & $?$ & $?$ & .. & $?$ & .. & $\mathrm{x}$ & $x$ & $x$ & $x$ & $x$ & .. \\
\hline Repmanina & $x$ & .. & ... & .. & .. & .. & .. & $\ldots$ & .. & .. & .. & .. & .. \\
\hline Tolypammina & .. & .. & ... & .. &.. & .. & .. & ... & .. & $x$ & .. & .. & .. \\
\hline
\end{tabular}

Note: Letters refer to the localities shown in Figure 1

2). Selected species whose identifications are uncertain or whose stratigraphic ranges seem important are illustrated in Plate 2. Although more species are included here than for any other order, they are generally rare and scattered in distribution. An exception is Astacolus cf. scitula, which is characterized by thick, but flush, sutures and occurs over a limited stratigraphic range.

\section{Order Buliminida}

Species belonging to the Bolivinidae, Turrilinidae, Fursenkoinidae, and Pleurostomellidae families are recorded (Table 3 ). Representatives of the Buliminida found here are among the most primitive of the order, and taxonomic problems occur in several groups. All species are illustrated in Plates 3 and 4.

The Bolivinidae family is represented by Tappanina sp., which seems more primitive than Tappanina laciniosa Eicher and Worstell recorded by Scheibnerová (1978a, Pl. 2, Fig. 11) from the Naturaliste Plateau. The species has a less lobate outline than Scheibnerová's specimen and lacks the carinate truncated chamber margins of the Coniacian-type species Tappanina selmensis (Cushman). Eicher and Worstell (1970) showed large variation in ornament for $T$. laciniosa from the North American CenomanianTuronian that ranged from well-developed peripheral flanges to smooth chamber surfaces. As recognized here, the genus is assumed to include forms having biserial chambers with simple sutures, but variable ornament and chamber shape, and a higharched aperture bordered on one side by a lip that extends internally as a simple tooth plate. This definition includes the Albian species attributed to Bolivina by Haig (1982). When the mid-Cretaceous bolivinids are better understood, we may find it meaningful to separate the Albian species from Tappanina on the basis of their less-truncate chamber shape.

The Turrilinidae family is represented by Cuneus sp., rare and confined to only the topmost samples, long-ranging Praebulimina nannina, and (?)Eurycheilostoma cf. hergottensis. Haig (1982, p. 47) tentatively transferred Praebulimina hergottensis to Eurycheilostoma on the basis of its trochospiral coiling in the juvenile stage and its very low and broad basal aperture. He also described a new species, Eurycheilostoma moorei, for low trochospiral morphotypes apparently evolved from $E$. hergottensis. In hindsight, the designation of these forms to Eurycheilostoma seems improbable, although one cannot completely dismiss this designation until the North American species of the genus are more completely described. Eurycheilostoma also was used by Sigal (1979, Pl. 3, Fig. 19) for a species similar to P. hergottensis. Coryphostoma sp. is a persistent and generally common faunal component in all studied assemblages, except for Sample 123$766 \mathrm{~A}-18 \mathrm{R}-2,83-85 \mathrm{~cm}$, where its absence is probably the result of dissolution. The species was recorded by Scheibnerová (1974b) from the Perth and Gascoyne Abyssal plains, and by Haig (1982) from the Laura, Carpentaria, and northeastern Eromanga basins in Queensland. Haig and Copp (unpubl. data) have also recorded the species from the Albian of the eastern Carnarvon Basin in western Australia. Among the Pleurostomellidae, the lowest occurrence of Pleurostomella aff. reu'ssi in Sample 123-766A-21R$\mathrm{CC}$ represents a significant stratigraphic level, as primitive $P$ leurostomella is not known elsewhere below the uppermost Aptian (Bettenstaedt and Spiegler, 1982). Ellipsoidella $\mathrm{cf}$. cuneata first appears in the stratigraphic sequence a little higher than Pleurostomella, and rare Ellipsoglandulina sp. occurs within Samples 123-766A-16R-5, 60-64 cm, and -16R-CC. The material offers no new evidence about the evolutionary development of the pleurostomellid genera.

\section{Order Rotalïda}

A remarkable diversification among the Rotaliida occurred during the mid-Cretaceous and resulted in a significant modernization of the fauna. Species recovered at Site 766 belong to the Patellinidae, Bagginidae, Quadrimorphinidae, Globorotalitidae, Osangulariidae, and Gavelinellidae families (Table 3). All species are illustrated in Plates 3 and 4.

Poorly preserved patellinids occur only in Samples 123-766A$24 \mathrm{R}-\mathrm{CC}$ and $-25 \mathrm{R}-1,33-35 \mathrm{~cm}$, and are referred tentatively to Hergottella. They lack the spinose umbonate deposits known in typical Hergottella from the Great Artesian Basin (Ludbrook, 1966 , p. 135,136 , Pl. 13, Figs. 19 through 22,27 , and 28 . Textfigs. 28 through 32 ; Haig, 1982 , p. 40,41 , Pl. 8 , Figs. 1 through 4).

The species classed as Serovaina and included in the Bagginidae are similar to mid-Cretaceous types referred elsewhere to Valvulineria or Gyroidinoides (e.g., Ludbrook, 1966; Dailey, 1973; Scheibnerová, 1974b, 1978a, 1978b; Sliter, 1977, 1986; Gradstein, 1978; Basov et al., 1979; Dupeuble, 1979; Haig, 1981, 1982; Basov and Krasheninnikov, 1983; Moullade, 1984; Magniez and Sigal, 1985). However, the species lack an open umbilicus and well-developed umbilical flaps, features typical of the 
Table 6. Distribution of foraminiferal genera of Lituolida in confirmed Aptian-Albian deposits of the western margin submarine plateaus and abyssal plains and the Australian epeiric basins.

\begin{tabular}{|c|c|c|c|c|c|c|c|c|c|c|c|c|c|}
\hline & \multicolumn{6}{|c|}{ WESTERN MARGIN } & \multicolumn{7}{|c|}{ EPEIRIC BASINS } \\
\hline & A & B & C & D & $\mathbf{E}$ & $\mathbf{F}$ & G & $\mathbf{H}$ & 1 & $J$ & $\mathbf{K}$ & L & M \\
\hline Rzehakinidae & & & & & & & & & & & & & \\
\hline $\begin{array}{l}\text { Miliammina } \\
\text { Hormosinidae }\end{array}$ & .. & $?$ & .. & .. & ... & .. &. & .. & $x$ & $\mathrm{x}$ & $x$ & $x$ & $x$ \\
\hline $\begin{array}{l}\text { Reophax } \\
\text { Haplophragmoldidae }\end{array}$ & .. & .. & .. & .. & .. & $?$ &.. & $x$ & $x$ & $x$ & $\mathrm{x}$ & $x$ & $x$ \\
\hline $\begin{array}{l}\text { Haplophragmoides } \\
\text { Lituolidae }\end{array}$ & .. & $x$ & $?$ & .. & $x$ & $?$ & .. & $x$ & $x$ & $\mathrm{x}$ & $x$ & $x$ & $\mathrm{x}$ \\
\hline Ammobaculites & .. & $?$ & .. & .. & $?$ & .. &.. & $x$ & $x$ & $x$ & $x$ & $x$ & $x$ \\
\hline Ammomarginulina & .. & .. & .. & .. & .. & .. & .. & .. & 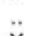 & .. & $x$ & . & .. \\
\hline Flabellammina & .. & .. & .. & .. & .. &. & .. & .. & $x$ &.. & $x$ & $x$ & $x$ \\
\hline Triplasia & .. & .. & .. & .. & .. & .. & .. & .. &.. & .. & .. & $x$ & .. \\
\hline $\begin{array}{l}\text { Sculptobaculites } \\
\text { Ammosphaeroidinidae }\end{array}$ & .. & .. & .. & .. & .. & $\cdot$. & .. & $\cdot \cdot$ & $x$ &.. & $x$ & $x$ & $\mathrm{x}$ \\
\hline Ammosphaeroidina & $x$ & $\ddot{~}$ & .. & .. & .. & .. & .. & .. &.. &.. & .. & .. & \\
\hline $\begin{array}{l}\text { Recurvoides } \\
\text { Spiroplectamminidae }\end{array}$ & .. & $?$ & .. & .. & .. & .. & .. & $\ddot{x}$ & $\ddot{x}$ & $\ddot{x}$ & $\ddot{x}$ & $\ddot{x}$ & $\ddot{x}$ \\
\hline $\begin{array}{l}\text { Spiroplectamminidae } \\
\text { ?Ammobaculoides }\end{array}$ &.. &.. & .. & .. & .. & . & & & & & & & \\
\hline Heterantyx & $\ddot{x}$ & .. & $\ddot{. .}$ & ? & .. & $?$ & $\begin{array}{l}. . \\
. .\end{array}$ & $\ddot{*}$ & $\ddot{*}$ & $\ddot{*}$ & $\mathrm{x}$ & $\mathrm{x}$ & 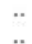 \\
\hline Spiroplectammina & .. & $?$ & $?$ & .. &.. & .. & ... & $\begin{array}{l}. . \\
. .\end{array}$ & $\ddot{x}$ & $\ddot{x}$ & $\ddot{x}$ & $\ddot{x}$ & $\ddot{x}$ \\
\hline $\begin{array}{l}\text { Spiroplectinella } \\
\text { Textulariopsidae }\end{array}$ & $x$ &.. & .. & .. & $?$ & $\ddot{*}$ & $\ddot{. .}$ & $\ddot{x}$ & .. & .. & .. & .. & .. \\
\hline Aaptotoichus & .. & .. & .. & .. &.. & ... & .. &.. & .. &.. & $x$ & $x$ & $x$ \\
\hline Bimonilina & .. & 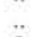 & .. &. & $?$ & .. &.. & $\ddot{. .}$ & $\ddot{x}$ & $\ddot{x}$ & $\hat{x}$ & $\hat{x}$ & $x$ \\
\hline $\begin{array}{l}\text { Textulariopsis } \\
\text { Trochamminidae }\end{array}$ & $\therefore$ & $?$ & .. & .. & .. & .*. & .. & $\ddot{x}$ & $x$ & $x$ & $x$ & $x$ & $\mathrm{x}$ \\
\hline $\begin{array}{l}\text { Trochammina } \\
\text { Prolixoplectidae }\end{array}$ & .. & $x$ & $?$ & .. &.. & $\ldots$ &.. & $x$ & $x$ & $x$ & $x$ & $x$ & $x$ \\
\hline $\begin{array}{l}\text { ?Eomarssonella } \\
\text { Verneuilinidae }\end{array}$ & $\cdots$ & .. & $\cdot$. & $\cdot$ & " & $"$ & .. & .. & $x$ & $x$ & $x$ & $x$ & $x$ \\
\hline Gaudryina & $\mathrm{x}$ & .. & $?$ & $?$ & $?$ & $?$ & .. & $\ddot{\ddot{z}}$ & .. &.. & .. & .. & .. \\
\hline Gaudryinella & .. & .. & .. & .. & .. & .. & .. & $x$ & $\ddot{\ddot{z}}$ & $\ddot{\ddot{z}}$ & $\ddot{\ddot{z}}$ & $\ddot{\ddot{x}}$ & $\ddot{z}$ \\
\hline Gaudryinopsis & $\ddot{\ddot{u}}$ & $\ddot{*}$ & $\ddot{~}$ & $\ddot{\ddot{z}}$ & .. & .. & .. & $x$ & $x$ & $\mathrm{x}$ & $x$ & $x$ & $\mathrm{x}$ \\
\hline Pseudogaudryinella & $\mathrm{x}$ & $\mathrm{x}$ & $?$ & $x$ & .. & .. & .. & .. & .. & $\ddot{\ddot{z}}$ &. & .. & .. \\
\hline ?Reophacella & .. & .. & .. & .. & .. & .. & .. & .. & $\ddot{\ddot{z}}$ & $x$ & .. & .. & .. \\
\hline Spiroplectinata & .. & .* & .. & .. & .. & .. & .. & $\ddot{\ddot{u}}$ & $x$ & $x$ &.. & .. & .. \\
\hline Uvigerinammina & .. & $\ddot{0}$ & $\ddot{n}$ & $\ddot{n}$ & $\ddot{0}$ & .. & .. & $x$ & $\ddot{z}$ & $\ddot{u}$ & $\ddot{z}$ & $\ddot{v}$ & $\ddot{v}$ \\
\hline Verneuilina & ... & $?$ & $?$ & $?$ & $?$ & .. & .. & .. & $x$ & $x$ & $x$ & $x$ & $\mathrm{X}$ \\
\hline $\begin{array}{l}\text { Verneuilinoides } \\
\text { Tritaxildae }\end{array}$ & ... & $?$ & .. & $?$ & .. & ... & ... & $x$ & $x$ &.. & $x$ & $x$ & $\mathrm{X}$ \\
\hline $\begin{array}{l}\text { Tritaxia } \\
\text { Globotextulariidae }\end{array}$ & $\mathrm{x}$ & .. & $?$ & $x$ & .. & $?$ & .. & ... & .. & .. & .. &. & .. \\
\hline $\begin{array}{l}\text { Remesella } \\
\text { Dictyopsellidae }\end{array}$ & $x$ & $x$ & $?$ & $x$ & .. & $?$ &.. & .. &.. &.. &.. & .. & .. \\
\hline $\begin{array}{l}\text { Andamookia } \\
\text { Eggerellidae }\end{array}$ & & & & & & & & .. &.. &.. &.. & $x$ & .. \\
\hline Dorothia & $x$ &.. & $?$ & $?$ & & $?$ & .. & .. & $\ddot{\ddot{n}}$ & $\ddot{x}$ & .. & .. & .. \\
\hline Marssonella & $x$ &.. &.. & .. & $?$ & $?$ & .. & .. & $x$ & $x$ & .. & .. & .. \\
\hline
\end{tabular}

Note: Letters refer to localities shown in Figure 1

optically radial Valvulineria and the optically granular Gyroidinoides. The studied specimens have transparent external walls and septa and may belong within the Bagginidae family. The globose Serovaina infracretacea is the most abundant and persistent species present in the sampled interval.

The lowest record of Osangularia schloenbachi occurs in Sample 123-766A-21R-CC, where it is abundant and possibly transitional from the compressed Gavelinella cf. andersoni. Osangularia schloenbachi remains a dominant species in the higher samples. Berthelina intermedia also first appears in Sample 123$766 \mathrm{~A}-21 \mathrm{R}-\mathrm{CC}$ and is persistent in all higher samples. The species is represented here by morphotypes that generally have a flatter oral side than those described by Haig (1982, p. 66 and 67) from northern Queensland (recorded as Anomalinoides intermedia). According to the diagnoses of Loeblich and Tappan (1988), the distinction between Anomalinoides and Berthelina and their separation in different families may lie only in the presence of more developed apertural flaps in Berthelina and a more extensive aperture reaching well across the periphery in Anomalinoides.
Four species of Gavelinella are recorded; each is characterized by an evolute or partially evolute spiral side and an involute umbilical side, with apertural flaps partly obscuring the umbilicus. Despite these similarities, the species appear unrelated in terms of chamber shape and the development of sutures, and probably were derived along different phylogenetic lines. None of the species shows a close relationship to Lingulogavelinella albiensis Malapris or to any other members of the Lingulogavelinella lineage described by Malapris (1965) and Malapris-Bizouard (1967).

The long-ranging Gavelinella $\mathrm{cf}$. andersoni was recorded as Planulina sp. by Haig (1981). This species is characterized by a very compressed test and thick, curved sutures. In the lower samples, a relationship may exist between this species and primitive Osangularia schloenbachi, with the latter species acquiring a more convex spiral side, a closed umbilicus, and a more confined aperture obliquely positioned at the base of the final chamber.

Gavelinella indica is confined to Samples 123-766A-20R-2, $48-52 \mathrm{~cm}$, and $-20 \mathrm{R}-3,68-72 \mathrm{~cm}$. The species was previously 
Table 7. Distribution of foraminiferal genera of Miliolida and Nodosariida in confirmed Aptian-Albian deposits of the western margin submarine plateaus and abyssal plains and the Australian epeiric basins.

\begin{tabular}{|c|c|c|c|c|c|c|c|c|c|c|c|c|c|}
\hline & \multicolumn{6}{|c|}{ WESTERN MARGIN } & \multicolumn{7}{|c|}{ EPEIRIC BASINS } \\
\hline & A & B & C & D & $\mathbf{E}$ & $\mathbf{F}$ & G & H & I & $J$ & K & $\mathbf{L}$ & M \\
\hline \multicolumn{14}{|l|}{ MILIOLIDA } \\
\hline \multicolumn{14}{|l|}{ Hauerinidae } \\
\hline Pseudosigmoilina & .. & $\ddot{~}$ &.. & .. & .. & .. & .. & $x$ & $x$ & .. & $x$ & $x$ & 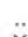 \\
\hline ?Scythiloculina & .. & $?$ & .. & ... & .. & .. & .. & .. & .. & .. & .. & $x$ & $\mathrm{x}$ \\
\hline \multicolumn{14}{|l|}{$\begin{array}{l}\text { NODOSARIIDA } \\
\text { Ichthyolariidae }\end{array}$} \\
\hline Bojarkaella & & & .. & .. & ., & & .. & $\cdots$ & .. & .. & .. & $x$ & $x$ \\
\hline Lingulonodosaria & $\ddot{x}$ & ? & .. & .. & .. & ? & .. & $\ddot{x}$ & $\ddot{x}$ & $x$ & $x$ & $x$ & $\mathrm{x}$ \\
\hline Nodosariliae & & & & & & & & & & & & & \\
\hline Dentalina & .. & .. & $?$ & $\ddot{*}$ & .. & ? & .. & .. & $x$ & $x$ &. & 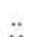 & .. \\
\hline Frondicularia & $?$ & $?$ & $?$ & $\mathrm{x}$ & .. & .. & .. & $x$ & $x$ & $x$ & .. & $x$ & .. \\
\hline ?Grigelis & $x$ & .. &.. &.. & .. & .. & .. &.. & .. & .. & .. & .. & .. \\
\hline Laevidentalina & $x$ & $x$ & $?$ & $\mathrm{x}$ & .. & $?$ & .. & $x$ & $x$ & $x$ & $x$ & $x$ & $\mathrm{x}$ \\
\hline Lingulina & $\mathrm{x}$ & .. & .. & .. & .. & ? & .. &.. & $x$ & $\mathrm{x}$ & $x$ & $x$ & .. \\
\hline ?Nodosaria & .. & .. & .. & .. & .. & .. & .. & ... & $x$ & $\mathrm{x}$ & .. & .. & .. \\
\hline Pseudonodosaria & $x$ & .. & .. & .. & .. & .. & $\mathrm{x}$ & $x$ & $x$ & $x$ & $\mathrm{x}$ & $\mathrm{x}$ & $\mathrm{x}$ \\
\hline Pyramidulina & $x$ & $\mathrm{x}$ & $?$ & $\mathrm{x}$ & ? & ? & .. & $x$ & $x$ & $\mathrm{x}$ & $\mathrm{x}$ & $\mathrm{x}$ & $x$ \\
\hline Svenia & $\mathrm{x}$ & .. & $?$ & $x$ & .. & .. & .. & .. & .. & $\mathrm{x}$ & $\mathrm{x}$ & $x$ & $\mathrm{x}$ \\
\hline Tristix & .. & .. & .. & ... & .. & .. & .. & .. & $x$ & $x$ & $\mathrm{x}$ & $\mathrm{x}$ & .. \\
\hline \multicolumn{14}{|l|}{ Vaginulinidae } \\
\hline Astacolus & $\mathrm{x}$ & $x$ & .. & $?$ & .. & ? & $x$ & $x$ & $x$ & $x$ & $x$ & $x$ & $x$ \\
\hline Citharina & $\mathrm{x}$ & $?$ &.. & .. & ? & .. & $x$ & $x$ & $\hat{x}$ & $\mathrm{x}$ & $x$ & $x$ & $\hat{x}$ \\
\hline ?Ellipsocristellaria & .. & .. & .. & ... & .. & ... & .. & .. & .. & $x$ & .. & .. & .. \\
\hline Hemirobulina & $\ddot{x}$ & $\ddot{x}$ & .. & $?$ & .. & ? & $x$ & $x$ & $\ddot{x}$ & $x$ & .. & $\mathrm{x}$ & $x$ \\
\hline Lenticulina & $x$ & $\mathrm{x}$ & ? & ? & $?$ & ? & $\mathrm{x}$ & $\mathrm{x}$ & $x$ & $\mathrm{x}$ & $x$ & $\mathrm{x}$ & $\mathrm{x}$ \\
\hline Marginulina & .. & $\hat{x}$ &.. & ? & $\therefore$ & ? & $x$ & $\hat{x}$ & $x$ & $\mathrm{x}$ & $\mathrm{x}$ & $\mathrm{x}$ & $\mathrm{x}$ \\
\hline Neoflabellina & $\ddot{. .}$ & .. & $\ddot{. .}$ & ? & ... & $\therefore$ & .. & ... & .. & .. & .. & .. & .. \\
\hline Planularia & $\ddot{x}$ & .. & .. & ? & .. & $\ddot{. .}$ & ... & $\ddot{x}$ & $\ddot{x}$ & .. & .. & .. & .. \\
\hline Psilocitharella & $x$ & $\ddot{*}$ & ... & $x$ &.. & $?$ & .. & .. & $x$ & $x$ & .. & $x$ & .. \\
\hline Saracenaria & $\mathrm{x}$ & $\ddot{x}$ & .. & ... & .. & ? & $\ddot{x}$ & $\ddot{x}$ & $\mathrm{x}$ & $x$ & $x$ & $x$ & $\mathrm{x}$ \\
\hline Vaginulina & $x$ &.. &.. & .. &. & ? & .. & .. & .. & .. & .. & .. & .. \\
\hline Vaginulinopsis & $\mathrm{x}$ & $x$ & $?$ &.. & $x$ & .. & & $x$ & $\mathrm{x}$ & $x$ & .. & $x$ & $x$ \\
\hline \multicolumn{14}{|l|}{ Lagenidae } \\
\hline Reussoolina & .. & .. & .. & .. & .. & ? & .. & $x$ & $x$ & $x$ & $x$ & $\mathrm{x}$ & $\mathrm{x}$ \\
\hline \multicolumn{14}{|l|}{ Polymorphinidae } \\
\hline Bullopora & .. &.. & .. & .. & .. & .. &.. &.. & $x$ & $x$ & .. & $\mathrm{x}$ & .. \\
\hline Eoguttulina & .. & $\mathrm{x}$ & .. & $?$ & .. & .. & .. & .. & .. & $x$ & .. & .. & .. \\
\hline Globulina & $x$ & $?$ & .. & .. & .. & $?$ & $x$ & $\mathrm{x}$ & $x$ & $x$ & $\mathrm{X}$ & $x$ & $\mathrm{x}$ \\
\hline Pyrulinoides & .. & .. & .. & .. & .. & ? & .. & .. & .. & $x$ & .. & .. & .. \\
\hline Ramulina & $\mathrm{x}$ & .. & ... & $x$ & .. & .. & .. & $x$ & $x$ & $x$ & .. & $x$ & .. \\
\hline \multicolumn{14}{|l|}{ Glandulinidae } \\
\hline Tricarinella & $x$ & .. & ... & $x$ & .. & .. & .. & $x$ & $x$ & $x$ & .. & $\mathrm{x}$ & .. \\
\hline
\end{tabular}

Note: Letters refer to localities shown in Figure 1.

referred to Orithostella by Scheibnerová (1974b, 1978b) and Narayanan and Scheibnerová (1975), and to Lingulogavelinella by Haig (1982). In contrast to typical Ortihostella, the spiral (aboral) side is completely evolute and usually flat to slightly concave. The initial spiral whorls may be obscured by granulate umbonate deposits (Pl. 4, Fig. 3).

The distinctive Gavelinella sp. A has a compressed test; thick, backwardly sweeping sutures; and well-developed apertural flaps that form a stellate pattern over the umbilicus. It occurs commonly over a narrow stratigraphic range from Sample 123-766A-19R$\mathrm{CC}$ to $-24 \mathrm{R}-\mathrm{CC}$.

Gavelinella sp. B is found in the interval from Sample 123$766 \mathrm{~A}-19 \mathrm{R}-4,23-27 \mathrm{~cm}$, to $-25 \mathrm{R}-\mathrm{CC}$, and becomes rare and more scattered toward the top of its range. This species is similar to the form described by Haig (1982) as Orithostella sp. A, based on a convex spiral (aboral) side and a flat, umbilical (oral) side. In typical Orithostella, the aboral side is completely involute, whereas in Gavelinella sp. B, it is partially evolute, with the early whorls clearly visible.

Scheibnerova protindica was established by Quilty (1984) for specimens from Exmouth Plateau. In Hole 766A, the species occurs as a common element of the fauna from Samples 123-
766A-16R-3, 46-50 cm, to -18R-CC. No direct ancestor for the species is apparent in studied assemblages. Stensioina sp. of Basov and Krasheninnikov (1983) may be synonymous with $S$. protindica.

\section{Order Globigerinida}

Planktonic foraminifers occur in all samples and include members of the Globigerinelloididae, Planomalinidae, Schackoinidae, Hedbergellidae, and Rotaliporidae families (Table 4). All species are illustrated in Plates 4 through 6.

The Globigerinelloides species are best represented in the lowest samples, although Globigerinelloides $\mathrm{cf}$. bentonensis is scattered higher in the hole. Three species are separated from Sample 123-766A-25R-CC: robust $G$. cf. bentonensis similar to morphotypes described by Caron (1978), with fewer chambers per whorl than in the holotype; small compressed Globigerinelloides aptiense, with six chambers in the final whorl; and related Globigerinelloides cf. aptiense, with five chambers in the last whorl. The Planomalina lineage is represented by Planomalina praebuxtorfi in Samples 123-766A-17R-2, 125-128 cm, and -17R-3, $36-39 \mathrm{~cm}$, and by Planomalina buxtorfi in the higher Samples 123-766A-16R-3, 46-50 cm, through -16R-CC. Planomalina 
Table 8. Distribution of foraminiferal genera of Robertinida, Buliminida, and Rotaliida in confirmed Aptian-Albian deposits of the western margin submarine plateaus and abyssal plains and the Australian epeiric basins.

\begin{tabular}{|c|c|c|c|c|c|c|c|c|c|c|c|c|c|}
\hline & \multicolumn{6}{|c|}{ WESTERN MARGIN } & \multicolumn{7}{|c|}{ EPEIRIC BASINS } \\
\hline & A & B & C & D & $\mathbf{E}$ & $\mathbf{F}$ & G & $\mathbf{H}$ & 1 & $J$ & $\mathbf{K}$ & $\mathbf{L}$ & M \\
\hline \multicolumn{14}{|l|}{$\begin{array}{l}\text { ROBERTINIDA } \\
\text { Ceratobuliminidae }\end{array}$} \\
\hline Ceratolamarckina & .. & .. & .. & .. & .. & .. & .. & .. & .. & .. & .. & $x$ & .. \\
\hline $\begin{array}{l}\text { Reinholdella } \\
\text { Epistominidae }\end{array}$ & .. & .. & .. & $\ldots$ & .. & .. & .. & .. & .. & .. & $x$ & $x$ & $x$ \\
\hline Epistomina & .. & .. & .. & .. & .. & .. & .. & $x$ & $x$ & $x$ & $x$ & $x$ & $x$ \\
\hline \multicolumn{14}{|l|}{ BULIMINIDA } \\
\hline Bolivinidae & & & & & & & & & & & & & \\
\hline $\begin{array}{l}\text { Tappanina } \\
\text { Eouvigerinidae }\end{array}$ & $\mathrm{x}$ & .. & .. & .. & .. & .. & .. & .. & .. & .. & $\mathrm{x}$ & $\mathrm{x}$ & .. \\
\hline $\begin{array}{l}\text { Eouvigerina } \\
\text { Turrilinidae }\end{array}$ & .. & .. & .. & .. &.. & .. & .. & .. & $x$ & .. & .. & .. & *. \\
\hline Cuneus & $x$ & .. & $\ddot{~}$ & $*$ & .. & .. & .* & .. & $\mathrm{x}$ & .. & $x$ & $\ddot{*}$ & $\ddot{n}$ \\
\hline Neobulimina & .. &.. & $?$ & & .. & $\because$. & $x$ & $x$ & $\mathrm{x}$ & $x$ & $x$ & $x$ & $x$ \\
\hline Praebulimina & $\ddot{x}$ & $\ddot{. .}$ & $\dot{x}$ & $?$ & $\ddot{. .}$ & ? & ... & $\hat{x}$ & ... & ... & ... & ... & .. \\
\hline Turrilina & .. & 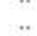 & ... &. & .. & .. & $\ddot{. .}$ & .. & $\ddot{x}$ & $\ddot{x}$ & $\mathrm{x}$ & $x$ & .. \\
\hline $\begin{array}{l}\text { ?Eurycheilostoma } \\
\text { Siphogenerinoididae }\end{array}$ & $\ddot{x}$ & $\ddot{*}$ & $\ddot{*}$ & ? & .. & $\ddot{*}$ & $\ddot{*}$ & $\ddot{. .}$ & $\hat{x}$ & $\hat{x}$ & $\mathrm{x}$ & $\mathrm{x}$ & $\mathrm{x}$ \\
\hline $\begin{array}{l}\text { Orthokarstenia } \\
\text { Buliminellidaee }\end{array}$ & .. & .. & .. & ... & .. & .. & .. & $x$ & $\mathrm{x}$ & $x$ & .. & $x$ & .. \\
\hline $\begin{array}{l}\text { ?Buliminella } \\
\text { Fursenkoinidae }\end{array}$ & .. & .. & .. & .. & .. & .. & .. & $x$ & $x$ & .. & $\mathrm{x}$ & $x$ & .. \\
\hline Cassidella & .. & .. & .. & .. &.. & .. &.. & .. & $x$ & $x$ & .. & .. & .. \\
\hline $\begin{array}{l}\text { Coryphostoma } \\
\text { Pleurostomellidae }\end{array}$ & $x$ &. & $?$ & $x$ & .. &.. & $\ddot{x}$ & .. & $x$ & $\mathrm{x}$ & .. & $\mathrm{x}$ & .. \\
\hline Ellipsoglandulina & $x$ & .. & .. & & .. & .. & .. & 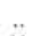 &.. & .. & .. & .. & .. \\
\hline Ellipsoidella & $x$ & .. & .. & $?$ & .. & .. & .. & $\mathrm{x}$ & $x$ & .. & .. & .. & .. \\
\hline Pleurostomella & $\hat{x}$ & .. & $\begin{array}{l}* . \\
. .\end{array}$ & $?$ & ? & $\ddot{?}$ & $\begin{array}{l}\ddot{.} \\
. .\end{array}$ & $\hat{x}$ & $\hat{x}$ & $\ddot{x}$ & $\begin{array}{l}. . \\
. .\end{array}$ & $\ddot{x}$ & .. \\
\hline
\end{tabular}

\begin{tabular}{|c|c|c|c|c|c|c|c|c|c|c|c|c|}
\hline \multicolumn{13}{|l|}{$\begin{array}{l}\text { ROTALIIDA } \\
\text { Spirillinidae }\end{array}$} \\
\hline $\begin{array}{l}\text { Turrispirillina } \\
\text { Patellinidae }\end{array}$ & .. & .. & .. & .. & .. & .. & .. & .. & .. & $x$ & .. & .. \\
\hline Hergottella & $?$ & .. & .. & $?$ & .. & .. & .. & .. & $x$ & .. & $x$ & $x$ \\
\hline Patellinoides & .. & .. & .. & .. & .. & .. & .. & $\ddot{x}$ & .. & $\ddot{x}$ & $\mathrm{x}$ & $\mathrm{x}$ \\
\hline Bagginidae & & & & & & & & & & & & \\
\hline Serovaina & $\mathrm{x}$ & .. & ? & $x$ & $\mathrm{x}$ & $x$ & .. & $x$ & $\mathrm{x}$ & $\mathrm{x}$ & $x$ & $x$ \\
\hline $\begin{array}{l}\text { Valvulinoides } \\
\text { Quadrimorphinidae }\end{array}$ & .. & .. & .. & .. & .. & $x$ & $\mathrm{x}$ & .. & $\mathrm{x}$ & $\mathrm{x}$ & $\mathrm{x}$ & $x$ \\
\hline $\begin{array}{l}\text { Quadrimorphina } \\
\text { Globorotalitidae }\end{array}$ & $x$ & .. & .. & $x$ & $x$ & .. & .. & $x$ & $x$ & $x$ & .. & .. \\
\hline $\begin{array}{l}\text { Globorotalites } \\
\text { Osangularidae }\end{array}$ & $x$ & .. & .. & $\mathrm{x}$ & $?$ & .. & .. & $x$ & .. & .. & .. & .. \\
\hline Charttonina & $x$ & .. & 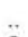 & .. & $x$ & 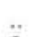 & .. & .. & .. & .. & .. & .. \\
\hline $\begin{array}{l}\text { Osangularia } \\
\text { Alabaminidae }\end{array}$ & $x$ & .. & ? & $\mathrm{x}$ & $?$ & $?$ & .. & $\mathrm{x}$ & .. & $x$ & .. & .. \\
\hline $\begin{array}{l}\text { ?Alabamina } \\
\text { Gavelinellidae }\end{array}$ & .. & ., & .. & .. & .. & $\mathrm{x}$ & .. & .. & .. & .. & .. & $\mathrm{x}$ \\
\hline Berthelina & $x$ & .. & ? & $\mathrm{x}$ & $x$ & $?$ & $x$ & $x$ & $x$ & $x$ & .. & $x$ \\
\hline Bilingulogavelinella & .. & .. & .. & .. & .. & .. & .. & .. & ... & ... & .. & $\mathrm{x}$ \\
\hline Gavelinella & $x$ & .. & ? & $x$ & .. & $x$ & $x$ & $\mathrm{x}$ & $x$ & $x$ & $x$ & $x$ \\
\hline Lingulogavelinella & .. & .. & $?$ & .. & $x$ & .. & .. & $x$ & $x$ & $x$ & $\mathrm{x}$ & $\mathrm{x}$ \\
\hline Scheibnerova & $x$ & .. & .. & .. & $\mathrm{x}$ & $\mathrm{x}$ & ... & ... & .. & ... & .. & .. \\
\hline
\end{tabular}

Note: Letters refer to localities shown in Figure 1.

buxtorfi (PI. 5, Figs. 8 and 9) is represented by the double-keeled variant discussed by Belford (1985, p. 185).

Species of Hedbergella are the most common planktonic forms in most samples. Hedbergella sp. cf. Blefuscuiana aptica, Hedbergella $\mathrm{cf}$. planispira, and Hedbergella planispira represent an evolving lineage through the lower and middle parts of the studied interval. All the species have granulate ornament and thus differ from species of the Praehedbergellidae Banner and Desai, 1988, which are smooth (Banner and Desai, 1988, p. 151). Hedbergella sp. cf. B. aptica has a high spire, four or five chambers in the final whorl, and distinct "perforation cones" (Pl. 5, Figs. 11 through 13). Within Sample 123-766A-25R-1, 33-35 cm, this species is transitional to low spired forms ( $H$. cf. planispira), with five or six chambers in the final whorl and with the final chambers often deflected slightly toward the umbilical side. In Samples 123766A-20R-2, 48-52 cm, and -20R-1, 47-57 cm, a gradation from $H$. cf. planispira to $H$. planispira occurs. The latter form possesses six or seven chambers in the final whorl and a flat spire. In previous literature, a variety of species was referred to $H$. planispira. The essential features of $H$. planispira are shown in Loeblich and Tappan's (1988, Pl. 495, Figs. 13 through 15) re-illustration of a topotype. This specimen has a flat spire, with six chambers in the final whorl; a thick test (with diameter:height ratio approximately $2: 1$ ); a diameter of approximately $0.23 \mathrm{~mm}$; 
Table 9. Distribution of foraminiferal genera of Globigerinida in confirmed Aptian-Albian deposits of the western margin submarine plateaus and abyssal plains and the Australian epeiric basins.

\begin{tabular}{|c|c|c|c|c|c|c|c|c|c|c|c|c|c|}
\hline & \multicolumn{6}{|c|}{ WESTERN MARGIN } & \multicolumn{7}{|c|}{ EPEIRIC BASINS } \\
\hline & A & B & C & D & $\mathbf{E}$ & $\mathbf{F}$ & G & H & 1 & $J$ & K & $\mathbf{L}$ & M \\
\hline Globigerinelloididae & & & & & & & & & & & & & \\
\hline $\begin{array}{l}\text { Globigerinelloides } \\
\text { Planomalinidae }\end{array}$ & $x$ & .. & $\mathrm{x}$ & .. & $?$ & .. & .. & .. & .. & .. & .. & .. & .. \\
\hline $\begin{array}{l}\text { Planomalina } \\
\text { Schackoinidae }\end{array}$ & $\mathrm{x}$ & .. & .. & .. & .. & $x$ & .. & $x$ & .. & .. & .. & .. & .. \\
\hline $\begin{array}{l}\text { Schackoina } \\
\text { Hedbergellidae }\end{array}$ & $\mathrm{x}$ & .. & .. & .. & $?$ & $x$ & .. & .. & .. & .. & $\mathrm{x}$ & .. & .. \\
\hline Hedbergella & $\mathrm{x}$ & .. & $\mathrm{x}$ & $x$ & $\mathrm{x}$ & $\mathrm{x}$ & $x$ & $x$ & $x$ & $x$ & $\mathrm{x}$ & $x$ & $\mathrm{x}$ \\
\hline Clavihedbergella & & .. & .. & $x$ & $x$ & $x$ & .. & $x$ & $x$ & .. & $\mathrm{x}$ & .. & .. \\
\hline $\begin{array}{l}\text { Praeglobotruncana } \\
\text { Favusellidae }\end{array}$ & $x$ & .. & .. & .. & $\mathrm{x}$ & .. & .. & $x$ & .. & .. & .. & .. & .. \\
\hline $\begin{array}{l}\text { Favusella } \\
\text { Rotaliporidae }\end{array}$ &.$\cdot$ & 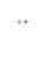 & .. & .. & .. & .. & .. & $\mathrm{x}$ & .. & .. & .. & .. & .. \\
\hline Rotalipora & $x$ & .. & .. & & .. & .. & .. & $\mathrm{x}$ & .. & .. & .. & .. & .. \\
\hline
\end{tabular}

Note: Letters refer to localities shown in Figure 1.

granulate ornament on both the spiral and umbilical sides; and a wide deep umbilicus. These features are present in the Site 766 specimens and in those recorded by Playford et al. (1975) from the "Great Artesian Basin." The specimens identified from DSDP sites as H. planispira by Herb (1974), Gradstein (1978), Caron (1978), and Miles and Orr (1980) are not conspecific with the topotype figured by Loeblich and Tappan. These belong to an unrelated species referred here to Hedbergella cf. punctata (following the descriptions of Michael, 1972, and Playford et al., 1975). Hedbergella cf. punctata is abundant in Samples 123$766 \mathrm{~A}-17 \mathrm{R}-\mathrm{CC}$ to $-16 \mathrm{R}-3,46-50 \mathrm{~cm}$; below this level, it is rare and scattered. The species is characterized by a very small $(<0.2$ $\mathrm{mm}$ diameter) flat trochospire having a smooth surface and large scattered pores, and seven chambers in the final whorl.

Although Hedbergella trocoidea is represented by typical specimens, its scattered distribution probably does not reflect the species complete stratigraphic range. Unornamented Hedbergella yezoana is the same species recorded by Playford et al. (1975) as Hedbergella sp. A, and by Herb (1974) as Ticinella sp. A. The species may be related to Ticinella primula Luterbacher, but lacks supplementary apertures. In Hole $766 \mathrm{~A}$, it occurs in Samples 123-766A-19R-CC through -16R-3, 46-50 cm.

Loeblich and Tappan (1988) placed Clavihedbergella in Family Rotaliporidae. Here, the species is considered to belong to the Hedbergellidae family because it lacks the supplementary sutural apertures typical of the Rotaliporidae. Two species are represented: small, smooth-shelled Clavihedbergella sp. seems a primitive species of the genus and has its lowest occurrence in Sample 123-766A-20R-CC; and the larger spinose Clavihedbergella simplicissima first appears higher in the succession in Sample 123 766A-17R-5, 29-32. Among the Sample 123-766A-16R-5, 60-64 $\mathrm{cm}$, specimens are atypical morphotypes having six chambers in the final whorl.

The most advanced specimens of the Family Hedbergellidae encountered here are referred to Praeglobotruncana delrioensis and occur only in the uppermost samples (123-766A-16R-CC through $-16 \mathrm{R}-3,46-50 \mathrm{~cm})$. Some of these specimens are very strongly ornamented with large pustules, which are variably concentrated around the periphery (Pl. 6, Figs. 5 and 6).

Rotalipora is represented by two species having concurrent ranges in Samples 123-766A-16R-5, 60-64 cm, and -16R-3, 46$50 \mathrm{~cm}$. Both have strong keels on the spiral side, and ridges variously developed along the chamber margins and umbilical shoulders on the umbilical side. Rotalipora appenninica has an asymmetric test (more convex on umbilical side) with six or seven chambers in the final whorl and may, on the basis of periumbilical ridge development, be an advanced morphotype of the species, tending toward $R$. greenhornensis (Morrow) and resembling $R o$ talipora globotruncanoides Sigal (see Wonders, 1980, p. 127, 128). Rotalipora ticinensis also has stronger ornament than is typical for the species; it has seven or eight chambers in the final whorl, and the spiral side often is more convex than the umbilical side.

\section{Implications for Chronostratigraphy}

The chronostratigraphic subdivision of Hole $766 \mathrm{~A}$ interval from Cores $123-766 \mathrm{~A}-16 \mathrm{R}$ through $-25 \mathrm{R}$, based on foraminiferal biostratigraphy, is shown in Figure 2. Planktonic foraminiferal zones of wide application are applied only to Core 123-766A-16R and the upper part of Core 123-766A-17R. Here, the Planomalina praebuxtorfi Zone of Wonders $(1980 ;=$ Planomalina praebuxtorfi Subzone of Leckie, 1984), defined by the total range of the nominate species, occupies Sections 123-766A-17R-2 and -17R-3; and the succeeding undifferentiated Pseudothalmanninella ticinensis-Planomalina buxtorfi and Thalmanninella appenninicaPlanomalina buxtorfi zones of Wonders (1980) range through Sections $123-766 \mathrm{~A}-16 \mathrm{R}-3$ to $123-766 \mathrm{~A}-16 \mathrm{R}-5$, based on the occurrence of $P$. buxtorfi. The entire interval (Sections 123-766A16R-3 to -17R-3) correlates with the Rotalipora appenninica Zone of Caron (1985) and with the Stoliczkaia disparammonoid Zone indicative of the upper Albian (Caron, 1985, Fig. 3). In Hole $766 \mathrm{~A}$, Rotalipora is confined to the upper part of this interval. The presence of advanced Rotalipora appenninica tending toward Rotalipora globotruncanoides suggests that the uppermost sample lies close to the Albian/Cenomanian boundary (Wonders, 1980).

The interval below Core $123-766 \mathrm{~A}-17 \mathrm{R}$ is difficult to correlate precisely with standard chronostratigraphic divisions, because most of the commonly used zonal index species do not occur. The lowest samples from Cores 123-766A-24R and -25R are probably upper Aptian, based on (1) the abundance of Hedbergella sp. cf. Blefuscuiana aptica among the planktonic foraminifers, the occurrence in Core 123-766A-25R of Globigerinelloides aptiensis, and the first occurrence of abundant Hedbergella cf. planispira at the top of Core 123-766A-25R; and (2) the absence of benthic species such as Berthelina intermedia, Osangularia schloenbachi, and Pleurostomella aff. reussi. Unfortunately, no samples were taken from Cores 123-766A-22R and -23R because of poor core recovery in an apparently siliceous interval (? correlative of 
the upper Aptian-lower Albian Windalia Radiolarite of the Carnarvon Basin; Hocking 1988; and Ellis and Haig, unpubl. data).

The foraminiferal assemblages recovered from Core 123766A-21R are markedly different from those of the lower cores. Berthelina, Osangularia, and Pleurostomella appear as common elements of the benthic fauna. The planktonic assemblages are dominated by Hedbergella cf. planispira and include scattered Hedbergella trocoidea. Because of the faunal contrast with the lower cores, Core 123-766A-21R is taken as basal Albian, although it cannot be correlated precisely. The development of Hedbergella assemblages in the interval ranging from Core 123 766A-21R upward to Core $123-766 \mathrm{~A}-17 \mathrm{R}$ is broadly similar to that recorded in the "Great Artesian Basin" by Playford et al. (1975) and Haig (1979a). There, the lower and middle Albian assemblages are dominated by Hedbergella planispira, whereas the upper Albian fauna includes Hedbergella cf. punctata, Hedbergella yezoana, Clavihedbergella sp., and Hedbergella delrioensis (not recognized in Hole 766A). Therefore, the middle Albian/upper Albian boundary seems to lie somewhere within Core 123-766A-18R and has been arbitrarily placed between Sections 123-766A-18R-3 and -18R-5 on the basis of a decreased abundance of $H$. planispira up the sequence and a corresponding increased abundance of $H$. cf. punctata.

\section{COMPARISON OF FAUNA}

The Site 766 record described above adds significantly to our knowledge of the fauna in oceanic sediment bordering the western continental margin of Australia. It is now appropriate to review the known Australian fauna to clarify further the differences in composition among the assemblages that inhabited the shallow epeiric seas and those that lived along the continental margin. Global biogeographic relationships are apparent only when the Australian fauna is viewed in overall aspect. Haig (1979a, 1979b, 1980, 1982) described the ecological distribution of benthic species in the "Great Artesian Basin" by recognizing faunal associations (viz. Ammobaculites Association of the restricted epeiric sea, and the Marssonella Association of the open continental margin). Each association is characterized by different species. The most striking difference among the associations occurs in the composition of the agglutinated fauna: calcareous agglutinated types are absent from the Ammobaculites Association (even where diverse nodosariid, robertinid, buliminid, and rotaliid assemblages occur), whereas calcareous agglutinated foraminifers are common in the Marssonella Association. Within the Ammobaculites Association, different biofacies (characterized by different suites of species) reflect an increasing water depth: marginal marine Ammobaculites australis biofacies; inner sublittoral Lingulogavelinella albiensis biofacies; and outer sublittoral Neobulimina albertensis biofacies.

The Site 766 benthic assemblages belong to the Marssonella Association, as do the benthic assemblages described by Scheibnerová (1974b, 1978a) and Quilty (1984) from other localities on the western margin submarine plateaus and abyssal plains. Elsewhere in the Australian region, the Marssonella Association is known from Albian deposits of the Laura and northern Carpentaria basins (Haig, 1979a) and from part of the Albian succession in the Papuan Basin (Haig, 1981; Belford, 1985). The Ammobaculties Association is known from Aptian-Albian deposits of the Surat Eromanga and southern Carpentaria basins and from Aptian deposits of the Laura and northern Carpentaria basins (Haig, 1979a). Ammobaculites Association faunas containing some species more typical of the Marssonella Association occur in lowermiddle Albian deposits of the northeastern Eromanga Basin (Haig, 1979a) and in middle Albian strata of the eastern Carnarvon Basin (Haig and Cop, unpubl. data).
Analysis of the Argo Abyssal Plain fauna of Aptian-Albian age recovered from Site 765 (Ludden, Gradstein, et al., 1990) is incomplete, and the faunal records are not included in the distribution tables. One hopes that assemblages from the Argo Abyssal Plain will clarify the nature of the abyssal fauna ("Recurvoides Association" of Haig, 1979) equivalent to that found by Krasheninnikov (1974b) in Upper Cretaceous strata at DSDP Site 261.

The generic classification outlined by Loeblich and Tappan is used here (Tables 5 through 9), in part to see whether the generic taxa provide a suitable basis for representing the differences in faunal diversity among regions on the continent. Tables 5 through 9 record the occurrences of genera based on species documented in published studies and our unpublished data. For ease of comparison, the regions have been grouped as (1) western margin submarine plateaus and abyssal plains and (2) epeiric basins. This comparison has been undertaken separately for each foraminiferal order.

\section{Order Astrorhizida}

Table 5 documents the genera known from the Australian region. The Ammobaculites Association in the epeiric basins contains a varied assortment of genera, with the species characterized by noncalcareous walls. Many of these species are remarkably similar to those known from coeval flysch deposits. This may reflect a similar adaptation to low-oxygen conditions in muddy environments independent of water depth.

In contrast to the rich diversity of the epeiric basin assemblages, the known western margin faunas contain a sporadic record of genera belonging mainly to the Ammodiscidae.

\section{Order Lituolida}

Table 6 charts the distribution of genera known from the Australian region. The most diverse assemblages occur within the Ammobaculites Association of the epeiric basins. Here, all species have noncalcareous walls. In contrast, most of the lituolids from the Marssonella Association have calcareous material incorporated in their tests. The generic classification emphasizes the difference among the faunal associations. Genera such as Ammosphaeroidina, Heterantyx, Spiroplectinella, calcareous Textulariopsis, Gaudryina, Pseudogaudryinella, Spiroplectinata, Tritaxia, Remesella, Dorothia, and Marssonella are confined to the Marssonella Association. The dominant genera of the Ammobaculites Association are Miliammina, Reophax, Haplophragmoides, Ammobaculites, Sculptobaculites, Recurvoides, Spiroplectammina, Aaptotoichus, Bimonilina, noncalcareous Textulariopsis, Trochammina, (?)Eomarssonella (recorded as Riyadhella by Haig, 1979a, 1980), Gaudryinopsis, Verneuilina, and Verneuilinoides. In terms of reflecting faunal diversity, this classification works, but may be improved by separating the calcareous and noncalcareous Textulariopsis into different genera.

\section{Order Miliolida}

Two genera are present in the epeiric basin faunas (Table 7). Pseudosigmoilina and (?)Scythiloculina occur in the Ammobaculites Association, whereas only Pseudosigmoilina is present in the Marssonella Association of northern Queensland. Mililiolids are recorded from only one site on the western margin, which suggests that the genera were confined mostly to shallower seas.

\section{Order Nodosariida}

Compared with the other foraminiferal orders, much more correspondence exists here between faunas of the western margin and the epeiric basins (Table 7), and between the Ammobaculites and Marssonella associations. Many of the species seem to have long stratigraphic ranges, and through time may have adapted progressively to a wide range of environmental conditions (this 
hypothesis should be tested, especially among Jurassic assemblages that contain the ancestral forms).

Genera mostly confined to the Marssonella Association include Dentalina (now circumscribed for costate species), Frondicularia, Planularia (with carinate periphery), Psilocitharella, and Tricarinella (as distinct from Tristix).

\section{Order Robertiniida}

Three genera are recorded from the Ammobaculites Association of the epeiric basins (Table 8). None are recorded from the Marssonella Association. This may be because of post-burial dissolution of the aragonite tests within permeable carbonate-rich sediments that contain many of the Marssonella Association assemblages, particularly along the western margin.

\section{Order Buliminida}

The Australian region contains a splendid record of primitive Buliminida (Table 8). The Ammobaculites Association is particularly rich in Neobulimina and (?)Eurycheilostoma and contains some of the earliest records of Turrilina, Tappanina, and a form resembling Buliminella. Genera more typical of the Marssonella Association include Eouvigerina, Orthokarstenia, Cassidella, Coryphostoma, Ellipsoglandulina, Ellipsoidella, and Pleurostomella.

\section{Order Rotaliida}

Table 8 is a record of genera known from the Australian region. Serovaina and Gavelinella, which include dominant species at Site 766 , occur in all regions and are spread throughout the Marssonella and Ammobaculites associations. Quadrimorphina, Osangularia, and Berthelina are widely represented only in the Marssonella Association. The occurrence of rare Globorotalites in western margin deposits and in the northern Papuan Basin suggests that this genus was confined to deep bathyal conditions in the Australian region. Scheibnerova and Charltonina are confined to the western margin, although these small forms may have been overlooked elsewhere, especially among poorly preserved material such as occurs in the Papuan Basin. Lingulogavelinella and Bilingulogavelinella are best represented within the Ammobaculites Association of the epeiric basins.

\section{Order Globigerinida}

The record of planktonic genera (Table 9) shows an apparent marked reduction in faunal diversity existing among regions near the continental margin and those farther within the epeiric basins. However, care is needed in this comparison because marked changes in planktonic diversity occurred during Aptian-Albian time in this region. The most diverse faunas are from uppermost Albian deposits along the western margin and in the Papuan Basin; these are compared in more detail later in the text. Uppermost Albian deposits in the Laura, Carpentaria, Eromanga, and Surat basins record a regressive marine phase and lack planktonic foraminifers.

\section{Implications for Global Biogeography}

During the Early Cretaceous, the Australian region lay at middle to high paleolatitudes in the Southern Hemisphere, higher than $35^{\circ} \mathrm{S}$, at least for the Berriasian through Aptian, according to the reconstructions of $\mathrm{Ogg}$ et al. (this volume). Because of the high-latitude position of the Australian continent and the nature of the fine siliciclastic sediment that accumulated within the epeiric seas, it may be that three main environmental factors influenced foraminiferal diversity in this region: cool water temperatures (emphasized by Scheibnerová, 1986); hyposalinity in the epeiric seas (emphasized by Haig, 1979a); and low oxygen levels in the stagnant, silled, bottom waters of the shallow epeiric seas.

Haig (1979b) outlined the broad global distribution patterns of the Ammobaculites and Marssonella associations during the midCretaceous (based on an analysis of species later published by Haig, 1980, 1982). The Ammobaculites Association is represented mainly in the epeiric basins of Australia, western Siberia, and western interior North America by markedly similar faunas; whereas the Marssonella Association is well known from most continents in marginal basins that had open access to the ocean. The Marssonella Association is more variable in terms of generic diversity among regions.

Australian genera rarely recorded elsewhere in Aptian-Albian strata include the dictyopsellid Andamookia (known only from the Ammobaculites Association of the southern Eromanga Basin), the patellinid Hergottella (from both the Ammobaculites and Marssonella associations), the eouvigerinid Eouvigerina (from the Marssonella Association), the fursenkoinids Cassidella and $\mathrm{Co}$ ryphostoma (from the Marssonella Association); the gavelinellids Bilingulogavelinella (from the Ammobaculites Association), and Scheibnerova (from the Marssonella Association).

Families that are missing from the Australian fauna, but are recorded elsewhere from Aptian-Albian deposits, include Mayncinidae, Nezzazatidae, Barkerinidae, Charentiidae, Coscinophragmatidae, Cyclolinidae, Cyclamminidae, Ataxophragmiidae, Cuneolinidae, Dicyclinidae, Pfenderinidae, Orbitolinidae, Chrysalidinidae, Involutinidae, Alveolinidae, Conorboididae, Heteroheliicidae, Caucasinidae, Conorbinidae, Cibicididae, Chilostomellidae, and Karreriidae. Many of these represent "larger" complex foraminifers from shelf carbonate deposits of the warmwater Tethyan belt (Dilley, 1971).

The absence of Ataxophragmiidae from the Australian fauna is interesting because of its common occurrence in Boreal assemblages (Price, 1975; Barnard and Banner, 1980 ). Planktonic Ticinella (Rotaliporidae) also has not been definitely identified from the Australian material, although closely related Hedbergella species are abundant. The record of Ticinella multiloculata by Quilty (1984, Fig. 3D) was not confirmed by his illustration of only the dorsal (spiral) side; and Ticinella was not recorded by Herb (1974) from the Naturaliste Plateau DSDP Site 258. However, at DSDP Site 257 in the Wharton Basin to the northwest, Herb (1974, p. 753, Pl. 2, Fig. 17) noted one specimen that had a supplementary aperture on the final chamber that is typical of Ticinella.

Thus, the Australian fauna contains few genera that are not recorded elsewhere, but lacks a multitude of Tethyan families found in the paleoequatorial belt. This decrease in diversity from the equatorial region south probably reflects a temperature gradient (as Scheibnerová, 1970, 1986; Dilley, 1971; and Haig, 1979b; deduced). A relative measure of this gradient, at least for the latest Albian, may be obtained by comparing planktonic assemblages found along the continental margin from the Papuan Basin in the north, through Site 766 (at $5^{\circ}$ farther south, according to reconstructions of Veevers et al., 1991), to DSDP Site 258 on the Naturaliste Plateau, approximately $10^{\circ}$ south of Site 766 . The late Albian fauna of the Papuan Basin is almost identical to that from Site 766 , but contains rare Favusella not present in western margin assemblages. Favusella apparently has an aragonitic wall (Banner and Desai, 1988) that makes it more vulnerable to dissolution than other planktonic foraminifers having calcitic tests, and its distribution must be viewed with caution when making biogeographic comparisons, such as those of Koutsoukos et al. (1989). A uniform planktonic fauna having abundant Planomalina, Rotalipora, Praeglobotruncana, Clavihedbergella, and Hedbergella (but not Ticinella) may have occupied the latitudinal range between the Papuan Basin and Site 766 during the latest Albian. To 
the south, a marked decrease in species diversity accompanies the $10^{\circ}$ paleolatitude difference between Sites 766 and 258. The Site 258 fauna, associated with nannoplankton of the upper Albian Eiffellithus turriseiffeli Zone (Thierstein, 1974), contains only rare Praeglobotruncana delrioensis, Clavihedbergella simplicissima, Hedbergella delrioensis, and "Hedbergella planispira"; Rotalipora and Planomalina are absent (Herb, 1974). This faunal change probably was the result of a marked decrease in surfacewater temperatures along the western continental margin between Site 766 and the Naturaliste Plateau.

\section{CONCLUSIONS}

The foraminifers recovered from Core 123-766A-25R through Section 123-766A-16R-3 suggest that this portion of Hole $766 \mathrm{~A}$ ranges from the upper Aptian to the uppermost Albian and is coeval with deposits that accumulated on the Australian continent during an extensive marine transgression. The benthic assemblages from Site 766 belong to the Marssonella Association and are similar to those recorded elsewhere along the continental margin, but differ from assemblages found within more restricted parts of the epeiric basins (viz., the Ammobaculites Association). In a global comparison, the Australian fauna contains few genera not recorded elsewhere, but lacks many families present in Tethyan (equatorial) faunas. Three main environmental factors apparently influenced foraminiferal diversity in the Australian region: (1) cool water temperatures, (2) hyposalinity in the epeiric seas, and (3) low oxygen levels in the stagnant, silled, bottom waters of the shallow epeiric seas. Comparison of late Albian planktonic foraminifers known from along the continental margin indicates that a significant gradient in surface-water temperature existed over a $10^{\circ}$ latitudinal range between Cuvier Abyssal Plain Site 766 and Naturaliste Plateau Site 258 on the late Albian continental margin.

\section{ACKNOWLEDGMENTS}

I am grateful to the Ocean Drilling Program for inviting me to participate as a shipboard scientist during Leg 123. I thank my other shipboard colleagues and the captain, crew, and technical staff of the JOIDES Resolution for providing such an interesting and pleasant cruise. Sincere thanks are due to Patrick Coleman, Felix Gradstein, and Mark Leckie for their helpful reviews of the manuscript. I am grateful to the staff of the Electron Microscopy Centre of the University of Western Australia for facilitating my use of a scanning electron microscope. The University of Western Australia provided financial support for travel and sample analysis, and the Australian Secretariat of the Ocean Drilling Program funded my attendance at the post-cruise meeting in College Station.

\section{REFERENCES}

Apthorpe, M. C., 1979. Depositional history of the Upper Cretaceous of the northwest shelf, based upon foraminifera. APEA J., 19:74-89.

Banner, F. T, and Desai, D., 1988. A review and revision of the JurassicEarly Cretaceous Globigerinina, with especial reference to the Aptian assemblages of Speeton (North Yorkshire, England). J. Micropalaeontol., 7:143-185.

Barnard, T. and Banner, F. T., 1980. The Ataxophragmiidae of England: Part 1, Albian-Cenomanian Arenobulimina and Crenaverneuilina. Rev. Española Micropaleontol., 12:383-430.

Bartenstein, H., 1974. Upper Jurassic-Lower Cretaceous primitive arenaceous foraminifera from DSDP Sites 259 and 261, eastern Indian Ocean. In Veevers, J. J., Heirtzler, J. R., et al., Init. Repts. DSDP, 27: Washington (U.S. Govt. Printing Office), 683-688.

Basov, I. A., and Krasheninnikov, V. A., 1983. Benthic foraminifers in Mesozoic and Cenozoic sediments of the southwestern Atlantic as an indicator of paleoenvironment, Deep Sea Drilling Project Leg 71. In Ludwig, W. J., Krasheninnikov, V. A., et al., Init. Repts. DSDP, 71: Washington (U.S. Govt. Printing Office), 739-788.

Basov, V. A., Lopatin, B. G., Gramberg, I. S., Danjushevskaya, A. I., Kaban'kov, V. Ya., Lazurkin, V. M., and Patrunov, D. K., 1979. Lower Cretaceous lithostratigraphy near Galicia Bank. In Sibuet, J.-C., Ryan, W.B.F., et al., Init. Repts. DSDP, 47(2): Washington (U.S. Govt. Printing Office), 683-717.

Belford, D. J., 1985. Late Albian planktonic foraminifera, Strickland River, Papua New Guinea. BMR J. Australian Geol. Geophys., 9:183189.

Berthelin, G., 1880. Mémoire sur les Foraminifères fossiles de l'Etage Albien de Moncley (Doubs). Mém. Soc. Géol. France, Ser. 3, 1(5): $1-84$.

Bettenstaedt, F., 1952. Stratigraphisch wichtige Foraminiferen-Arten aus dem Barrême vorwiegend Nordwest-Deutschlands. Senckenbergiana, 33:263-295.

Bettenstaedt, F., and Spiegler, D., 1982. Pleurostomella (Foram.) in der Unterkreide Nordwestdeutschlands. Geol. Jhrb. (Hanover), Reihe A, 65:445-479.

Carbonnier, A., 1952. Sur un gisement de foraminifères d'âge Cénomanien supérieur de la région de Taza (Maroc), Bull. Soc. Géol. France, Sér. 6. 2:111-122.

Caron, M., 1978. Cretaceous planktonic foraminifers from DSDP Leg 40, southeastern Atlantic Ocean. In Bolli, H. M., Ryan, W.B.F., et al., Init. Repts. DSDP, 40: Washington (U.S. Govt. Printing Office), 651-678.

1985. Cretaceous planktonic foraminifera. In Bolli, H. M., Saunders, J. B., and Perch-Nielsen, K. (Eds.), Plankton Stratigraphy: Cambridge (Cambridge Univ. Press), 17-86.

Chapman, F., 1894. The foraminifera of the Gault of Folkestone, Part VII. J. R. Microscopical Soc., 1894:645-654.

Church, C. C., 1968. Lower Cretaceous foraminifera of the Orchard Peak-Devils Den area, California. Proc. California Acad.Sci., Ser. 4 , 32:523-580.

Crespin, I., 1944. Some Lower Cretaceous foraminifera from bores in the Great Artesian Basin, northern New South Wales. Proc. J. R. Soc. New South Wales, 78:17-24.

1953. Lower Cretaceous foraminifera from the Great Artesian Basin, Australia. Contrib. Cushman Found. Foramin. Res., 4:26-36.

1963. Lower Cretaceous arenaceous foraminifera of Australia. Bull. BMR Geol. Geophys., Australia, 66.

Crittenden, S., 1983. Osangularia schloenbachi (Reuss, 1863): an index foraminiferid species from the Middle Albian to Late Aptian of the southern North Sea. Neues Jhrb. Geol. Paläontol. Abh., 167:40-64.

Cushman, J. A. and Jarvis, P. W., 1928. Cretaceous foraminifera from Trinidad. Contrib. Cushman Lab. Foramin. Res., 4:85-103.

Dailey, D. H., 1970. Some new Cretaceous foraminifera from the Budden Canyon Formation, northwestern Sacramento Valley, California. Contrib. Cushman Found. Foramin. Res., 21:100-111.

, 1973. Early Cretaceous foraminifera from the Budden Canyon Formation, northwestern Sacramento Valley, California. Univ. California Publ. Geol. Sci., 106.

Dam, A. ten, 1947. Sur quelques espèces nouvelles ou peu connues dans le Crétacé inférieur (Albien) des Pays-Bas. Geol. Mijnb., 26:25-29.

1950. Les foraminifères de l'Albien des Pays-Bas. Mém. Soc. Géol. France, 63.

Dilley, F. C., 1971. Cretaceous foraminiferal biogeography. Spec. Issue, Geol.J., 4:169-190.

Dupeuble, P. A., 1979. Mesozoic foraminifers and microfacies from Holes 400A, 401 and 402A of the DSDP Leg 48. In Montadert, L., Roberts, D. G., et al., Init. Repts. DSDP, 48: Washington (U.S. Govt. Printing Office), 451-473.

Dzhafarov, D. I., Agalarova, D. A., and Khalilov, D. M., 1951. [Handbook for the microfauna of the Cretaceous deposits of Azerbaidzhan]: Baku, USSR (Aznefteizdat). (fide Ellis and Messina)

Eichenberg, W., 1935. Die Erforschung der Mikroorganismen insbesondere der Foraminiferen der norddeutschen Erdölfelder; Teil 1 - Die Foraminiferen der Unterkreide; Folge 4 - Foraminiferen aus dem Apt von Wenden am Mittellandkanal. Nieder. Geol. Verein, Jahb., 27:140. 
Eicher, D. L. and Worstell, P., 1970. Cenomanian and Turonian Foraminifera from the Great Plains, United States. Micropaleontology. $16: 269-324$.

Ellis, B. F., and Messina, A. R., 1940 et seq. Catalogue of Foraminifera. Am. Museum Natural History, Spec. Publ.

Frakes, L. A., Burger, D., Apthorpe, M., Wiseman, J., Dettmann, M., et al. (Australian Cretaceous Palaeoenvironments Group), 1987. Australian Cretaceous shorelines, stage by stage. Palaeogeogr., Palaeoclimatol., Palaeoecol., 59:31-48.

Gandolfi, R., 1942. Ricerche micropaleontologiche e stratigrafiche sulla scaglia e sul flysch Cretacici dei Dintorni di Balerna (Canton Ticino). Rivi. Italiana Paleontol., Mem. 4 48:1-160.

Grabert, B., 1959. Phylogenetische Untersuchungen an Gaudryina und Spiroplectinata (Foram.) besonders aus dem nordwestdeutschen Apt und Alb. Senckenberg. Naturfor. Gesell., Abh., 498:1-71.

Gradstein, F. M., 1978. Biostratigraphy of Lower Cretaceous Blake Nose and Blake-Bahama Basin foraminifers DSDP Leg 44, western north Atlantic Ocean. In Benson, W. E., Sheridan, R. E., et al., Init. Repts. DSDP , 44: Washington (U.S. Govt. Printing Office), 663-701.

Haig, D. W., 1979a. Cretaceous foraminiferal biostratigraphy of Queensland. Alcheringa, 3:171-187.

1979b. Global distribution patterns for mid-Cretaceous foraminiferids. J. Foramin. Res., 9:29-40.

1980. Early Cretaceous textulariine foraminiferids from Queensland. Palaeontographica A, 170:87-138.

1981. Mid-Cretaceous foraminiferids from the Wahgi Valley, Central Highlands of Papua New Guinea. Micropaleontology, 27: $337-351$.

1982. Early Cretaceous milioline and rotaliine benthic foraminiferids from Queensland. Palaeontographica A, 177:1-88.

Haig, D. W., and Barnbaum, D., 1978. Early Cretaceous microfossils from the type Wallumbilla Formation, Surat Basin, Queensland. Alcheringa, 2:159-178.

Haynes, J. R., 1981. Foraminifera: London (MacMillan).

Herb, R., 1974. Cretaceous planktonic foraminifera from the eastern Indian Ocean. In Davies, T. A., Luyendyk, B. P., et al., Init. Repts. DSDP, 26: Washington (U.S. Govt. Printing Office), 745-769.

Hocking, R. M., 1988. Regional geology of the northern Carnarvon Basin. In Purcell, P. G., and Purcell, R. R. (Eds.), The North West Shelf. Australia. Proc. Pet. Expl. Soc., Australia Sym. (Perth, 1988), 97-114.

Home, P. C., Dalton, D. G., and Brannan, J., 1990. Geological evolution of the western Papuan Basin. In Carman, G. J. and Carman, Z. (Eds.), Petroleum Exploration in Papua New Guinea. Proc. 1st PNG Pet. Conv. (Port Moresby), 107-117.

Koutsoukos, E. A., Leary, P. N., and Hart, M. B., 1989. Favusella Michael (1972): Evidence of ecophenotypic adaptation of a planktonic foraminifer to shallow-water carbonate environments during the mid-Cretaceous. J. Foramin. Res., 19:324-336.

Krasheninnikov, V. A., 1974a. Cretaceous and Paleogene planktonic foraminifera, Leg 27 of the Deep Sea Drilling Project. In Veevers, J. J., Heirtzler, J. R., et al., Init. Repts. DSDP, 27: Washington (U.S. Govt. Printing Office), 663-671.

1974b. Upper Cretaceous benthonic agglutinated foraminifera Leg 27. In Veevers, J. J., Heirtzler, J. R., et al., Init. Repts. DSDP, 27: Washington (U.S. Govt. Printing Office), 631-661.

Leckie, R. M., 1984. Mid-Cretaceous planktonic foraminiferal biostratigraphy off central Morocco, Deep Sea Drilling Project Leg 79, Sites 545 and 547. In Hinz, K., Winterer, E. L., et al., Init. Repts. DSDP, 79: Washington (U.S. Govt. Printing Office), 579-620.

Loeblich, A. R., and Tappan, H., 1946. New Washita foraminifera. $J$. Paleontol., 20:238-258.

1988. Foraminiferal Genera and Their Classification: New York (Van Nostrand Reinhold).

Longoria, J. F., 1974. Stratigraphic, morphologic and taxonomic studies of Aptian planktonic foraminifera. Rev. Española Micropaleontol., Num. Extra.

Ludbrook, N. H., 1966. Cretaceous biostratigraphy of the Great Artesian Basin in South Australia. Bull. Geol. Surv. South Australia, 40.

1978. Australia. In Moullade, M., and Nairn, A.E.M. (Eds.), The Phanerozoic Geology of the World II. The Mesozoic A: Amsterdam (Elsevier), 209-249.
Ludden, J. N., Gradstein, F. M., et al., 1990. Proc. ODP, Init. Repts., 123: College Station, TX (Ocean Drilling Program).

Magné, J., and Sigal, J., 1954. Description des espèces nouvelles, 1 Foraminifères. Bull. Soc. Géol. France, 3:480-489.

Magniez, F., and Sigal, J., 1985. Barremian and Albian foraminifera, Site 549, Leg. 80. In Graciansky, P. C. de, Poag, C. W., et al., Init. Repts. DSDP, 80: Washington (U.S. Govt. Printing Office), 601-628.

Malapris, M., 1965. Les Gavelinellidae et formes affines du gisement Albien de Courcelles (Aube). Rev. Micropaléontol., 8:131-150.

Malapris-Bizouard, M., 1967. Les lingulogavelinelles de l'Albien Inférieur et moyen de l'Aube. Rev. Micropaléontol., 10:128-150.

Michael, F. Y., 1972. Planktonic foraminifera from the Comanchean Series (Cretaceous) of Texas. J. Foramin. Res., 24:200-220.

Miles, G. A. and Orr, W. N., 1980. Planktonic foraminifers from the Bermuda Rise, Deep Sea Drilling Project Legs 51, 52, and 53. In Donnelly, T., Francheteau, J., Bryan, W., Robinson, P., Flower, M., Salisbury, M., et al., Init. Repts. DSDP, 51, 52, 53(Pt. 2): Washington (U.S. Govt. Printing Office), 791-813.

Morozova, V. G., 1948. Foraminifery Nizhnemelovykh otlozheniy rayona G. Sochi (yugo-zapadnyy Kavkaz). Byull. Moskov. Obsh. Ispy. Priridy, Otdel Geol., 23:23-43.

Morrow, A. L., 1934. Foraminifera and ostracoda from the Upper Cretaceous of Kansas. J. Paleontol., 8:186-205.

Moullade, M., 1984. Intérêt des petits foraminifères benthiques "profonds" pour la biostratigraphie et l'analyse des paléoenvironnements océaniques mésozoïques. In Oertli, H. J. (Ed.), Benthos '83, 2nd Int. Symp. Benthic Foramin. (Pau, April 1983): Pau and Bordeaux (Elf Aquitaine, Esso REP, and Total CFP), 429-464.

Narayanan, V., and Scheibnerová, V., 1975. Lingulogavelinella and Orithostella (Foraminifera) from the Utatur Group of the Trichinopoly Cretaceous, south (peninsular) India. Rev. Española Micropaleontol., 7:25-36.

Orbigny, A. d', 1840. Mémoire sur les foraminifères de la craie blanche du bassin du Paris. Mém. Soc. Géol. France, 4:1-51.

Playford, G., Haig, D. W., and Dettmann, M. E., 1975. A mid-Cretaceous microfossil assemblage from the Great Artesian Basin, northwestern Queensland. Neues Jahr. Geol. Paläontol. Abh., 149:333-362.

Plummer, H. J., 1931. Some Cretaceous foraminifera in Texas. Bull. Univ. Texas Bur. Econ. Geol. Technol., 3101:109-203.

Price, R. J., 1975. Palaeoenvironmental interpretations in the Albian of western and southern Europe, as shown by the distribution of selected foraminifera. In Schafer, C. T., and Pelletier, B. R. (Eds.), Proc. Ist Int. Symp. on Benthic Foraminifera of Continental Margins, Part B. Paleoecol. Biostratigr. Maritime Sediments Spec. Publ., 1:625-648.

Quilty, P. G., 1984. Cretaceous foraminiferids from Exmouth Plateau and Kerguelen Ridge, Indian Ocean. Alcheringa, 8:225-241.

Renz, O., 1936. Stratigraphische und mikropalaeontologische Untersushung der Scaglia (Obere Kreide - Tertiär) im Zentralen Apennin. Eclo. Geol. Helv., 29:1-149.

Reuss, A. E., 1845. Die Versteinerungen der böhmischen Kreideformation. Erste Abtheilung: Stuttgart (E. Schweizerbart'sche Verlagsbuchhandlung und Druckerei).

1860. Die Foraminiferen der Westphälischen Kreideformation. Sitzungsberichte der Kaiserlichen Akademie der Wissenschaften in Wien, Math.-Natur. Classe, 40:147-238.

1863. Die Foraminiferen des norddeutschen Hils und Gault. Sitzungsberichte der Kaiserlichen Akademie der Wissenschaften in Wien, Math.-Natur. Classe, 46(1):5-100.

Roemer, F. A., 1841. Die Versteinerungen des norddeutschen Kreidegebirges: Hannover (Hahn).

Schacko, G., 1897. Beitrag über Foraminiferen aus der Cenoman-Kreide von Moltzow in Mecklenburg. Archiv Vere. Freu. Natur. Mecklenburg (1896), 50:161-168.

Scheibnerová, V., 1970. Some notes on palaeoecology and palaeogeography of the Great Artesian Basin, Australia, during the Cretaceous. Search, 1:125-126.

1971a. Lingulogavelinella (Foraminifera) in the Cretaceous of the Great Artesian Basin, Australia. Micropaleontology, 17:109-116. 1971b. Bilingulogavelinella australis n. gen. n. sp. (Foraminifera) from the Cretaceous of the Great Artesian Basin, Australia. $J$. Foramin. Res., 1:122-125. 
1972. Some interesting foraminifera from the Cretaceous of the Great Artesian Basin, Australia. Micropaleontology, 10:212-222.

1974a. The ecology of Scutuloris and other important genera from the Early Cretaceous of the Great Artesian Basin (Australia). Rev. Española Micropaleontol., 6:229-255.

1974b. Aptian-Albian benthonic foraminifera from DSDP Leg 27, Sites 259, 260, and 263, eastern Indian Ocean. In Veevers, J. J., Heirtzler, J. R., et al., Init. Repts. DSDP, 27: Washington (U.S. Govt. Printing Office), 697-741.

1976. Cretaceous foraminifera of the Great Australian Basin.

Mem. Geol. Surv. New South Wales, 17.

1978a. Some Cretaceous foraminifera from Leg 26 of the DSDP in the Indian Ocean. Bull. BMR Geol. Geophys., Australia, 192:137163.

1978b. Aptian and Albian benthic foraminifers of Leg 40, Sites 363 and 364, southern Atlantic. In Bolli, H. M., Ryan, W.B.F., et al., Init. Repts. DSDP, 40: Washington (U.S. Govt. Printing Office), 741-757.

1986. Cretaceous biostratigraphy of the Great Australian Basin based on benthonic foraminifera. Rec. New South Wales Geol. Surv., 22:1-98.

Sigal, J., 1979. Chronostratigraphy and ecostratigraphy of Cretaceous formations. In Ryan, W.B.F., Sibuet, J.-C., et al., Init. Repts. DSDP, 47(2): Washington (U.S. Govt. Printing Office), 287-326.

Sliter, W. V., 1977. Cretaceous benthic foraminifers from the western south Atlantic Leg 39, Deep Sea Drilling Project. In Barker, P. F., Dalziel, I.W.D., et al., Init. Repts. DSDP, 36: Washington (U.S. Govt. Printing Office), 519-545.

1986. Cretaceous redeposited benthic foraminifers from Deep Sea Drilling Project Site 585 in the East Mariana Basin, western equatorial Pacific, and implications for the geologic history of the region. In Moberly, R., Schlanger, S. O., et al., Init. Repts. DSDP, 89: Washington (U.S. Govt. Printing Office), 327-361.

Takayanagi, Y., and Iwamoto, H., 1962. Cretaceous planktonic foraminifera from the Middle Yezo group of the Ikushumbetsu, Miruto, and Hatonosu areas, Hokkaido. Trans. Proc. Paleontol. Soc. Japan, n.s., 45:183-196.

Tappan, H., 1940. Foraminifera from the Grayson Formation of northern Texas. J. Paleontol., 14:93-126.

Thierstein, H. R., 1974. Calcareous nannoplankton-Leg 26, Deep Sea Drilling Project. In Davies, T. A., Luyendyk, B. P., et al., Init. Repts. DSDP, 26: Washington (U.S. Govt. Printing Office), 619-668.

Veevers, J. J., 1984. Phanerozoic Earth History of Australia: Oxford (Clarendon Press).

Veevers, J. J., Heirtzler, J. R., et al., 1974. Init. Repts. DSDP, 27, Washington (U.S. Govt. Printing Office).

Veevers, J. J., Powell, C. McA., and Roots, S. R., 1991. Review of seafloor spreading around Australia 1. Synthesis of the patterns of spreading. Australian J. Earth Sci., 38:373-389.

Wonders, A.A.H., 1975. Cretaceous planktonic foraminifera of the Planomalina buxtorfi group from El Burrueco, southern Spain. Proc. Koninklijke Neder. Akad. Wetenschappen (Amsterdam), Ser. B, 78:83-93.

1980. Middle and Late Cretaceous planktonic foraminifera of the western Mediterranean area. Utrecht Micropaleontol. Bull., 24.

Date of initial receipt: 15 June 1990

Date of acceptance: 30 October 1990

Ms 123B-122

\section{APPENDIX \\ Taxonomic List}

Foraminifers recorded from Site 766 are classified here under the Orders recognized by Haynes (1981), and the families and genera diagnosed by Loeblich and Tappan (1988). For each named species, the original nomenclature is cited (following Ellis and Messina, 1945, et seq.), and for several species, references to significant taxonomic revisions are given. Published illustrated records of the species from confirmed Aptian-Albian deposits in the Australian region also are listed.

\author{
Order ASTRORHIZIDA \\ Family AMMODISCIDAE \\ Genus GLOMOSPIRELLA \\ ?Glomospirella sp.
}

Glomospira gordialis Parker and Jones; Scheibnerová, 1974b, PI. 1, Fig. 3. Perth Abyssal Plain, Albian.

Genus REPMANIA

Repmania corona (Cushman and Jarvis, 1928)

Glomospira charoides (Jones and Parker) var. corona Cushman and Jarvis, 1928, p. 89, Pl. 12, Figs. 9-11.

\section{Order LITUOLIDA \\ Family AMMOSPHAEROIDINIDAE Genus AMMOSPHAEROIDINA \\ Ammosphaeroidina sp. \\ Family SPIROPLECTAMMINIDAE Genus HETERANTYX \\ Heterantyx cf. cretosa (ten Dam, 1950)}

cf. Spiroplectammina rectangularis ten Dam var. cretosa ten Dam, 1950, p. 11, Pl. 1, Fig. 9.

?Spirobolivina australis Scheibnerová, 1974b, p. 713, Pl. 4, Fig. 4, Pl. 9 , Fig. 14. Perth Abyssal Plain, Albian.

\section{Genus SPIROPLECTINELLA}

Spiroplectinella cf. gandolfii (Carbonnier, 1952)

cf. Spiroplectammina gandolfii Carbonnier, 1952, p. 112, PI. 5, Figs. $2 \mathrm{a}-2 \mathrm{~b}$.

Textularia sp. B; Haig, 1981, Pl. 1, Figs. 22-23. Papuan Basin, upper Albian.

Family VERNEUILINIDAE Genus GAUDRYINA

Gaudryina aff. richteri Grabert, 1959

aff. Gaudryina richteri Grabert, 1959, p. 12, Pl. 1, Figs 1a-2c, Pl. 3, Figs. 46-47.

$$
\begin{gathered}
\text { Genus PSEUDOGAUDRYINELLA } \\
\text { Pseudogaudryinella } \mathrm{sp} . \mathrm{A}
\end{gathered}
$$

Migros sp. ; Scheibnerová, 1974b, p. 710, Pl. 1, Fig. 24, Pl. 9, Fig. 13; Perth and Gascoyne abyssal plains, Albian.

$$
\begin{gathered}
\text { Pseudogaudryinella sp. B } \\
\text { Pseudogaudryinella sp. C } \\
\text { Family TRITAXIIDAE } \\
\text { Genus TRITAXIA } \\
\text { Tritaxia gaultina (Morozova, 1948) }
\end{gathered}
$$

Clavulina gaultina Morozova, 1948, p. 36, P1. 1, Fig. 4.

Clavulina gabonica Le Calvez et al.; Scheibnerová, 1974b, p. 711. Pl. 2, Figs. 5-8, 9; Perth Abyssal Plain, Albian.

$$
\begin{gathered}
\text { Family GLOBOTEXTULARIIDAE } \\
\text { Genus REMESELLA } \\
\text { Remesella } \mathrm{sp} .
\end{gathered}
$$

Matanzia sp., Scheibnerová, 1974b, p. 711, Pl. 9, Figs. 15a, 15b. Perth Abyssal Plain, Albian.

?Dorothia sp., Scheibnerová, 1974b, p. 710, Pl. 2, Figs. 1-3. Perth Abyssal Plain, Albian.

?Eggerella sp., Scheibnerová, 1974b, p. 710, Pl. 2, Fig. 4. Perth Abyssal Plain, Albian. 


\author{
Family EGGERELLIDAE \\ Genus DOROTHIA \\ Dorothia sp.
}

Genus MARSSONELLA

Marssonella oxycona (Reuss, 1860)

Gaudryina oxycona Reuss, 1860, p. 229, Pl. 12, Figs. 3a-3c.

Marssonella oxycona (Reuss); Haig, 1980, p. 126, PI. 8, Fig. 19; northern Carpentaria Basin, upper Albian.

Marssonella oxycona (Reuss); Haig, 1981, Pl. 1, Fig. 32; Papuan Basin, Albian-Cenomanian.

\section{Order NODOSARIIDA \\ Family ICHTHYOLARIIDAE \\ Genus LINGULONDOSARIA \\ Lingulonodosaria loryi (Berthelin)}

Frondicularia loryi Berthelin 1880, p. 60, Pl. 4, Figs. 5a, 5 b.

Lingulina loryi (Berthelin); Haig, 1982, p. 18, Pl. 3, Fig. 17; Laura Basin and northern Carpentaria Basin, Albian.

$$
\text { Lingulonodosaria nodosaria (Reuss) }
$$

Lingulina nodosaria Reuss, 1863, p. 59, Pl. 5, Figs. 12a, 12 b.

Lingulina nodosaria Reuss; Haig, 1981, Pl. 2, Fig. 27; Papuan Basin, Albian-cenomanian.

Lingulina nodosaria Reuss; Haig, 1982, p. 19, Pl. 3, Figs. 19, 20; "Great Artesian Basin," Aptian-Albian (see for other Australian records).

$$
\begin{aligned}
& \text { Lingulonodosaria sp. A } \\
& \text { Lingulonodosaria sp. B }
\end{aligned}
$$

Family NODOSARIIDAE Genus FRONDICULARIA

?Frondicularia sp.

Genus GRIGELIS

?Grigelis sp.

Genus LAEVIDENTALINA

Laevidentalina cylindroides (Reuss, 1860)

Dentalina cylindroides Reuss, 1860 , p. 185 , Pl. 1, Fig. 8.

Dentalina cylindroides Reuss; Haig, 1981, P1. 2, Fig. 11; Papuan Basin, upper Albian-Cenomanian.

Dentalina cylindroides Reuss; Haig, 1982, p. 6, Pl. 2, Figs. 19, 20; Laura and northern Carpentaria basins, Albian.

\section{Laevidentalina gracilis (d'Orbigny, 1840)}

Nodosaria (Dentalina) gracilis d'Orbigny, 1840, p. 14, PI. 1, Fig. 5.

Dentalina gracilis Orbigny; Haig, 1982, p. 7, Pl. 1, Figs. 11.12; Laura Basin, Albian.

Laevidentalina nana (Reuss, 1863)

Nodosaria (Dentalina) nana Reuss, 1863, p. 39, Pl. 2, Figs. 10,18.

$$
\text { Laevidentalina oligostegia (Reuss, 1845) }
$$

Nodosaria (Dentalina) oligostegia Reuss, 1845, p. 27, Pl. 13, Figs. 19,20.

Dentalina oligostegia Reuss; Haig, 1982, p. 8, Pl. 1, Figs. 8-10; Laura, northern Carpentaria, and northern Eromanga basins, Albian (see for other Australian records).

\section{Genus LINGULINA}

Lingulina buddencanyonensis Dailey, 1970

Lingulina buddencanyonensis Dailey, 1970, p. 106, P1. 12, Fig. 3.

Lingulina buddencanyonensis Dailey; Haig, 1982, p. 17, PI. 3, Fig. 18; "Great Artesian Basin," Aptian-Albian.

Lingulina sp.
Genus PSEUDONODOSARIA

Pseudonodosaria humilis (Roemer, 1841)

Nodosaria humilis Roemer, 1841, p. 95, Pl. 15, Fig. 6.

Pseudonodosaria humilis (Roemer); Haig, 1981, Pl. 2, Fig. 16; Papuan Basin, upper Albian-Cenomanian.

Pseudonodosaria humilis (Roemer); Haig, 1982, p. 15,16, PI. 3, Figs, 4,5 "Great Artesian Basin," Aptian-Albian (see for other Australian records).

\section{Genus PYRAMIDULINA}

Pyramidulina obscura (Reuss, 1845)

Nodosaria (Nodosaria) obscura Reuss, 1845, p. 26, P1. 13, Figs. 7-9.

Nodosaria obscura Reuss; Haig, 1981, Pl. 2, Fig. 8; Papuan Basin, upper Albian-Cenomanian.

Nodosaria obscura Reuss; Haig, 1982, p. 13, Pl. 2, Figs. 39-43; "Great Artesian Basin," Aptian-Albian (see for other Australian records).

Pyramidulina sceptrum (Reuss, 1863)

Nodosaria (Nodosaria) sceptrum Reuss, p. 37, Pl. 2, Figs. 3a, 3b.

Nodosaria sceptrum Reuss; Haig, 1981, Pl. 2, Fig. 7; Papuan Basin, upper Albian-Cenomanian.

Nodosaria sceptrum Reuss; Haig, 1982, p. 14, Pl. 2, Figs. 29-32; "Great Artesian Basin," Albian (see for other Australian records).

$$
\text { Pyramidulina sp. }
$$

Genus SVENIA

Svenia cf. hamiltonensis (Ludbrook, 1966)

cf. Dentalina hamiltonensis Ludbrook, 1966, p. 112,113, Pl. 8, Fig. 5; Eromanga Basin, Aptian-Albian (see other records from Australia listed by Haig, 1982 , p. 7).

\section{Family VAGINULINIDAE Genus ASTACOLUS Astacolus cf. scitula (Berthelin, 1880)}

cf. Cristellaria scitula Berthelin, 1880, p. 54, P1. 3, Figs. 3a-3c. cf. Astacolus scitula (Berthelin); Haig, 1982, p. 20, PI. 4, Figs. 5-7. Laura and northern Carpentaria basins, Albian.

$$
\begin{gathered}
\text { Astacolus sp. A } \\
\text { Astacolus spp. }
\end{gathered}
$$

\section{Genus CITHARINA}

Citharina cf. petila Eicher and Worstell, 1970

cf. Citharina petila Eicher and Worstell, 1970, p. 284, Pl. 2, Figs. 20,21. cf. Citharina petila Eicher and Worstell; Haig, 1982, p. 30,31, Pl. 6, Figs. 7-10; "Great Artesian Basin," Albian.

\section{Citharina sp. A}

cf. Citharina sp., Haig, 1981, Pl. 2, Fig. 29; Papuan Basin, upper AlbianCenomanian.

\section{Citharina sp. B}

Citharina sp. B of Haig, 1982, p. 33, Pl. 6, Figs. 18,19; Laura and northern Carpentaria basins, Albian.

\section{Genus HEMIROBULINA}

Hemirobulina hamulus (Chapman, 1894)

Marginulina hamulus Chapman, 1894, p. 161, Pl. 4, Figs. 13a, 13b.

Marginulina hamulus Chapman; Haig, 1981, Pl. 2, Fig. 20; Papuan Basin, upper Albian-Cenomanian.

Marginulina hamulus Chapman; Haig, 1982, p. 25, P1. 5, Figs. 7,8; "Grea Artesian Basin," Aptian-Albian (see for other Australian records). 


\section{Genus LENTICULINA}

Lenticulina spp.

Genus PLANULARIA

Planularia bradyana (Chapman, 1894)

Cristellaria bradyana Chapman, 1894, p. 654, Pl.10, Figs. 13a, 13b.

Planularia bradyana (Chapman); Haig, 1981, Pl. 2, Fig. 13; Papuan Basin, upper Albian-Cenomanian.

Planularia bradyana (Chapman); Haig, 1982, p. 21, Pl. 4, Figs. 8-11; northern Carpentaria Basin, upper Albian (see for other Australian records).

Genus PSILOCITHARELLA

Psilocitharella recta (Reuss, 1863)

Vaginulina recta Reuss, 1863, p. 48, Pl. 3, Figs. 14-15b.

Citharina recta (Reuss); Haig, 1982, p. 31,32, PI. 6, Figs. 31-34; Laura, northern Carpentaria, and northern Eromanga basins, Albian.

Genus SARACENARIA

Saracenaria spinosa Eichenberg, 1935

Saracenaria spinosa Eichenberg, 1935, p. 10, Pl. 4, Figs. 5a-5c.

Saracenaria triangularis (d'Orbigny, 1840)

Cristellaria triangularis d'Orbigny, 1840, p. 27, PI. 2, Figs. 21-22.

\section{Genus VAGINULINA}

Vaginulina sp.

\section{Genus VAGINULINOPSIS}

Vaginulinopsis sp.

\section{Family POLYMORPHINIDAE \\ Genus GLOBULINA}

Globulina prisca Reuss, 1863

Polymorphina (Globulina) prisca Reuss, 1863, p. 79, Pl. 9, Figs. 8a, 8b. Globulina lacrima Reuss; Haig, 1981, Pl. 2, Fig. 25; Papuan Basin, upper Albian-Cenomanian.

Globulina prisca Reuss; Haig, 1982, p. 35, Pl. 7, Fig. 7; Laura, northern Carpentaria, and northern Eromanga basins, Albian.

\section{Genus RAMULINA}

Ramulina spp.

Family GLANDULINIDAE

Genus TRICARINELLA

Tricarinella excavata (Reuss, 1863)

Rhabdogonium excavatum Reuss, 1863, p. 91, Pl. 12, Figs. 8a-8c.

Tristix excavata (Reuss); Haig, 1981, Pl. 2, Fig. 26; Papuan Basin, upper Albian-Cenomanian.

Tristix excavata (Reuss); Haig, 1982, p. 38, Pl. 7, Figs. 16,17; "Great Artesian Basin," Albian (see for other Australian records).

\section{Order BULIMINIDA \\ Family BOLIVINIDAE \\ Genus TAPPANINA \\ Tappanina sp. \\ Family TURRILINIDAE \\ Genus CUNEUS \\ Cuneus cf. ludbrookae (Haig, 1982)}

cf. Neobulimina ludbrookae Haig, 1982, p. 50-52, P1. 10, Figs. 1-7, P1. 16, Fig. 4; Carpentaria Basin, upper Albian.

\section{Genus EURYCHEILOSTOMA}

?Eurycheilostoma $\mathrm{cf}$. hergottensis (Ludbrook, 1966)

cf. Praebulimina hergottensis Ludbrook, 1966, p. 132,133, Pl. 10, Fig. 21; Eromanga Basin, Aptian.
?Neobulimina australiana Ludbrook; Scheibnerová, 1974c, p. 712, Pl. 4, Figs. 1,2; Pl. 10, Figs. 10,11; Perth Abyssal Plain, Albian (not Neobulimina australiana Lubrook = Neobulimina albertensis of Haig, 1982, p. 48, Pl. 9, Figs. 10-20).

cf. Eurycheilostoma hergottensis (Ludbrook); Haig, 1982, p. 47, PI. 10, Figs. 11,12; Pl. 11, Figs. 1-3, Pl. 16, Fig. 3; "Great Artesian Basin," Aptian.

\section{Genus PRAEBULIMINA}

Praebulimina cf. nannina (Tappan, 1940)

cf. Bulimina nannina Tappan, 1940, p. 116, P1. 19, Figs. 4a, 4b.

Praebulimina sp., Haig, 1981, Pl. 3, Figs. 3,4; Papuan Basin, AlbianCenomanian.

\author{
Family FURSENKOINIDAE \\ Genus CORYPHOSTOMA \\ Coryphostoma sp.
}

Coryphostoma sp. A, Haig, 1982, p. 62, 63, Pl. 12, Figs. 19-22; Laura, northern Carpentaria, and northern Eromanga basins, Albian (see for other Australian records)

\section{Family PLEUROSTOMELLIDAE Genus ELLIPSOGLANDULINA Ellipsoglandulina sp.}

\section{Genus ELLIPSODELLA}

Ellipsoidella cf. cuneata (Loeblich and Tappan, 1946)

cf. Nodosarella cuneata Loeblich and Tappan, 1946, p. 255, PI. 37, Figs. 9a,9b.

Ellipsoidella sp. cf. E. cuneata (Loeblich and Tappan); Haig, 1982, p. 59 , Pl. 12, Figs. 9-11; northern Carpentaria Basin, upper Albian.

Genus PLEUROSTOMELLA

Pleurostomella aff. reussi Berthelin, 1880

aff. Pleurostomella reussi Berthelin, 1880, p. 28, Pl. 1, Figs. 10-12.

aff. Pleurostomella reussi Berthelin; Haig, 1981, P1. 3, Fig. 8; Papuan Basin, Albian.

aff. Pleurostomella reussi Berthelin; Haig, 1982, p. 59-61, Pl. 12, Figs. 1-8; Laura, northern Carpentaria, and northern Eromanga basins, Albian (see for other Australian records).

Order ROTALIIDA
Family PATELLINIDAE
Genus HERGOTTELLA
Hergottella sp.

Family BAGGINIDAE
Genus SEROVAINA
Serovaina gracillima (ten Dam, 1947)

Valvulineria gracillima ten Dam, 1947, p. 27, Text-figs. 4a-4c.

Valvulineria sp. A, Haig, 1982 , p. 58 , Pl. 12, Figs. 31,32 ; northern Carpentaria Basin, upper Albian.

?Valvulineria loetterlei (Tappan); Quilty, 1984, p. 233, Fig. 4A; Exmouth Plateau, upper Albian.

Serovaina infracretacea (Morozova, 1948)

Gyroidina nitida Reuss var. infracretacea Morozova, 1948, p. 40, Pl. 2, Figs. 12-14.

Gyroidinoides cf. primitiva Hofker; Scheibnerová, 1974b, p. 714, Pl. 5 , Figs. 10-12, Pl. 11, Figs. 6a-6c; Perth and Gascoyne Abyssal plains, Albian.

Valvulineria loetterlei Tappan; Scheibnerová,1978a, p. 138, Pl. 3, Figs. 5,6; Naturaliste Plateau, Albian.

Valvulineria crespinae Ludbrook; Haig, 1981, Pl. 3, Figs. 10,11; Papuan Basin, upper Albian-Cenomanian. 
Family QUADRIMORPHINIDAE Genus QUADRIMORPHINA

Quadrimorphina allomorphinoides (Reuss, 1860)

Valvulina allomorphinoides Reuss, 1860, p. 223, PI. X, Figs. XX.

Conorboides minutissima (Tappan); Haig, 1981, Pl. 2, Figs. 32-33; Papuan Basin, upper Albian-Cenomanian.

Conorboides minutissima (Tappan); Haig, 1982, p. 43, Pl. 9, Figs. 6-9; Laura and northern Carpentaria basins, Albian (see for other Australian records).

\section{Family GLOBOROTALITIDAE Genus GLOBOROTALITES}

Globorotalites aptiensis Bettenstaedt, 1952

Globorotalites bartensteini Bettenstaedt subsp. aptiensis Bettenstaedt, 1952, p. 282, 278, Pl. 3, Figs. 32a-32c, Pl. 4, Figs. 59-72.

Globorotalites sp., Haig, 1982, Pl. 3, Figs. 14,15; Papuan Basin, (?)upper Albian or Cenomanian.

\section{Family OSANGULARIIDAE} Genus CHARLTONINA

Charltonina cf. australis Scheibnerová, 1978

cf. Charltonina australis Scheibnerová, 1978a, p. 140, pl. 5, Figs. 2-5.

Genus OSANGULARIA

Osangularia schloenbachi (Reuss, 1863)

Rotalia schloenbachi Reuss, 1863, p. 84 , Pl. 10, Fig. 5 (revised as Osangularia schloenbachi by Crittenden, 1983, p. 40-64).

Osangularia utaturensis (Sastry and Sastri); Scheibnerová, 1974b, p. 714 Pl. 4, Figs. 27,28; Pl. 5, Figs. 1-9; Pl. 11, Figs. 4a-5c; Perth and Gascoyne abyssal plains, Albian.

Osangularia utaturensis (Sastry and Sastri); Haig, 1981, PI. 3, Figs. 18,19; Papuan Basin, upper Albian-Cenomanian.

Osangularia sp. A, Haig, 1982, p. 64, Pl. 13, Figs. 16,17; Laura Basin, Albian.

\section{Family GAVELINELLIDAE Genus BERTHELINA Berthelina intermedia (Berthelin, 1880)}

Anomalina intermedia Berthelin, 1880, p. 67, Pl. 4, Figs. 14a, 14b. Anomalinoides intermedia (Berthelin); Haig, 1981, Pl. 3, Fig. 21; Papuan Basin, upper Albian-Cenomanian.

Anomalinoides intermedia (Berthelin); Haig, 1982, p. 66,67, Pl. 13, Figs. 1-3; Laura, northern Carpentaria, and eastern Eromanga basins, Albian (see for other Australian records).

Genus GAVELINELLA

Gavelinella cf. andersoni (Church, 1968)

cf. Planulina andersoni Church, 1968, p. 567, Pl. 8, Figs. 7a-7c.

Planulina sp., Haig, 1981, Pl. 3, Figs. 12,13; Papuan Basin, upper Albian.

Gavelinella cf. indica (Scheibnerová, 1974)

cf. Orithostella indica Scheibnerová, 1974b, p. 715, PI. 7, Figs. 4, 8-13; PI. 8, Figs. 1-9; PI. 11, Figs. 9a-9c; Perth Abyssal Plain, Albian.

Lingulogavelinella indica (Scheibnerová); Haig, 1982, p. 74, 75, Pl. 13, Figs. 18-20; Laura and northern Carpentaria basins, upper part of lower Albian-lower part of middle Albian.

\section{Gavelinella sp. A}

?Planulina sp., Quilty, 1984, p. 236-237, Figs, 6A, 6B; Exmouth Plateau, upper Albian.

\section{Gavelinella sp. B}

Orithostella sp. A, Haig, 1982, p. 75, Pl. 3, Figs. 9-11; Laura and northern Carpentaria basins, Albian.
Genus SCHEIBNEROVA

Scheibnerova protindica Quilty, 1984

?Globotruncana sp., Scheibnerová, 1978a, Pl. 7, Figs. 10-12; Naturaliste Plateau, upper Albian.

Scheibnerova protindica Quilty, 1984, p. 234, Figs. 5A-5K; Exmouth Plateau, upper Albian.

$$
\begin{gathered}
\text { Order GLOBIGERINIDA } \\
\text { Family GLOBIGERINELLOIDIDAE } \\
\text { Genus GLOBIGERINELLOIDES } \\
\text { Globigerinelloides aptiense Longoria, } 1974
\end{gathered}
$$

Globigerinelloides aptiense Longoria, 1974, p. 79,80, Pl. 4, Figs. 9,10; Pl. 8, Figs. 4-6, 17, 18.

Globigerinelloides cf. aptiense Longoria, 1974

cf. Globigerinelloides aptiense Longoria, 1974, p. 79,80, Pl. 4, Figs. 9,10; Pl. 8, Figs. 4-6, 17,18

Globigerinelloides $\mathrm{cf}$. bentonensis (Morrow, 1934)

Anomalina bentonensis Morrow, 1934, p. 201, Pl. 30, Fig. 4

Globigerinelloides bentonensis (Morrow); Krasheninnikov, 1974a, p. 665,666 , Pl. 2, Figs. 12-13; Gascoyne Abyssal Plain, Albian.

\section{Family PLANOMALINIDAE \\ Genus PLANOMALINA}

Planomalina praebuxtorfi Wonders, 1975

Planomalina praebuxtorfi Wonders, 1975, p. 90,91, Pl. 1, Figs. 1a-2c; Text-fig. 4, Figs. 2a, 2b.

Planomalina buxtorfi (Gandolfi, 1942)

Planulina buxtorfi Gandolfi, 1942, p. 103, Pl. 3, Figs. 7a-7c (emended by Wonders, 1975 , p. 91,92).

Planomalina buxtorfi (Gandolfi); Haig, 1981, Pl. 3, Figs. 26,32; Papuan Basin, upper Albian.

Planomalina buxtorfi (Gandolfi); Quilty, 1984, p. 231,232, Fig. 3A Exmouth Plateau, upper Albian.

Planomalina buxtorfi (Gandolfi); Belford, 1985, p. 185, Figs. 3, 4-11; Papuan Basin, upper Albian.

\author{
Family SCHACKOINIDAE \\ Genus SCHACKOINA \\ Schackoina cenomana (Schacko, 1897)
}

Siderolina cenomana Schacko, 1897, p. 166, Pl. 4, Figs. 3-5.

Schackoina cenomana (Schacko); Playford et al., 1975, Fig. 2, Nos. 1-4; Carpentaria Basin, upper Albian.

\section{Family HEDBERGELLIDAE \\ Genus CLAVIHEDBERGELLA}

Clavihedbergella simplicissima (Magné and Sigal, 1954)

Hastigerinella simplicissima Magné and Sigal, 1954, p. 487, Pl. 14, Figs. $11 \mathrm{a}-11 \mathrm{c}$.

Hedbergella simplicissima Magné and Sigal; Herb, 1974, p. 752,753, PI. 3, Figs. 9-13; Naturaliste Plateau, upper Albian.

Clavihedbergella simplicissima (Magné and Sigal); Quilty, 1984, p. 233, Fig. 3C; Exmouth Plateau, upper Albian.

Hedbergella simplicissima (Magné and Sigal); Belford, 1985, p. 185, Fig. 2, Nos. 28-33.

\section{Clavihedbergella $\mathrm{sp}$}

Hedbergella amabilis Loeblich and Tappan; Playford et al., 1975, Fig. 3 , Nos. 7-9; northern Carpentaria Basin, upper Albian (smooth walled not $H$. amabilis Loeblich and Tappan, $1961=$ Hastigerinella simplicissima Magné and Sigal, 1954) 
Genus HEDBERGELLA

Hedbergella sp. cf. Blefuscuiana aptica (Agalarova in Dzhafarov et al., 1951)

cf. Globigerina aptica Agalarova in Dzhafarov et al., 1951, p. 49, Pl. 8, Figs. 9-11.

cf. Blefusciana aptica (Agalarova); Banner and Desai, 1988, p. 160, Pl. 5, Figs. 4-7.

Blefuscuiana cf. aptica (Agalarova); Banner and Desai, 1988, Pl. 3, Figs. 4-5.

Hedbergella planispira (Tappan, 1940)

Globigerina planispira Tappan, 1940, p. 122, PI. 19, Figs. 12a-12c (see also illustrations of topotype by Loeblich and Tappan, 1988, PI. 495, Figs. 13-15; and scanning electron micrographs of Banner and Desai, 1988, Pl. 3, Figs. 8a-9).

Hedbergella globigerinellinoides (Subbotina); Krasheninnikov, 1974a, p. 665, Pl. 1, Figs. 13-15; Perth Abyssal Plain, Albian.

Hedbergella planispira (Tappan); Playford et al., 1975, Fig. 3, Nos. 10,11; "Great Artesian Basin," lower-middle Albian.

Hedbergella cf. planispira (Tappan, 1940)

cf. Globigerina planispira Tappan, 1940, p. 122, PI. 19, Figs. 12a-12c.

Hedbergella cf. punctata Michael, 1972

cf. Hedbergella(?) punctata Michael, 1972, p. 212, Pl. 3, Figs. 1-3, Pl. 7 , Figs. 1,2

Hedbergella punctata Michael; Playford et al., 1975, Fig. 2, Nos. 6-9; "Great Artesian Basin," upper Albian.

Hedbergella planispira (Tappan); Herb, 1974, p. 752, PI. 3, Figs. 1-8; Naturaliste Plateau, upper Albian.

?Hedhergella planispira (Tappan); Belford, 1985, p. 185, Fig. 2, 22-27; Papuan Basin, upper Albian (not Globigerina planispira Tappan).

Hedbergella trocoidea (Gandolfi, 1942)

Anomalina lorneiana (d'Orbigny) var. trocoidea Gandolfi, 1942, p. 98, Pl. 2, Fig. 1; Pl. 4, Figs. 2,3; Pl. 13, Figs. 2-5.
Hedbergella trocoidea Gandolfi; Krasheninnikov, 1974a, p. 665, Pl. 2, Figs. 4-6; Gascoyne Abyssal Plain, Albian.

Hedbergella yezoana Takayanagi and Iwamoto, 1962

Hedbergella yezoana Takayanagi and Iwamoto, 1962, p. 191,192, Pl. 28, Figs. 1-2.

Hedbergella sp. A, Playford et al., 1975, p. 343,344, Fig.2, Nos. 10-12; Carpentaria Basin, upper Albian.

Hedbergella yezoana Takayanagi and Iwamoto; Miles and Orr, 1980, p. 799 , Pl. 4, Figs. 1-3.

\section{Genus PRAEGLOBOTRUNCANA}

Praeglobotruncana delrioensis (Plummer, 1931)

Globorotalia delrioensis Plummer, 1931, p. 199, Pl. 13, Fig. 2.

Praeglobotruncana delrioensis (Plummer); Herb, 1974, p. 753, Pl. 3. Fig. 14, Text-figs. 5,6; Naturaliste Plateau, upper Albian.

Praeglobotruncana delrioensis (Plummer); Belford, 1985, p. 187, Figs. 2, 34-42; Papuan Basin, upper Albian.

\section{Family ROTALIPORIDAE Genus ROTALIPORA}

Rotalipora appenninica (Renz, 1936)

Globotruncana appenninica Renz, 1936, p. 14, Fig. 2.

Rotalipora appenninica (Renz); Belford, 1985, p. 187, Fig. 3, 12-17; Papuan Basin, upper Albian.

\section{Rotalipora ticinensis (Gandolfi, 1942)}

Globotruncana ticinensis Gandolfi, 1942, p. 113, PI. 2, Fig. 3; PI. 4, Figs. 10,11 .

Rotalipora ticinensis (Gandolfi); Belford, 1985, p. 187, Fig. 3, 18-24; Papuan Basin, upper Albian. 

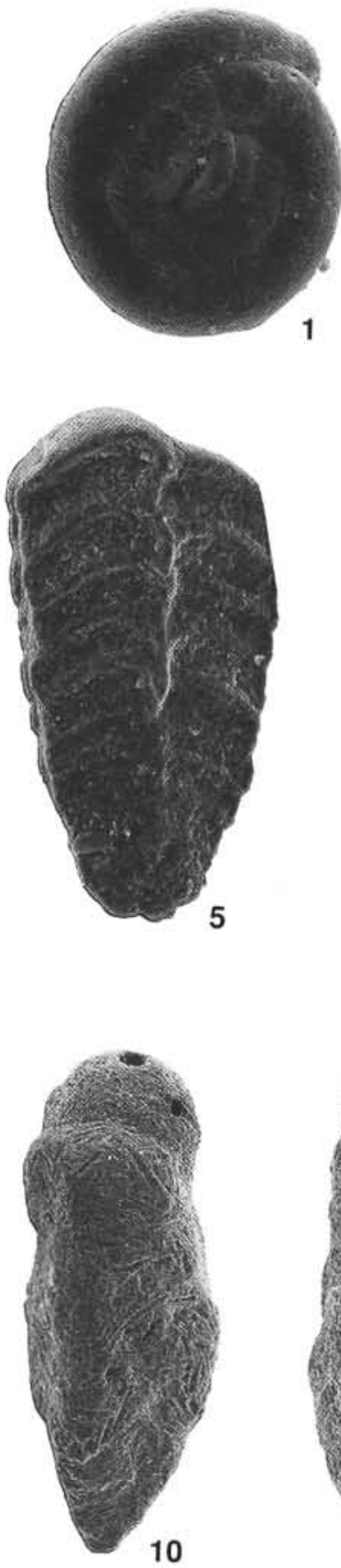

10
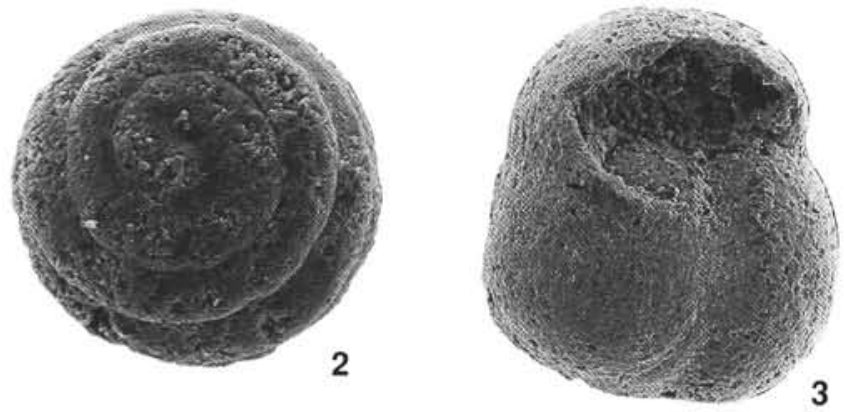
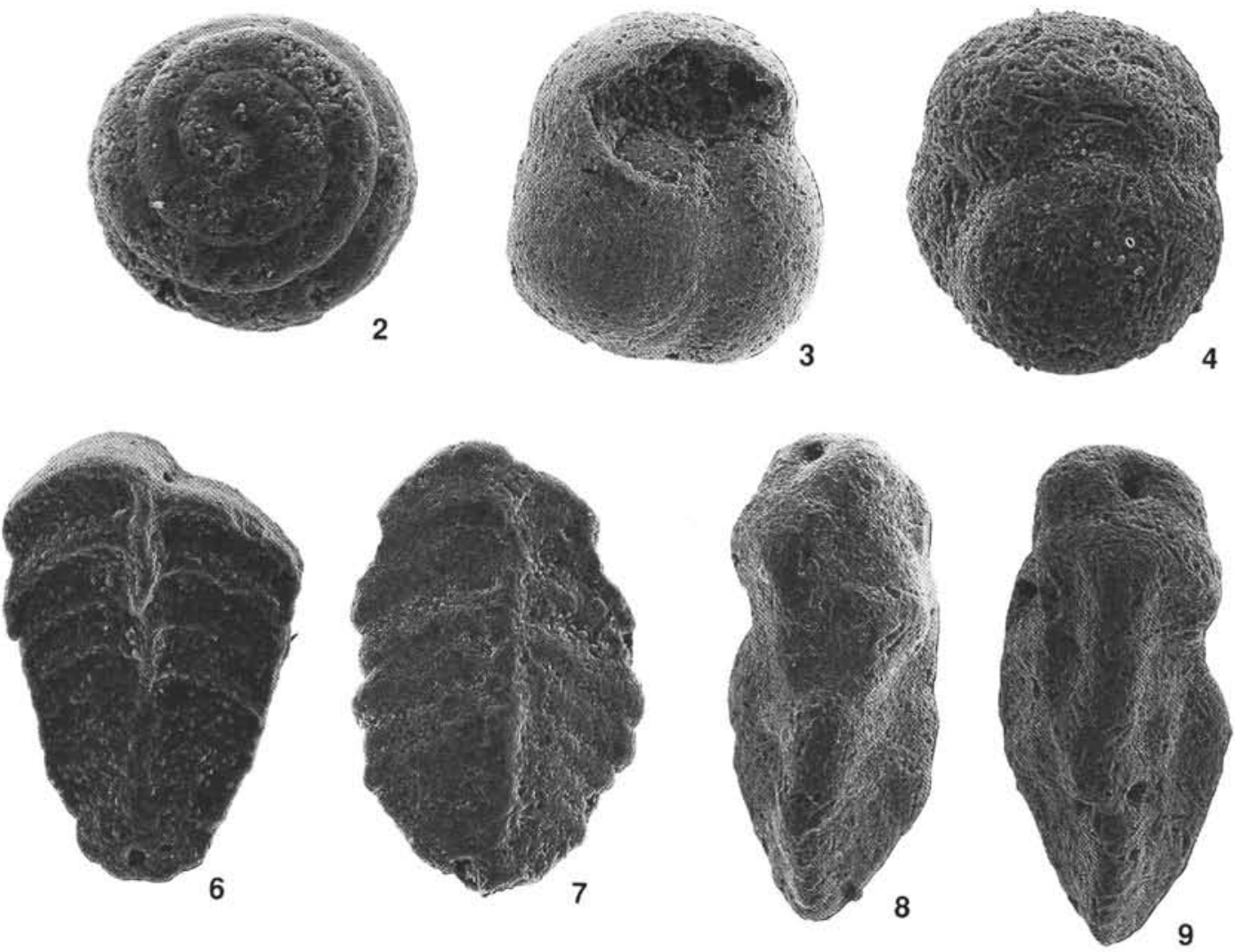

Plate 1. 1. ?Glomospirella sp., Sample 123-766A-19R-CC, $\times 160$. 2. Repmania corona (Cushman and Jarvis), Sample 123-766A-19R-4, 23-27 cm,
$\times 170$. 3,4. Ammosphaeroidina sp., (3) Sample $123-766 \mathrm{~A}-17 \mathrm{R}-5,29-32 \mathrm{~cm} \times 85$, (4) Sample $123-766 \mathrm{~A}-20 \mathrm{R}-\mathrm{CC}, \times 90.5,6$. Heterantyx cf. cretosa (ten Dam), (5) Sample 123-766A-17R-CC, $\times 120$, (6) Sample 123-766A-17R-CC, $\times 135$. 7. Spiroplectinella cf. gandolfii (Carbonnier), Sample 123-766A16R-3, 46-50 cm, $\times 100.8$ 8. Gaudryina aff. richteri Grabert, (8) Sample 123-766A-20R-3, 68-72 cm, $\times 90$, (9) Sample 123-766A-20R-3, 68-72 cm, $\times 80$. 10,11. Pseudogaudryinella sp. A, (10) Sample 123-766A-20R-3, 68-72 cm, $\times 60$, (11) Sample 123-766A-20R-3, 68-72 cm, $\times 50$. 12. Pseudogaudryinella sp. B, Sample 123-766A-17R-5, 29-32 cm, $\times 50$. 13,14. Pseudogaudryinella sp. C, (13) Sample 123-766A-16R-3, 46-50 cm, $\times 100,(14)$ Sample 123-766A-16R-3, 46-50 cm, ×75. 15. Tritaxia gaultina (Morozova), Sample 123-766A-17R-5, 29-32 cm, $\times 60$. 

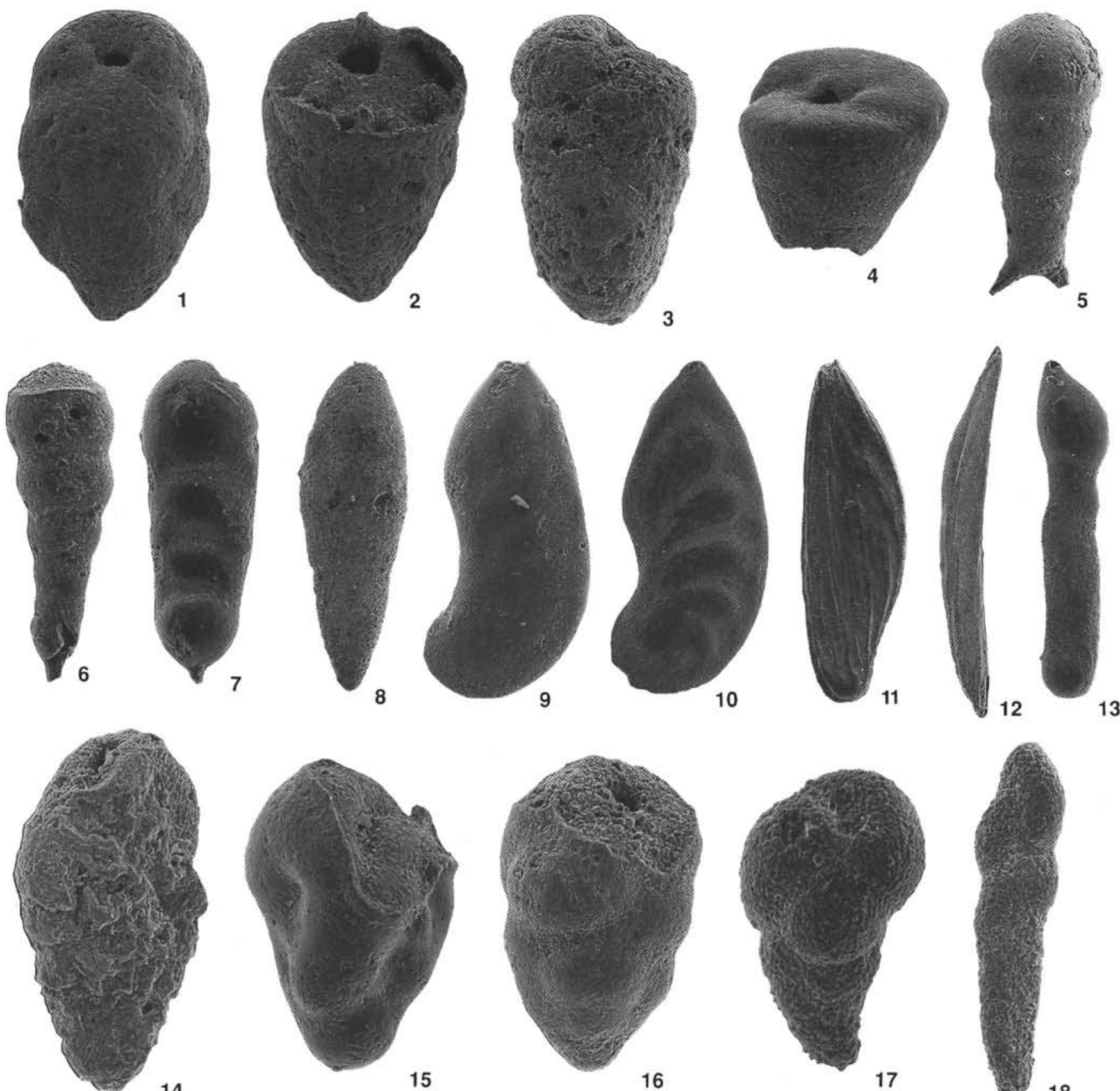

14

15

16

17

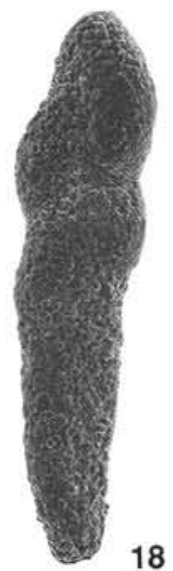

Plate 2. 1,2. Remesella sp., (1) Sample 123-766A-16R-5, 60-64 cm, $\times 70$, (2) Sample 123-766A-16R-5, 60-64 cm, $\times 75$. 3. Dorothia sp., Sample 123-766A-16R-5, 60-64 cm, $\times 65$. 4. Marssonella oxycona (Reuss), Sample $123-766 \mathrm{~A}-16 \mathrm{R}-5,60-64 \mathrm{~cm}, \times 150.5,6$. Lingulonodosaria sp. B, (5) Sample 123-766A-21R-CC, $\times 145,(6)$ Sample 123-766A-25R-CC, $\times 165$. 7. Lingulonodosaria sp. A, Sample 123-766A-21R-1, 90-94 cm, $\times 135$. 8. Lingulina sp., Sample 123-766A-21R-CC, $\times 150$. 9. Astacolus cf. scitula (Berthelin), Sample $123-766 \mathrm{~A}-20 \mathrm{R}-3,68-72 \mathrm{~cm}, \times 100$. 10. Astacolus sp. A, Sample 123-766A-18R-5, 93-96 cm, $\times 90$. 11. Citharina sp. A, Sample 123-766A-17R-3, 36-39 cm, $\times 60$. 12. Citharina sp. B, Sample 123-766A-21R-CC, $\times 85$. 13. Vaginulina sp., Sample $123-766 \mathrm{~A}-20 \mathrm{R}-3,68-72 \mathrm{~cm}, \times 85$. 14. Tappanina sp., Sample $123-766 \mathrm{~A}-16 \mathrm{R}-3,46-50 \mathrm{~cm}, \times 270.15$. Cuneus cf. ludbrookae (Haig), Sample 123-766A-16R-3, 46-50 cm, $\times 220$. 16. (?)Eurycheilostoma $\mathrm{cf}$. hergottensis (Ludbrook), Sample 123-766A-18R-3, 65-67 cm, $\times 235$. 17. Praebulimina cf. nannina (Tappan), Sample 123-766A-20R-3, 68-72 cm, $\times 225$. 18. Coryphostoma sp., Sample 123-766A-20R-3, 68-72 cm, $\times 130$. 

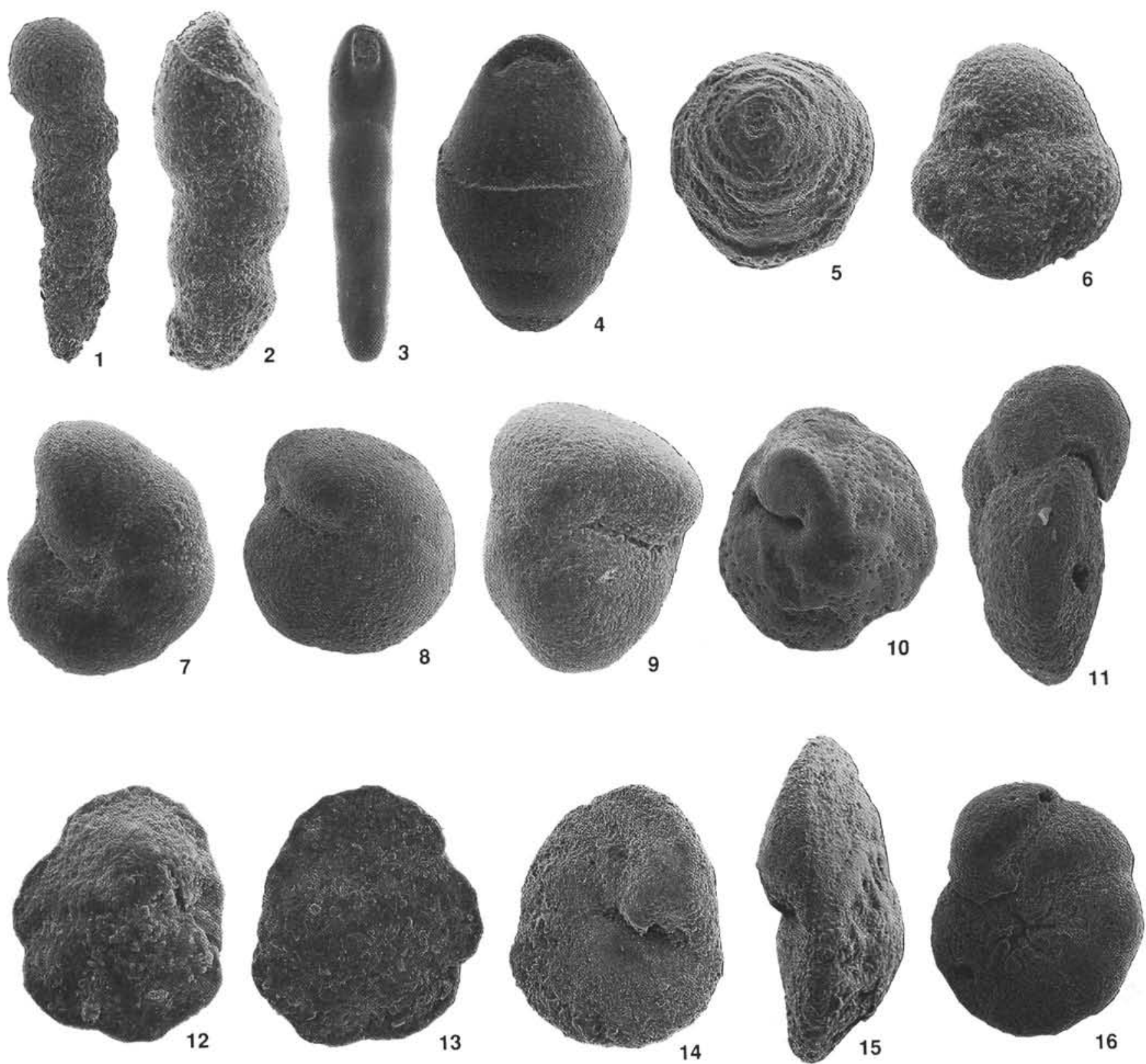

Plate 3. 1. Ellipsoidella $\mathrm{cf}$. cuneata (Loeblich and Tappan), Sample $123-766 \mathrm{~A}-20 \mathrm{R}-\mathrm{CC}, \times 135.2,3$. Pleurostomella aff. reussi Berthelin, (2) Sample 123-766A-20R-3, 68-72 cm, $\times 160$, (3) Sample 123-766A-16R-3, 46-50 cm, $\times 100$. 4. Ellipsoglandulina sp., Sample 123-766A-16R-CC, $\times 100$. 5. Hergottella sp., Sample 123-766A-25R-1, 33-35 cm, spiral view, $\times 160$. 6. Quadrimorphina allomorphinoides (Reuss), Sample 123-766A-17R-CC, umbilical view, $\times 160$. 7. Serovaina gracillima (ten Dam), Sample 123-766A-17R-CC, umbilical view, $\times 135.8,9$. Serovaina infracretacea (Morozova), Sample 123-766A-20R-3, 68-72 cm, (8) umbilical view, $\times 100,(9)$ peripheral view, $\times 125.10$. Globorotalites aptiensis Bettenstaedt, Sample 123-766A-16R-5, 60-64 cm, umbilical view, $\times 90.11,16$. Berthelina intermedia (Berthelin), Sample 123-766A-20R-3, 68-72 cm, (11) peripheral view, $\times 135,(12)$ umbilical (oral) view, $\times 110,12,13$. Charltonina $\mathrm{cf}$, australis Scheibnerová, Sample 123-766A-17R-CC, (12) umbilical view, $\times 160$, (13) spiral view, $\times 160$. 14,15. Osangularia schloenbachi (Reuss), Sample 123-766A-20R-3, 68-72 cm, (14) umbilical view, $\times 125,(15)$ peripheral view, $\times 150$. 

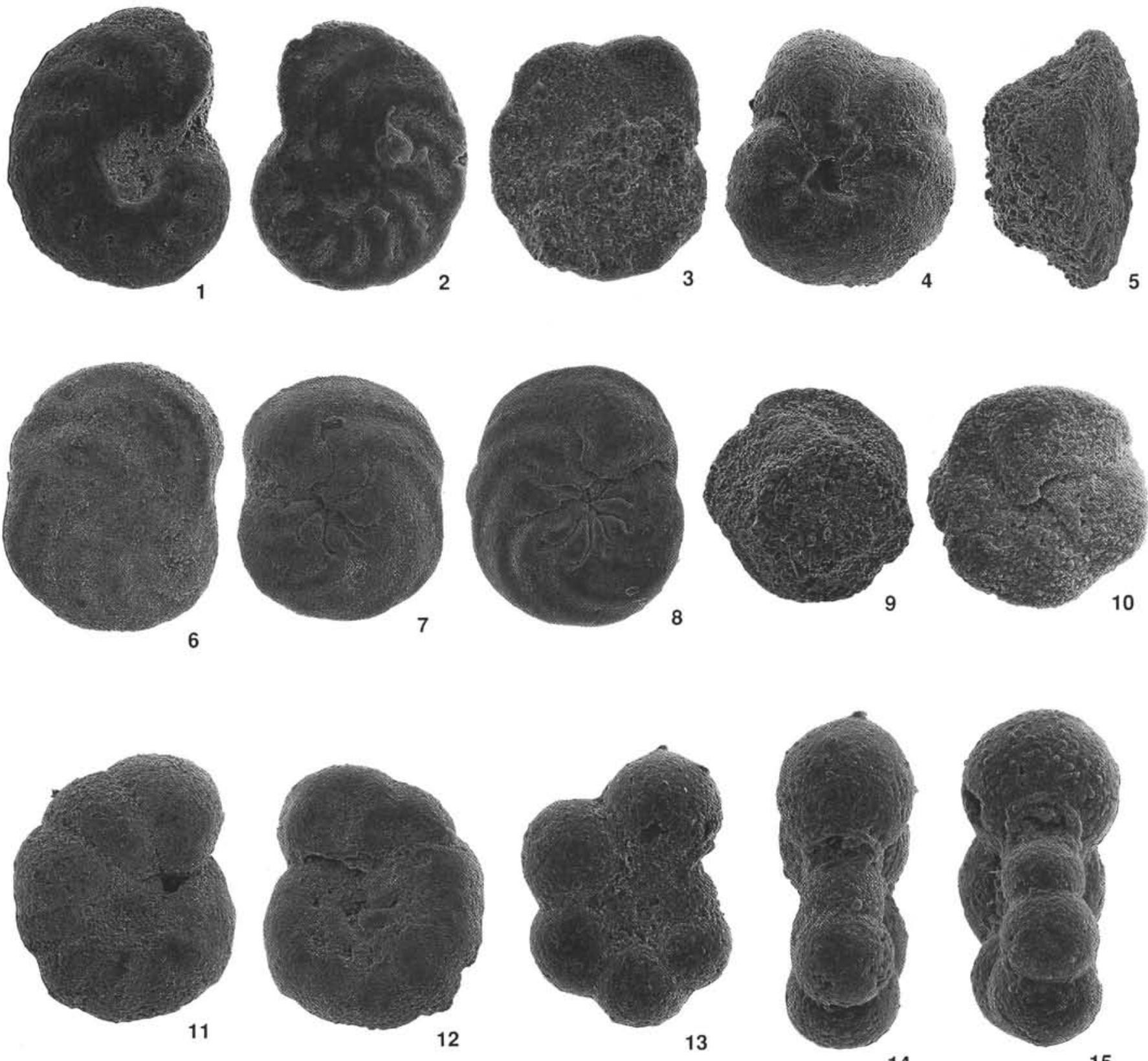

Plate 4. 1,2. Gavelinella cf. andersoni (Church), Sample 123-766A-18R-5, 93-96 cm, (1) umbilical view, $\times 180,(2)$ spiral view, $\times 175.3,4$. Gavelinella cf. indica (Scheibnerová), Sample 123-766A-20R-3, 68-72 cm, (3) spiral view, $\times 130$, (4) umbilical view, $\times 130.5,9,10$. Scheibnerova protindica Quilty, Sample 123-766A-17R-CC, (5) peripheral view, $\times 165$, (9) spiral view, $\times 140$, (10) umbilical view, $\times 150.6,7,8$. Gavelinella sp. A, $(6,7)$ Sample 123-766A-20R-3, 68-72 cm, (6) spiral view, $\times 70$, (7) umbilical view, $\times 65$, (8) Sample 123-766A-20R-3, 68-72 cm, umbilical view, $\times 70$. 11,12. Gavelinella sp. B, Sample 123-766A-21R-2, 52-56 cm, (11) spiral view, $\times 70,(12)$ umbilical view, $\times 70.13,14,15$. Globigerinelloides aptiense Longoria, $(13,14)$ Sample 123-766A-25R-CC, (13) lateral view, $\times 250$, (14) peripheral view, $\times 300,(15)$ Sample $123-766 \mathrm{~A}-25 \mathrm{R}-\mathrm{CC}$, peripheral view, $\times 375$. 

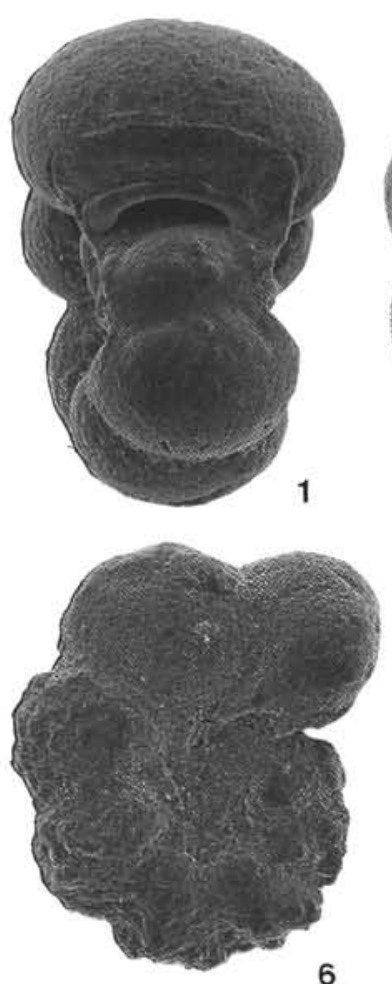

6

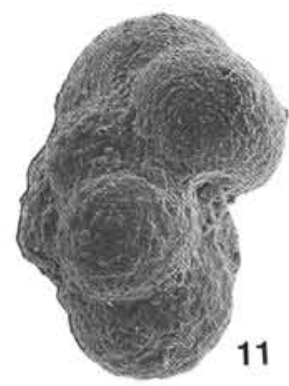

11

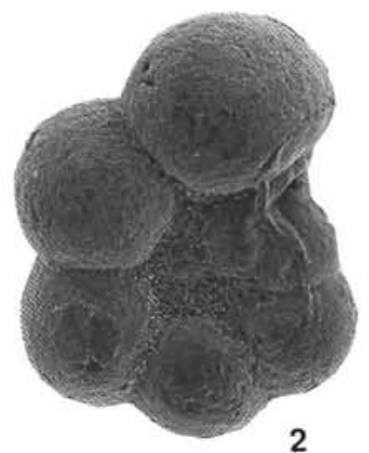

2
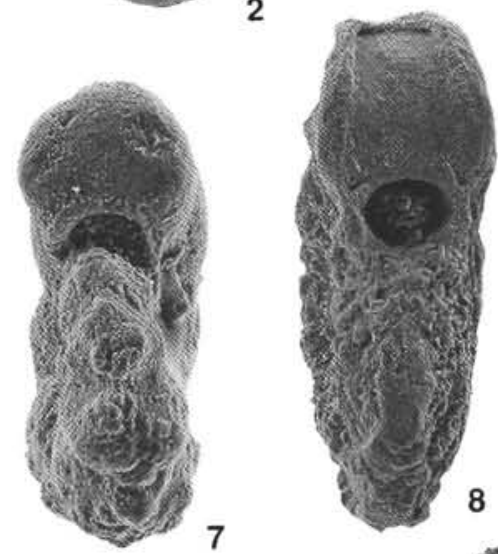

7

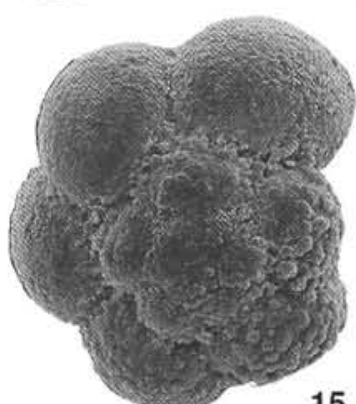

15

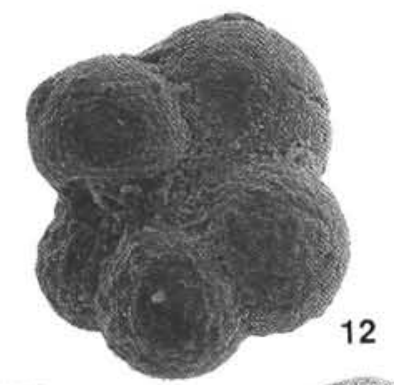

12

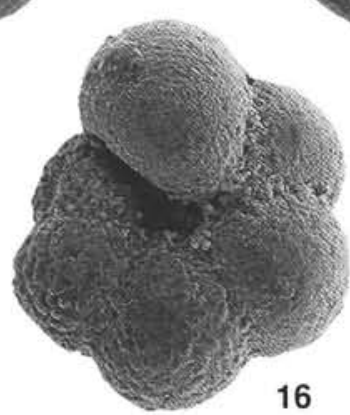

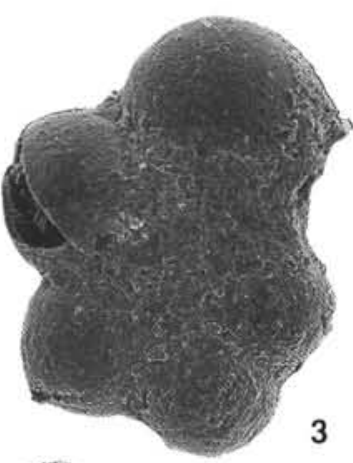

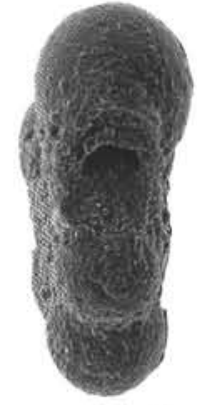

4
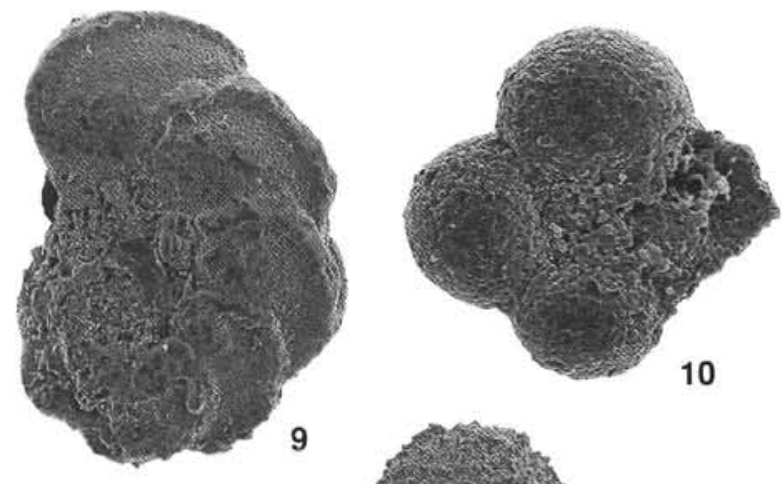

10

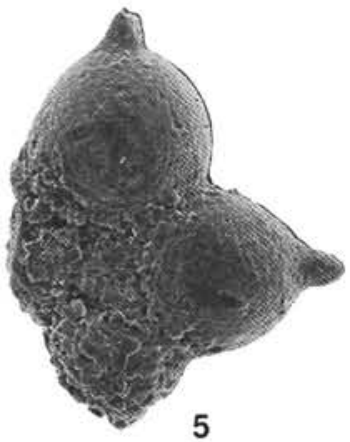

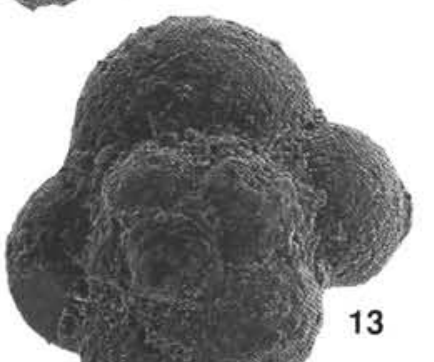
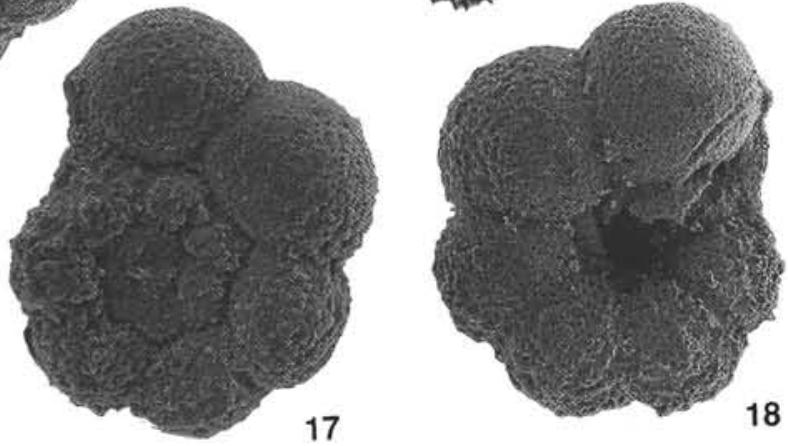

Plate 5. 1,2. Globigerinelloides cf. bentonensis (Morrow), Sample 123-766A-24R-CC, (1) peripheral view, $\times 150$, (2) lateral view, $\times 160$. 3,4. Globigerinelloides $\mathrm{cf}$. aptiense Longoria, Sample 123-766A-25R-CC, (3) lateral view, $\times 225,(4)$ peripheral view, $\times 205$. 5. Schackoina cenomana (Schacko), Sample 123-766A-16R-3, 46-50 cm, $\times 280.6,7$. Planomalina praebuxtorfi Wonders, Sample 123-766A-17R-2, 125-128 cm, (6) lateral view, $\times 205$, (7) peripheral view, $\times 215$. 8,9. Planomalina buxtorfi (Gandolfi), Sample 123-766A-16R-3, $46-50 \mathrm{~cm},(8)$ peripheral view, $\times 110,(9)$ lateral view, $\times 100$. 10. Clavihedbergella sp., Sample 123-766A-19R-CC, $\times 305.11-13$. Hedbergella cf. Blefuscuiana aptica (Agalarova), Sample 123-766A-25R-CC, (11) peripheral view, $\times 225$, (12) umbilical view, $\times 210$, (13) spiral view, $\times 210$. 14. Clavihedbergella simplicissima (Magné and Sigal), Sample 123-766A-16R-CC, umbilical view, $\times 135,15,16$. Hedbergella cf. planispira (Tappan), Sample 123-766A-24R-CC, (15) spiral view, $\times 220$, (16) umbilical view, $\times 210.17,18$. Hedbergella planispira (Tappan), Sample 123-766A-19R-CC, (17) spiral view, $\times 175,(18)$ umbilical view, $\times 175$. 

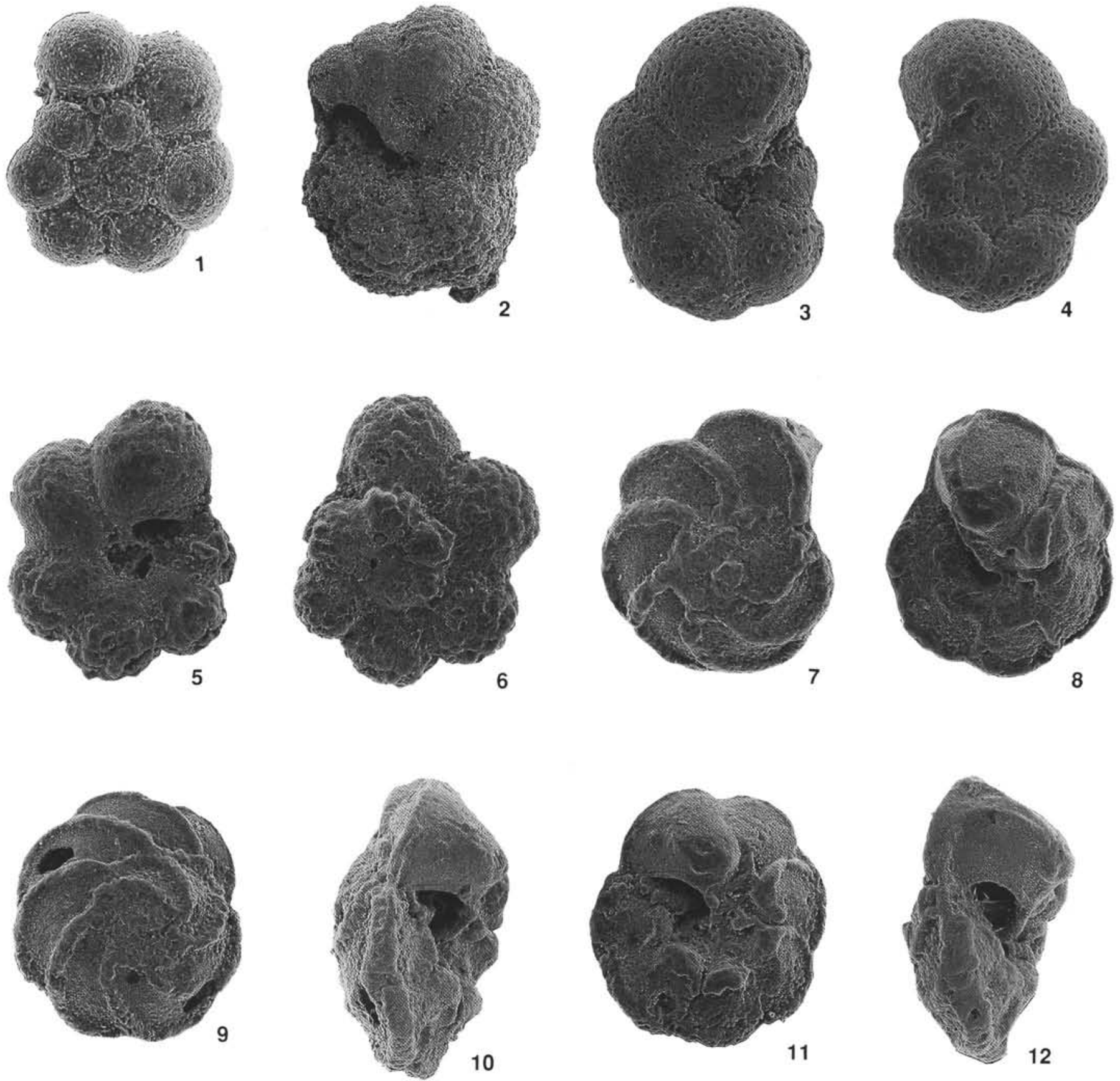

Plate 6. 1. Hedbergella $\mathrm{cf}$. punctata Michael, Sample 123-766A-17R-CC, spiral view, $\times 255$. 2. Hedbergella trocoidea (Gandolfi), Sample 123-766A$21 \mathrm{R}-\mathrm{CC}$, umbilical view, $\times 100.3,4$. Hedbergella yezoana Takayanagi and Iwamoto, Sample $123-766 \mathrm{~A}-16 \mathrm{R}-3,46-50 \mathrm{~cm}$, (3) umbilical view, $\times 170$, (4) spiral view, $\times 160$. 5,6. Praeglobotruncana delrioensis (Plummer), Sample 123-766A-16R-3, 46-50 cm, (5) umbilical view, $\times 135,(6)$ spiral view, $\times 135.7,8,12$. Rotalipora appenninica (Renz), Sample 123-766A-16R-3, 46-50 cm, (7) spiral view, $\times 65,(8)$ umbilical view, $\times 60,(12)$ peripheral view, $\times 65.9,10,11$. Rotalipora ticinensis (Gandolfi), Sample 123-766A-16R-3, 46-50 cm, (9) spiral view, $\times 85,(10)$ peripheral view, $\times 105,(11)$ umbilical view, $\times 90$. 